On the number of the SRB measures for surface endomorphisms

Pouya Mehdipour Balagafsheh 


\title{
On the number of the SRB measures for surface endomorphisms
}

\author{
Pouya Mehdipour Balagafsheh
}

Orientador: Prof. Dr. Ali Tahzibi

Doctoral dissertation submitted to the Instituto de Ciências Matemáticas e de Computação ICMC-USP, in partial fulfillment of the requirements for the degree of the Doctorate Program in Mathematics FINAL VERSION 


\title{
Sobre números das medidas SRB para endomorfismos da superfície.
}

\author{
Pouya Mehdipour Balagafsheh
}

Orientador: Prof. Dr. Ali Tahzibi

Tese apresentada ao Instituto de Ciências Matemáticas e de Computação - ICMC-USP, como parte dos requisitos para obtenção do título de Doutor em Ciências - Matemática VERSÃO REVISADA . 
Ficha catalográfica elaborada pela Biblioteca Prof. Achille Bassi e Seção Técnica de Informática, ICMC/USP, com os dados fornecidos pelo(a) autor(a)

\begin{tabular}{|c|c|}
\hline \multirow[t]{3}{*}{ M4980 } & $\begin{array}{l}\text { Mehdipour Balagafsheh, Pouya } \\
\text { On the Number of the SRB measures for surface } \\
\text { endomorphisms / Pouya Mehdipour Balagafsheh; } \\
\text { orientador Ali Tahzibi. -- São Carlos, } 2014 \text {. } \\
\quad 69 \text { p. }\end{array}$ \\
\hline & $\begin{array}{l}\text { Tese (Doutorado - Programa de Pós-Graduação em } \\
\text { Matemática) -- Instituto de Ciências Matemáticas e } \\
\text { de Computação, Universidade de São Paulo, } 2014 \text {. }\end{array}$ \\
\hline & $\begin{array}{l}\text { 1. Sistemas dinâmicos. 2. Teoria ergódica. } 3 . \\
\text { Teoria de Pesin sobre dinâmico não uniformamente } \\
\text { hiperbolico. 4. Medidas SRB. I. Tahzibi, Ali, } \\
\text { orient. II. Título. }\end{array}$ \\
\hline
\end{tabular}


$* * *$

To Shahab, my gone friend, in the name of his entire eagerness for science And to Mostafa, for his patience and all the glad memories, we had together here in Brazil. 


\section{Acknowledgment}

I would like to express my deep gratitude to my Supervisor, Prof. Dr. Ali Tahzibi, for his guidance, care, patience, and accepting me as a $\mathrm{PhD}$ candidate. "Dear Dear Dr Ali, you were not only a great advisor that a real respectable friend whom I will never forget in my life".

Also i would like to precise my best thanks to Prof Dr. Maria Aparecida Ruas (Cidinha) and Dr Marcia C.A.B. Federson for their kind supports and grateful personality. Many thanks to Prof. Carlos A. Maquera and Prof Daniel Smania thanks for maintaining a friendly atmosphere for the dynamical system group of ICMC and i enounce my humble appreciation to Dear Prof. Farid and Dear Sofia for their kind kind friendships.

My regards to Brazillian Coordination of Improvement of Higher Education Personnel (CAPES) for supporting my Ph.D studies in Brazil and ICMC/USP for its healthy and sincere environment to do research and studies.

Discovering the new worlds like deciding new paths for your life, is a challenging adventure in the beginning. You learn a lot and visit the ones, with bold footprints in the story of your life. New friendships are always the most precious gifts in my life. During my first travel to Brasil i remember asking myself, if it is possible to make true friendships all over the world either being outside your own country, and the answer was YES! I am prideful of my friendships made in Brasil with friends from different nationalities. Thankful of God, blessing me valuable gifts like Pedro. Lima, Jaqueline Godoy, Renato Alejandro, Nazira Hanna Harb, Patricia Hilario Tacuri, Norbil, Iris, Amanda, Jose, Nancy, Jessyca, Almas, Iram, Sarosh, Ebrahim, Mostafa, Alireza, Haniyeh, Abasalt, Fatemeh, Dr Behruz, Dr Parham, Dear Salimeh and all my other colleagues although i am not mentioning all the names here.

My academic brothers Thiago, Marcos, Fernando, Gabriel Mantovani, Jose Santana, 
Jorge, and specially my youngest precious Brother Gabriel Ponce whom i learnt a lot and i will never forget the good memories we had in the math way together. My Grand brothers Pablo, Regis and Fernando meeting you guys was a nice Honor in my life and I feel proudness of having you good friends in my academic career.

And at the End i would Like to put my best privileged thanks to beloved Parents, Mrs Tahzibi, Dear Maria, Parents of Mostafa, Parents of Almas and Iram, Parents of Gabriel, Parents of Pedro, My merciful Mom and lovely Dad, My Dearest sisters Mona, Poorna also my cousin Shoja. You were always supporting me and encouraging me with your best wishes and prayers. I hope to have all you happy, healthy and with long life if God wants.

Pouya Mehdipour

Sao Carlos, 2014. 


\section{Abstract}

Let $f$ be a $C^{2}$ local diffeomorphism, of a closed surface $M$ without zero Lyapunov exponents. We have proved that the number of ergodic hyperbolic measures of $f$ with $\mathrm{SRB}$ property is less than equal to the number of homoclinic equivalence classes. We use an adaptation of Katok closing lemma for endomorphisms and prove ergodic criterion, introduced in [HHTU], for endomorphisms.

We also prove some folklore results on uniqueness of SRB measures, in the presence of topological transitivity. 


\section{Resumo}

Seja $f$ um endomorfismo $C^{2}$ non-singular (difeomorfismo local), de uma superfície fechada $M$ e $\mu$ uma medida probabilidade Borel f-invariante e ergódica com expoentes de Lyapunov Não nulo. Nós provamos que o número de medidas hiperbólicas com propriedade SRB é para $f$ so menor ou igual ao número de classes equivalentes homoclínicos. Usamos uma adaptaão do closing lema de Katok por endomorfismos e provamos critrio ergódico, introduzido em [HHTU], para endomorfismos. Também provamos alguns resultados folclóricos em unicidade de medidas SRB, na presena de transitividade topológica. 


\section{Contents}

Acknowledgment $\quad$ iii

$\begin{array}{lll}\text { Abstract } & \text { v }\end{array}$

Abstract $\quad$ vii

Introduction 1

1 Non-uniform Hyperbolicity for Endomorphisms 5

1.1 Inverse Limit Space . . . . . . . . . . . . . . . . . . . . . . . 5

1.2 Multiplicative Ergodic Theorem . . . . . . . . . . . . . . . . . . . . 9

1.2.1 Multiplicative Ergodic Theorem on Natural Extension . . . . . . . 11

1.3 Nonuniform-Hyperbolicity and Pesin Sets . . . . . . . . . . . . . . . . . . 12

1.4 Pesin Stable-Unstable Manifolds . . . . . . . . . . . . . . . . . . . . . . . . 14

1.5 Absolute Continuity and Conditional Measures . . . . . . . . . . . . . . 17

1.5.1 Lambda Lemma . . . . . . . . . . . . . . . . . . . . . . . . . 19

1.6 SRB Property and Main Results . . . . . . . . . . . . . . . . . . . 20

1.6.1 Ergodic Component and SRB Property . . . . . . . . . . . . . . 22

2 Katok Closing Lemma $\quad 25$

2.1 Lyapunov charts . . . . . . . . . . . . . . . . 25

2.2 Admissible Manifolds . . . . . . . . . . . . . . . . . . . . . . . . . . 29 
2.3 Katok closing lemma for Endomorphisms . . . . . . . . . . . . . . . . . . 31

2.4 Hyperbolic Periodic Orbits and Horseshoe . . . . . . . . . . . . . 38

3 Ergodic Homoclinic Classes and Proof of Theorems 41

3.1 Ergodic Homoclinic Classes. . . . . . . . . . . . . . . . . . . . . . . 41

3.2 Ergodic Criterion. . . . . . . . . . . . . . . . . . . . 44

3.3 Proof of Theorems . . . . . . . . . . . . . . . . . . . . . 49

3.3.1 Proof of the Main Theorem . . . . . . . . . . . . . . . . . 50

3.3.2 Proof of Theorem B . . . . . . . . . . . . . . 52

3.3.3 Proof of Theorem C . . . . . . . . . . . . . . 53

4 Examples and Problems $\quad 55$

4.1 Example I . . . . . . . . . . . . . . . . . . . . . 56

4.2 Example II . . . . . . . . . . . . . . . . . . . . 57

4.3 Example III . . . . . . . . . . . . . . . . . . . . . . . . . 59

4.4 Example IV . . . . . . . . . . . . . . . . . . . . . . 61

$\begin{array}{lll}5 & \text { Appendix A } & 63\end{array}$

$\begin{array}{ll}\text { Bibliography } & 67\end{array}$ 


\section{Introduction}

According to the history of science, the birth of "Chaos" goes to H.Poincaré and the king of Sweden's question on the stability of the solar system around 1889. However after that, for at least seven decades we may observe no specific intention on chaotic dynamics, but after the mid of twentieth-century when E. Lorenz put forward his work on weather forecasting and give birth to attractors, determination of chaotic dynamics became one of the most remarkable attempts. In fact, it is observed that even very simple systems depending only on one variable, may behave as wild as a hurricane or a volcano performing chaotic behavior. "Changes in weather", "the planet orbits in the solar system",[M2], "population growth in ecology", "the dynamics of the action potentials in neurons" [RY], "molecular vibrations" and a lot more, all are samples in nature not predictable but depending to the initial conditions, deterministic.

Imagine a closed (compact connected boundaryless) Riemannian manifold with a uniformly "hyperbolic" dynamics. The tangent space at every point decomposes in two complementary subspaces, one expanding and the other contracting along the trajectory. It is obvious that for such phase space, in the lack of enough space trajectories can not travel separately and mixing together exhibit strange or chaotic behavior. The weakest but most general type of hyperbolicity is the so called "Non- Uniform Hyperbolicity" introduced by Y. Pesin in 1970. However depending on the initial condition, it may happen wild uncontrollable trajectories, but almost all orbits of the system show a kind of tameness like living in a uniformly hyperbolic world.[BP1]

Looking at the both, natural and artificial physical models, not all of them have the property that their dynamics is invertible. The study of non-invertible (endomorphism) maps nowadays have its own importance. By definition an endomorphism is a continuouse map which is not necessarily invertible. In this work in special we deal with non- singular endomorphisms or local diffeomorphism where always exist some small ball around each 
point of the space, diffeomorphically projecting on its image. Getting some idea about the differences between invertible and non-invertible maps, we can look at the unstable manifolds. They are one of the main objects related to chaos theory and depending upon the past history of an orbit, not unique for endomorphisms. To approach chaotic properties of an endomorphism, typically we define some inverse limit space. It is the collection of the all possible total orbits in a phase space $M$. Although this space $M^{f}$, does not admit a manifold structure but it is a compact metric sub-space of $M^{\mathbb{Z}}$ and many mathematical notions depending on pre-histores can be carried on it. In 1960s Shub [Sh] introduced expanding maps and after that Mané-Pugh[] and Przytycki[P] found other properties for Anosov endomorphisms (with uniform hyperbolicity). A remarkable result shows that Anosov endomorphisms are not structurally stable.

In smooth ergodic theory, we study the ergodic properties of smooth dynamical systems on Riemannian manifold up to an invariant measure, specially one equivalent to Riemannian volume. In this theory which provides a mathematical basis for the theory of deterministic chaos, the two main tools are "Lyapunov Exponents" and "Metric Entropy". First implement measures the asymptotic exponential behavior along the long term orbit and the second, measures the complexity of the dynamics under the iterations.

In 1970s Sinai, Ruelle and Bowen introduced an invariant measure which is called SRB measure. Their original work was on Axiom A and Anosov dynamical systems (uniform hyperbolicity along all the orbits). These measures play an important role in the ergodic theory of dissipative dynamical systems (not preserving the volume) and are the most compatible measures in the occasion.

J.-M. Strelcyn, F. Ledrappier and L-S.Young in [LS] and [LY] have shown that for $f$ a $C^{2}$ diffeomorphism on a compact Riemannian manifold, and for $\mu$ an $f$-invariant Borel probability measure, metric entropy is equal $\mu$-a.e. to the sum of positive Lyapunov exponent. Moreover this so called "Pesin entropy formula" holds if and only if $\mu$ is an SRB measure. These results were generalized to other frameworks including deterministic endomorphisms by the works of Qian, Xie and Zhu [QXZ]. New results can be still scattered in this frame of studies.

In recent years F. Rodriguez Hertz, M. A. Rodriguez Hertz, A. Tahzibi, R. Ures in [HHTU] have shown the uniqueness of SRB measures for transitive $C^{1+\alpha}$ diffeomorphisms on compact surfaces. In this thesis, we investigate whether the same method of [HHTU] works on $C^{2}$ surface endomorphisms or not. A main part of their result can be translated into the endomorphisms setting. Howbeit, there exist many subtle differences between the two cases. For instance, the uniqueness of SRB measures which can be deduced 
using topological transitivity for diffeomorphisms, in our setting it may not be the case. Although we do not have any example of topologically transitive surface endomorphism with more than one SRB measure, a modification of Kan example may provide such new phenomena. ${ }^{1}$

With respect our main theorem, the number of SRB measures for surface endomorphisms(see definition 1.34) is bounded by the number of homoclinic equivalence classes. In other words, we can control the number of SRB measures (Ergodic objects) by a topological substance. More precisely, we prove that:

Theorem 0.1 (Main Theorem A). Let $f: M \rightarrow M$ be a $C^{2}$ endomorphism over a closed Riemannian surface $M$. Then,

$\sharp\{$ Ergodic Hyperbolic Measures with SRB Property $\} \leq \sharp\{$ Homoclinic Equivalent Classes. $\}$

For the definition of homoclinic equivalent class, see Chapter (1). There are some other folkloric and simple results about the uniqueness of measures with SRB property which we prove for the sake of completeness:

Theorem 0.2 (Theorem B). For $f: M \rightarrow M$ a topologically transitive $C^{2}$ endomorphism over a closed Riemannian surface $M$, there exists at most one non-uniformly expanding measure with SRB property.

Theorem 0.3 (Theorem C). Let $f: M \rightarrow M$ be a topologically transitive $C^{2}$ partially hyperbolic endomorphism with a continuous decomposition of the tangent bundle $T M=$ $E^{s} \oplus E^{c u}$. Then there exists at most one hyperbolic measure with SRB property.

The main problems we deal with through this work consist of:

Non-uniform Hyperbolicity for endomorphisms;

Closing lemma of Katok for endomorphisms;

Ergodic Homoclinic Classes and ergodic criterion;

Chapter (1) of this thesis, gives a short survey on the theory of non-uniform hyperbolicity for endomorphisms. It contains some preliminaries on inverse limit space and we bring the "Multiplicative ergodic theorem" of Oseledets on natural extension as well as notions about non-uniform hyperbolicity, Pesin blocks, Pesin stable-unstable sets, absolute continuity, Lambda lemma and SRB property for endomorphisms.

\footnotetext{
${ }^{1}$ We would like to thank M. Andersson and J. Yang for observing us the possibility of the construction of such examples.
} 
Chapter (2) is an adaptation of the Katok closing lemma for $C^{2}$ endomorphisms of a closed Rimannian surface. We define the Lyapunov charts and show that the proof of closing lemma and consequences for endomorphisms.

Chapter (3) presents the definition of ergodic homoclinic classes for new setting and bring the theorems and lemmas related to the ergodic criterion part of the [HHTU] method. After that we prove the main theorem and theorems B and C.

Chapter (4) contains some examples and some problems, which we believe that theorems can be applied. 


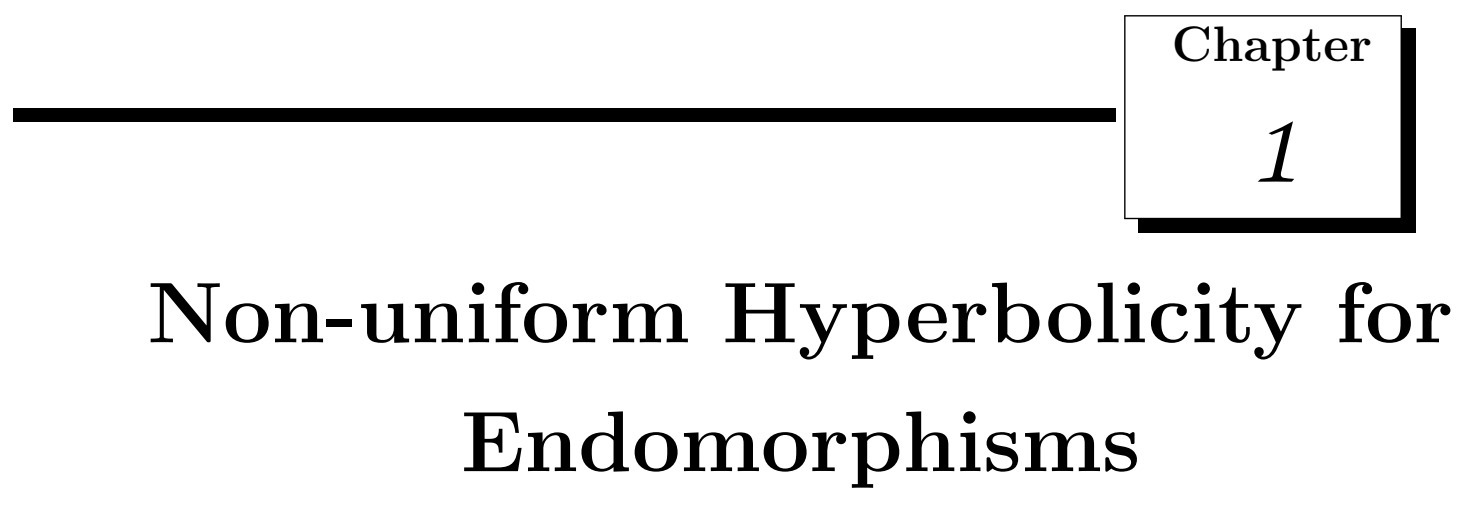

The theory of smooth dynamical systems with non-uniformly hyperbolic behavior, or the theory of non-uniformly hyperbolic dynamical systems is due to Ya.B. Pesin in the mid-1970. In this chapter we try to give a summary of this theory for endomorphisms based on [QXZ] and [BP2].

\subsection{Inverse Limit Space}

Let $M$ be a smooth Riemannian surface which is closed (compact connected and without boundary). In this text by a $C^{2}$ endomorphisms $f: M \rightarrow M$ we mean a local $C^{2}$ diffeomorphism and we denote $\mathcal{M}_{f}(M)$ for the set of all $f$-invariant Borel probability measures. As $f$ is a local diffeomorphism, it always satisfies the following integrability condition and we may omit it.

$$
\log \left|\operatorname{det} d_{x} f\right| \in \mathcal{L}^{1}(M, \mu)
$$

For $f: M \rightarrow M$ a $C^{2}$ endomorphism, consider the compact metric space

$$
M^{f}=\left\{\tilde{x}=\left(x_{n}\right) \in \prod_{-\infty}^{\infty} M: f\left(x_{n}\right)=x_{n+1}, \text { for all } n \in \mathbb{Z}\right\}
$$

equipped with the distance $\tilde{d}$ for $\tilde{x}=\left(x_{n}\right), \tilde{y}=\left(y_{n}\right) \in M^{f}$ defined by 


$$
\tilde{d}(\tilde{x}, \tilde{y})=\sum_{n=-\infty}^{\infty} 2^{-|n|} d\left(x_{n}, y_{n}\right) .
$$

Where $d$ is the distance on $M$ induced by the Riemannian metric. Let $\pi$ be the natural projection from $M^{f}$ to $M$ (i.e. $\pi\left(\left(x_{n}\right)\right)=x_{0}, \forall \tilde{x}=\left(x_{n}\right) \in M^{f}$,) and $\tilde{f}: M^{f} \rightarrow M^{f}$ be the shift homeomorphism. We may sometimes call $x_{0}$ as the base point of a trajectory. It is clear that the following diagram commutes i.e. $\pi \circ \tilde{f}=f \circ \pi$.

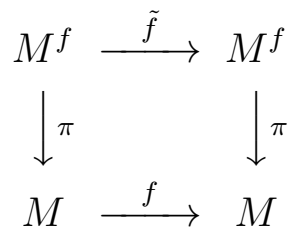

The map $\tilde{f}: M^{f} \rightarrow M^{f}$ is called the inverse limit of $f$ or the natural extension of system $(M, f)$ and the proper $M^{f}$ is the Inverse Limit Space. Notice that for a periodic point $p$ of $f$ with period $m$, we may denote the unique corresponded periodic point on $M^{f}$, with $\bar{p}=\left(\ldots p f^{m-1}(p) \ldots f(p) p, \ldots\right) \in M^{f}$.

The map $\pi$ induces a continuous map from $\mathcal{M}_{\tilde{f}}\left(M^{f}\right)$ to $\mathcal{M}_{f}(M)$, usually denoted by $\pi_{*}$ i.e. for any $\tilde{f}$-invariant Borel probability measures $\tilde{\mu}$ on $M^{f}, \pi_{*}$ maps it to a $f$-invariant Borel probability measure $\pi_{*} \tilde{\mu}$ on $M$ defined as

$$
\pi_{*} \tilde{\mu}(\phi)=\tilde{\mu}(\phi \circ \pi), \quad \forall \phi \in C(M)
$$

The following proposition I.3.1 of [QXZ] or [OV] guarantees that $\pi_{*}$ is a bijection between $\mathcal{M}_{\tilde{f}}\left(M^{f}\right)$ and $\mathcal{M}_{f}(M)$.

Proposition 1.1. Let $f$ be a continuous map on $M$. For any $f$-invariant Borel probability measure $\mu$ on $M$, there exists a unique $\tilde{f}$-invariant Borel probability measure $\tilde{\mu}$ on $M^{f}$ such that $\pi_{*} \tilde{\mu}=\mu$.

Proof. Proof of this theorem is based on the next lemma.[QXZ]

Lemma 1.2. Let $X$ and $Y$ be two compact metric spaces, and $T: X \rightarrow X$ and $S: Y \rightarrow Y$ measurable mappings on corresponding spaces. Suppose there is a continuous surjective map $h: X \rightarrow Y$ such that $S \circ h=h \circ T$. Then for any $S$-invariant Borel probability measure $\mu$ on $Y$, there is a $T$-invariant Borel probability measure $\nu$ on $X$ such that $h \nu=\mu$.

Let us continue the proof of the proposition: 
As a consequence of Lemma 1.2 , there is $\tilde{\mu} \in \mathcal{M}_{\tilde{f}}\left(M^{f}\right)$ such that $\pi_{*}(\tilde{\mu})=\mu$. Since $M^{f}$ is a compact subset of $M^{\mathbb{Z}}$ defined as a shift space, so cylinders make a second countable basis for it. Then $\tilde{\mu}$ can be uniquely determined by its values on all cylinder sets. For any subsets $A_{0}, A_{1}, . ., A_{n} \subset M$ we define

$$
\tilde{\mu}\left(\left[A_{0}, A_{1}, . ., A_{n}\right]\right)=\mu\left(A_{0} \cap f^{-1} A_{1} \cap \ldots \cap f^{-n} A_{n}\right),
$$

where

$$
\left[A_{0}, A_{1}, . ., A_{n}\right]=\left\{\tilde{x} \in M^{f} \mid x_{i} \in A_{i}, i=0,1, \ldots, n\right\}
$$

is a cylinder in $M^{f}$. Observe that this definition coincides with $\pi_{*}(\tilde{\mu})=\mu$. Suppose $\left[A_{0}\right]$ be a cylinder in $M^{f}$ then $\pi\left(\left[A_{0}\right]\right)=A_{0}$ and

$$
\pi_{*} \tilde{\mu}\left(A_{0}\right)=\tilde{\mu}\left(\pi^{-1}\left(A_{0}\right)\right)=\tilde{\mu}\left(\left[A_{0}\right]\right)=\mu\left(A_{0}\right) .
$$

Also taking any open $B \subset M$, then $\pi_{*} \tilde{\mu}(B)=\tilde{\mu}\left(\pi^{-1}(B)\right)=\tilde{\mu}([B])=\mu(B)$ This ensures that $\tilde{\mu}$ is uniquely determined by $\mu$ and the proof is completed.

A circumstance of proposition 1.1 can be the following lemma.

Lemma 1.3. An $f$-invariant Borel probability measure $\mu$ is ergodic if and only if $\tilde{\mu}$ is ergodic.

Recalling following theorem( [KH])

Theorem 1.4 (Ergodic Decomposition). Let $\mu \in \mathcal{M}_{f}(X)$ and $(X, \mu, \mathcal{B})$ a Borel probability space. Let $\left\{\mu_{x}\right\}_{x \in X}$ be the conditional probability measures with respect to $\mathcal{F}:=\left\{E \subset \mathcal{B} \mid f^{-1} E=E\right\}$. Then

- $\mu=\int_{X} \mu_{x} d \mu(x)$;

- $\mu_{x}$ is invariant for $\mu$-a.e. $x \in X$;

- $\mu_{x}$ is ergodic for $\mu$-a.e. $x \in X$

Let us continue the proof of lemma.

Proof. We have the uniqueness of $\tilde{\mu}$ from last proposition, then consider the following 
diagram which permutes $\tilde{f}$ and $f$.

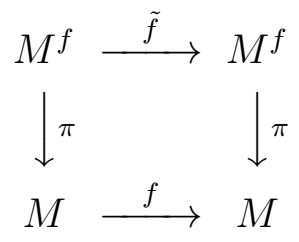

once $\tilde{\mu}$ is ergodic then for each $f$-invariant subset $A \subset M$, we can easily observe that $\pi^{-1}(A)$ is $\tilde{f}$ - invariant and by ergodicity of $\tilde{\mu}$ then $\tilde{\mu}\left(\pi^{-1}(A)\right)=\pi_{*} \tilde{\mu}=\mu(A)$ is zero or one. To show the inverse process let by contradiction suppose that $\mu$ is ergodic but $\tilde{\mu}$ is not ergodic. $M^{f}$ is a compact metric space and $\tilde{\mu} \in \mathcal{M}_{\tilde{f}}\left(M^{f}\right)$. By theorem 1.4 taking $\tilde{\mathcal{F}}=\left\{\tilde{E} \subset \tilde{\mathcal{B}} \mid \tilde{f}^{-1}(\tilde{E})=\tilde{E}\right\}$ and write

$$
\tilde{\mu}=\int_{M^{f}} \tilde{\mu}_{x} d \tilde{\mu}(\tilde{x})
$$

where $\tilde{\mu}_{x}$ is $\tilde{f}$-invariant and ergodic for $\tilde{\mu}$-a.e. $\tilde{x} \in M^{f}$. By Proposition 1.1, the $\tilde{\mu}$ is unique $\tilde{f}$-invariant measure that $\pi_{*} \tilde{\mu}=\mu$. Means

$$
\mu=\pi_{*}(\tilde{\mu})=\int_{M^{f}} \pi_{*} \tilde{\mu}_{x} d \tilde{\mu}(\tilde{x})
$$

The projection of conditional probabilities $\tilde{\mu}_{\tilde{x}}$ either will be $\mu$ or gives some conditional probabilities on $\mathcal{F}=\pi(\tilde{\mathcal{F}})$. In both situation it makes contradiction (respectively) with proposition 1.1 or ergodicity of $\mu$.

Let $M$ be a compact metrizable space, $f: M \rightarrow M$ a continuous map and $\mu$ an $f$-invariant Borel probability measure on $M$. For any finite partition $\eta=\left\{C_{i}\right\}$ of $M$, define the entropy of $\eta$ by

$$
H_{\mu}(\eta)=-\sum_{i} \mu\left(C_{i}\right) \log \mu\left(C_{i}\right)
$$

Let

$$
h_{\mu}(f, \eta)=\lim _{n \rightarrow \infty} \frac{1}{n} H_{\mu}\left(\eta \vee f^{-1} \eta \vee \ldots \vee f^{-n+1} \eta\right) .
$$

Then define the metric entropy of $f$ with respect to $\mu$ as

$$
h_{\mu}(f)=\sup \left\{h_{\mu}(f, \eta): \eta \text { is a finite partition of } M\right\} \text {. }
$$


More information can be found in [W]. From the following proposition we have the relation between the metric entropy of the two systems, $(f, \mu)$ and $(\tilde{f}, \tilde{\mu})$.[QXZ]

Proposition 1.5. Let $f: M \rightarrow M$ be a continuous map on the compact Riemannian manifold $M$ with an invariant Borel probability measure $\mu$. Let $M^{f}$ be the inverse limit space of $(M, f), \tilde{f}$ the shift homeomorphism and $\tilde{\mu}$ the $\tilde{f}$-invariant Borel probability measure on $M^{f}$ such that $\pi_{*} \tilde{\mu}=\mu$. then

$$
h_{\mu}(f)=h_{\tilde{\mu}}(\tilde{f})
$$

\subsection{Multiplicative Ergodic Theorem}

For $\Lambda \subset M$ we set $\Lambda^{f}=\left\{\tilde{x}=\left(x_{n}\right) \in M^{f}: x_{n} \in \Lambda\right.$, for alln $\left.\in \mathbb{Z}\right\}$. Before stating the Oseledet's Multiplicative ergodic theorem, we need to know the definition of a hyperbolic set for endomorphisms.

Definition 1.6 (Hyperbolic Endomorphism-[P]). Let $f$ be a $C^{2}$ endomorphism. Put $\Lambda$ to be an $f$-invariant closed subset of $M$. Then $\Lambda$ is called a Hyperbolic Set for this endomorphism if there exists real constants $C>0$ and $0<\mu<1$ (and a continuous Riemannian metric $<., .>$ on TM) such that for every $f$-trajectory $\tilde{x}=\left(x_{n}\right)_{-\infty}^{+\infty}$ of points in $\Lambda\left(\forall \tilde{x} \in \Lambda^{f}\right)$ and for every integer $n$ we have:

- $T_{x_{n}} M=E^{s}(\tilde{x}, n) \oplus E^{u}(\tilde{x}, n)$,

- $D f\left(E^{s}(\tilde{x}, n)\right)=E^{s}(\tilde{f}(\tilde{x}), n)=E^{s}(\tilde{x}, n+1)$, $\left\|D f_{x_{n}}^{m}(v)\right\| \leq C \mu^{m}\|v\|$, for $v \in E_{x_{n}}^{s}$,

- $\left.D f\left(E^{u}(\tilde{x}, n)\right)=E^{u}(\tilde{f}(\tilde{x}), n)=E^{u}(\tilde{(} x), n+1\right)$, $\left\|D f_{x_{n}}^{m}(v)\right\| \geq\left[C \mu^{m}\right]^{-1}\|v\|$, for $v \in E_{x_{n}}^{u}$.

For $m=0,1, \ldots$ where $\|\cdot\|$ denotes the Riemannian metric. This family of splitting will be called hyperbolic.

We may define the hyperbolicity in term of the cone fields also [HG]. Suppose $\|$. denotes the Riemannian metric on $M$ as above and $\operatorname{End}(M)$ be the set of all regular endomorphisms on $M$ equipped with the usual $C^{1}$ topology.

Let $E$ and $F$ be two sub-bundles of $T M$ satisfying $T M=E \oplus F$. Define two cone fields $C_{\alpha}^{E}$ and $C_{\alpha}^{F}$ on $T M$ : 


$$
\begin{aligned}
& C_{\alpha}^{E}(z)=\left\{v \in T_{z} M: v=\left(v^{E}, v^{F}\right),\left\|v^{F}\right\|<\alpha\left\|v^{E}\right\|\right\} \\
& C_{\alpha}^{F}(z)=\left\{v \in T_{z} M: v=\left(v^{F}, v^{E}\right),\left\|v^{E}\right\|<\alpha\left\|v^{F}\right\|\right\}
\end{aligned}
$$

Definition 1.7. An $f$-invariant subset $\Lambda$ of $M$ is said to be hyperbolic if $T_{\Lambda} M$ has the bundle splitting $T_{\Lambda} M=E \oplus F$ and there is $\alpha>\alpha^{\prime}>0$ and $\lambda>1$ such that, for any $x \in \Lambda$ and any unit vector $v \in C_{\alpha}^{F}(x)$

$$
d f\left(C_{\alpha}^{F}(x)\right) \subset C_{\dot{\alpha}}^{F}(f(x)), \quad\|d f(v)\|>\lambda,
$$

and such that for any $x \in \Lambda$, any unit vector $v \in C_{\alpha}^{E}(f(x))$, and the diffeomorphism $g$ on a neighborhood of $f(x)$ satisfying $g f=i d_{x}$ on a neighborhood of $x$,

$$
d g\left(C_{\alpha}^{E}(f(x))\right) \subset C_{\dot{\alpha}}^{E}(x), \quad\|d g(v)\|>\lambda .
$$

We say that $f$ is an Anosov Endomorphism if $f$ is hyperbolic on $M$. Also $f$ is said to be an Expanding Map if it is an Anosov map without contracting direction $\left(E^{s}=0\right)$.

For $x \in M$ and $f: M \rightarrow M$ suppose $v \in T_{x} M$. We put

$$
\lambda(x, v)=\varlimsup_{n \rightarrow \infty} \frac{1}{n} \log \left\|d_{x} f^{n}(v)\right\| .
$$

The number $\lambda(x, v)$ is called the Lyapunov Exponent for $v$. For $x \in M$ there are at most $(\operatorname{dim} M)$-numbers $\lambda_{1}(x), \cdots, \lambda_{r(x)}(x)$ with $-\infty<\lambda_{1}(x)<\cdots<\lambda_{r(x)}<\infty$, and a filtration of subspaces

$$
\{0\}=L_{0}(x) \subsetneq L_{1}(x) \subsetneq \cdots \subsetneq L_{r(x)}(x)=T_{x} M
$$

(Where $L_{i}(x)=\left\{v \in T_{x} M: \lambda(x, v) \leq \lambda_{i}(x)\right.$, for $\left.1 \leq i \leq r(x)\right\}$.)

and $\lambda(x, v)=\lambda_{i}(x)$ holds for $v \in L_{i}(x) \backslash L_{i-1}(x), 1 \leq i \leq r(x)$. The numbers $\lambda_{1}(x)$ $\cdots \lambda_{r(x)}(x)$ are called the Lyapunov Exponents at $x$. We set

$$
m_{i}(x)=\operatorname{dim} L_{i}(x)-\operatorname{dim} L_{i-1}(x)
$$

for $1 \leq i \leq r(x)$ and it is the Multiplicity of Lyapunov exponent $\lambda_{i}(x)$. Observe that $r(x)$, $\lambda_{i}(x), m_{i}(x)$ are measurable $f$-invariant functions with respect to any $f$-invariant Borel probability measure $\mu$ and when $\mu$ is an ergodic measure, then these functions become 
constant almost everywhere and we may show them by $\lambda_{i}^{\mu}, m_{i}^{\mu}, r^{\mu}$. (Such information are obtained by multiplicative ergodic theorem for differentiable maps [W], [Ch].)

\subsubsection{Multiplicative Ergodic Theorem on Natural Extension}

As a consequence of Oseledet's theorem we have the following fundamental results on $M^{f}[\mathrm{QXZ}]$. we may show it by $M E T$.

Theorem 1.8. Let $\mu$ be an $f$-invariant Borel probability measure on $M$. We denote by $\tilde{\mu}$ the $f$-invariant Borel probability measure on $M^{f}$ such that $\pi_{*} \tilde{\mu}=\mu$. Then for $\tilde{\mu}$ almost all $\tilde{x}=\left(x_{n}\right) \in M^{f}$ and $n \in \mathbb{Z}$ the tangent space $T_{x_{n}} M$ splits into a direct sum $T_{x_{n}} M=E_{1}(\tilde{x}, n) \oplus \cdots \oplus E_{r\left(x_{0}\right)}(\tilde{x}, n)$ and exists $-\infty<\lambda_{1}(\tilde{x})<\cdots<\lambda_{r(\tilde{x})}(\tilde{x})<\infty$ and $m_{i}(\tilde{x})(i=0,1, \ldots, r(\tilde{x}))$, Such that:

1. $\operatorname{dim} E_{i}(\tilde{x}, n)=m_{i}(\tilde{x})$;

2. $d_{x_{n}} f\left(E_{i}(\tilde{x}, n)\right)=E_{i}(\tilde{x}, n+1)$, and $\left.d_{x_{n}} f\right|_{E_{i}(\tilde{x}, n)}: E_{i}(\tilde{x}, n) \rightarrow E_{i}(\tilde{x}, n+1)$ is an isomorphism. For $v \in E_{i}(\tilde{x}, n) \backslash\{0\}$,

$$
\left\{\begin{array}{l}
\lim _{m \rightarrow \infty} \frac{1}{m} \log \left\|d_{x_{n}} f^{m}(v)\right\|=\lambda_{i}(\tilde{x}) \\
\lim _{m \rightarrow \infty}-\frac{1}{m} \log \left\|\left(\left.d_{x_{n-m}} f^{m}\right|_{E_{i}(\tilde{x}, n-m)}\right)^{-1}(v)\right\|=\lambda_{i}(\tilde{x})
\end{array}\right.
$$

3. if $i \neq j$ then

$$
\lim _{n \rightarrow \pm \infty} \frac{1}{n} \log \sin \angle\left(E_{i}(\tilde{x}, n), E_{j}(\tilde{x}, n)\right)=0,
$$

where $\angle(V, W)$ denotes the angle between subspaces $V$ and $W$.

4. $r(),. \lambda_{i}($.$) and m_{i}($.$) are measurable and \tilde{f}$-invariant. Moreover $r(\tilde{x})=r\left(x_{0}\right), \lambda_{i}(\tilde{x})=$ $\lambda_{i}\left(x_{0}\right)$ and $m_{i}(\tilde{x})=m_{i}\left(x_{0}\right)$ for all $i=1,2, \ldots, r(\tilde{x}) .(r(\tilde{x})$ is the number of subspaces on $T_{x_{n}} M$ for some $n \in \mathbb{Z}$ and for fixed $\tilde{x} \in M^{f}$.)

Observation 1.9. Any $\tilde{x} \in M^{f}$ with above property is called a Lyapunov Regular point (or just a Regular point) . By MET the set of all such regular points let us show it by $\tilde{\mathcal{R}}$, has a full $\tilde{\mu}$ measure and is $\tilde{f}$-invariant.

Definition 1.10 (Hyperbolic Measure). We call an ergodic measure $\mu$ a hyperbolic measure if:

- none of the Lyapunov exponents for $\mu$ are zero; 
- there exist Lyapunov exponents with different signs.

Observation 1.11. Notice that by number 4 of theorem 1.8, hyperbolicity of $\mu$ as a Borel probability measure on $M$ implies the hyperbolicity of $\tilde{\mu} \in \mathcal{M}_{\tilde{f}}\left(M^{f}\right)$. (Its corresponded measure on $M^{f}$ through proposition 1.1)

\subsection{Nonuniform-Hyperbolicity and Pesin Sets}

In the nonuniform version of hyperbolicity (Pesin Theory), instead of prescribing bounds for the expansion and contraction of vectors, we measure the asymptotic exponential behavior of points through long term derivatives.

Definition 1.12 (Non-Uniformly Hyperbolic Set). Let $f: M \rightarrow M$ be a $C^{2}$ endomorphism of a compact smooth Riemannian manifold $M$. An $\tilde{f}$-invariant Borel subset $\tilde{\Gamma} \subset M^{f}$ is said to be non-uniformly hyperbolic if exists

(a) numbers $\lambda$, $\mu$ such that $0<\lambda<1<\mu$;

(b) a number $\epsilon$ and Borel functions $C, K: \tilde{\Gamma} \rightarrow(0, \infty)$;

(c) subspaces $E^{s}(\tilde{x}, n)$ and $E^{u}(\tilde{x}, n)$ for each $\tilde{x}=\left(x_{n}\right) \in \tilde{\mathcal{R}}$, which satisfy the following conditions $\forall n \in \mathbb{Z}$ :

1. $T_{x_{n}} M=E^{s}(\tilde{x}, n) \oplus E^{u}(\tilde{x}, n)$,

2. $\left\{\begin{array}{l}d_{x_{n}} f E^{s}(\tilde{x}, n)=E^{s}(\tilde{f}(\tilde{x}), n)=E^{s}(\tilde{x}, n+1) ; \\ d_{x_{n}} f E^{u}(\tilde{x}, n)=E^{u}(\tilde{f}(\tilde{x}), n)=E^{u}(\tilde{x}, n+1)\end{array}\right.$

3. the subspace $E^{s}(\tilde{x}, n)$ is stable:

for $v \in E^{s}(\tilde{x}, n)$ and $m>0, \quad\left\|d_{x_{n}} f^{m}(v)\right\| \leq C(\tilde{x}) \lambda^{m} e^{\epsilon(m+|n|)}\|v\|$;

4. the subspace $E^{u}(x)$ is unstable:

for $v \in E^{u}(\tilde{x}, n)$ and $m<0, \quad\left\|d_{x_{n}} f^{m}(v)\right\| \leq C(\tilde{x}) \mu^{m} e^{\epsilon(|m|+|n|)}\|v\| ;$

5. $\angle\left(E^{s}(\tilde{x}, n), E^{u}(\tilde{x}, n)\right) \geq K(\tilde{x})$;

6. $C\left(\tilde{f}^{m}(\tilde{x})\right) \leq C(\tilde{x}) e^{\epsilon|m|}, \quad K\left(\tilde{f}^{m}(\tilde{x})\right) \geq K(\tilde{x}) e^{-\epsilon|m|}$.

In above definition if $E^{s}(\tilde{x}, n)$ is trivial, then we call $\tilde{\Gamma}$ a non-uniformly expanding set. By Multiplicative ergodic theorem or Oseledet's theorem we could guarantee that for every ergodic hyperbolic measure $\nu$ the set of Lyapunov regular points with nonzero exponents contains a non-uniformly hyperbolic set of full measure. From now on we 
suppose $\tilde{\mathcal{R}}$ to be this full measure set of regular points with non-zero Lyapunov exponents that is non-uniformly hyperbolic. Moreover for the sake of simplicity we always suppose our measures to be ergodic. let

$$
\mathcal{M}_{\text {erg }}^{*}(f):=\text { The space of all ergodic hyperbolic measures. }
$$

Definition 1.13 (Non-Uniformly Expanding Measure). We call an ergodic measure $\mu$ a non-uniformly expanding measure if both of the Lyapunov exponents are positive in $\mu-a . e$.

For a $\mu \in \mathcal{M}_{\text {erg }}^{*}(f)$ let introduce the Pesin Blocks. They let the behavior of Lyapunov exponents along the orbit of their points become under good controls. In other words "As we will see in next chapter under some metric change, they can be supposed as objects indicating uniformly hyperbolic behavior."

Assume

$$
E^{s}(\tilde{x}, n)=\bigoplus_{\lambda(\tilde{x})<0} E_{i}(\tilde{x}, n) \quad \& \quad E^{u}(\tilde{x}, n)=\bigoplus_{\lambda(\tilde{x})>0} E_{i}(\tilde{x}, n)
$$

Definition 1.14 (Pesin Blocks). Given $\lambda, \mu, \chi,>>\epsilon>0,1 \leq k \leq \operatorname{dim} M$ and for all $l>1$, we define a Pesin block $\tilde{\Delta}_{\chi, l}^{k}(\lambda, \mu, \epsilon)$ of $M^{f}$ consisting of $\tilde{x}=\left(x_{n}\right) \in M^{f}$ for which there exists a sequence of splittings $T_{x_{n}} M=E^{s}(\tilde{x}, n) \oplus E^{u}(\tilde{x}, n), n \in \mathbb{Z}$, satisfying:

- $\operatorname{dim} E^{u}(\tilde{x}, n)=k$;

- $d_{x_{n}} f\left(E^{s}(\tilde{x}, n)\right)=E^{s}(\tilde{x}, n+1), d_{x_{n}} f\left(E^{u}(\tilde{x}, n)\right)=E^{u}(\tilde{x}, n+1)$;

- for $m \geq 0$, for $v \in E^{s}(\tilde{x}, n)$ and $w \in E^{u}(\tilde{x}, n)$;

$$
\left\{\begin{array}{l}
\left\|d_{x_{n}} f^{m}(v)\right\| \leq e^{l \frac{\chi}{100}} e^{-\left(\lambda-\frac{\chi}{100}\right) m} e^{\left(\frac{\chi}{100}|n|\right)}\|v\|, \forall n \in \mathbb{Z}, n \geq 1 \\
\left\|\left(\left.d_{x_{n-m}} f^{m}\right|_{E^{u}(\tilde{x}, n-m)}\right)^{-1}(w)\right\| \leq e^{l \frac{\chi}{100}} e^{-\left(\mu-\frac{\chi}{100}\right) m} e^{\left(\frac{\chi}{100}|n-m|\right)}\|w\|, \forall n \in \mathbb{Z}, n \geq 1
\end{array}\right.
$$

and if $1 \leq k \leq \operatorname{dim} M-1$ then

- $\sin \angle\left(E^{s}(\tilde{x}, n), E^{u}(\tilde{x}, n)\right) \geq e^{-l \frac{\chi}{100}} e^{-\left(\frac{\chi}{100}\right)|n|}$.

Pesin blocks have some nice properties:

they are closed and therefore compact (due to compactness of $M^{f}$ ); $\tilde{\Delta}_{\chi, l}^{k} \subset \tilde{\Delta}_{\chi, l+1}^{k}$ and $\tilde{f}^{ \pm}\left(\tilde{\Delta}_{\chi, l}^{k}\right) \subset \tilde{\Delta}_{\chi, l+1}^{k}$;

the subspaces $E^{s}(\tilde{x}, n)$ and $E^{u}(\tilde{x}, n)$ of $T_{x_{n}} M$ depends on $\tilde{x}$ continuously over $\tilde{\Delta}_{\chi, l}^{k}$. 
Observation 1.15. In compare with definition 1.12 we see that in definition 1.14, we have $\lambda=e^{-\left(\lambda-\frac{\chi}{100}\right)}, \mu=e^{-\left(\mu-\frac{\chi}{100}\right)}$ and $\epsilon=\chi / 100$. Moreover, as we deal with hyperbolic ergodic measures with "SRB property"(see definition 1.34). By ergodicity assumption the Lyapunov exponents become a constant value for $\tilde{\mu}-$ a.e. point $\tilde{x} \in \tilde{\mathcal{R}}$. Therefore in the rest of the work we can suppose the $\chi$ in definition of a Pesin block to be

$$
\chi=\min _{i}\left|\lambda_{i}(x)\right|, \quad \text { for } 1 \leq i \leq 2(=\operatorname{dim} M)
$$

Since $\operatorname{dim} M=2$ by $S R B$ property assumption of the measure, the $k$ in definition 1.14 is always equal to one or two. Except chapter 3 in proof of theorem B that Pesin blocks become two dimensional and we will mention that $k$ is not one, we are assuming $k=1$ in the rest of the work. With no creating of ambiguity we may ignore $\chi$ and $k$ from the notation of Pesin blocks and show them by $\tilde{\Delta}_{l}$. Moreover we are always assuming the Pesin blocks having positive measure.

\subsection{Pesin Stable-Unstable Manifolds}

After the works of Pesin on developing a general theory on stable and unstable manifolds for diffeomorphisms [BP1],P.-D Liu and M. Qian developed a rigorous related theory for random diffeomorphisms and using similar techniques as given in [LQ], Sh. Zhu proves an unstable manifold theorem on non-invertible differentiable maps of finite dimension. Here we bring some important parts of Sh. Zhu work and we introduce the global stable and unstable sets useful in next chapters.[QXZ],[QZ]

Definition 1.16 (Local Unstable Manifold). For $\tilde{x} \in \tilde{\mathcal{R}}, \lambda_{i}(\tilde{x})>0$, we call $W_{\text {loc }}^{u}(\tilde{x}) a$ local unstable manifold of $f$ at $\tilde{x}$ when exists a $k$-dimensional $C^{2}$ embedded sub-manifold of $M$, such that there are numbers $\lambda>0,0<\epsilon<\lambda / 200,0<C$, and for any $y_{0} \in W_{\text {loc }}^{u}(\tilde{x})$, there exists a unique $\tilde{y}=\left\{y_{n}\right\}_{n \in \mathbb{Z}} \in M^{f}$ such that $\pi(\tilde{y})=y_{0}$ and $\forall n \in \mathbb{N}$,

$$
d\left(y_{-n}, x_{-n}\right) \leq C e^{-n(\lambda-\epsilon)} d\left(x_{0}, y_{0}\right)
$$

Moreover we define the local unstable set of $\tilde{f}$ at $\tilde{x}=\left(x_{n}\right)$ as

$$
\widetilde{W}_{l o c}^{u}(\tilde{x}):=\left\{\tilde{y} \in M^{f}: y_{0} \in W_{l o c}^{u}(\tilde{x}), d\left(y_{-n}, x_{-n}\right) \leq C e^{-n(\lambda-\epsilon)} d\left(x_{0}, y_{0}\right)\right\}
$$

Observation 1.17. It is remarkable that by above definition $\pi\left(\widetilde{W_{l o c}^{u}}(\tilde{x})\right)=W_{\text {loc }}^{u}(\tilde{x})$. 

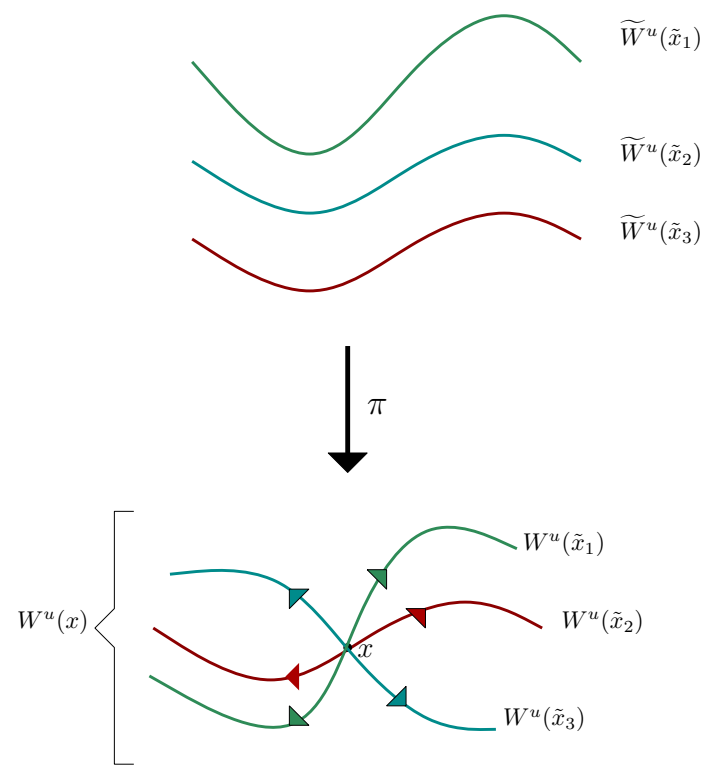

Figure 1.1: Unstable set and unstable manifolds of different trajectories .

Definition 1.18 (Unstable Manifold). The unstable manifold of $f$ corresponding to $\tilde{x} \in \tilde{\mathcal{R}}$ is defined as

$$
\left.W^{u}(\tilde{x})=\left\{y_{0} \in M \mid \exists \tilde{y} \in M^{f} \text { with } \pi \tilde{y}=y_{0}, \text { and } \varlimsup_{n \rightarrow+\infty} \frac{1}{n} \log d\left(x_{-n}, y_{-n}\right)<0\right\}\right\}
$$

and we will write

$$
\widetilde{W}^{u}(\tilde{x})=\left\{\tilde{y} \in M^{f} \mid \overline{\lim }_{n \rightarrow+\infty} \frac{1}{n} \log d\left(x_{-n}, y_{-n}\right)<0\right\}
$$

Notice that the unstable manifold corresponded to a trajectory $\tilde{x} \in \tilde{\mathcal{R}}$ can be defined as the union of an increasing sequence of images of $C^{1}$ embedded disks:

$$
W^{u}(\tilde{x})=\bigcup_{n=0}^{\infty} f^{n}\left(W_{l o c}^{u}\left(\tilde{f}^{-n}(\tilde{x})\right)\right) .
$$

Definition 1.19 (Global Unstable Set). We define the global unstable set of $f$ at a point $x$, as

$$
W^{u}(x):=\bigcup_{\tilde{x} \in \pi^{-1}(x) \cap \tilde{\mathcal{R}}} W^{u}(\tilde{x})
$$

For $x \in M$ and $r>0$, let define $T_{x} M(r)=\left\{\eta \in T_{x} M \mid\|\eta\|<r\right\}$. 
Proposition 1.20. For any Pesin block $\tilde{\Delta}_{l}$ on $M^{f}$, there is a continuous family of $C^{1}$ embedded $k$-dimensional discs $\left\{W_{\text {loc }}^{u}(\tilde{x})\right\}_{\tilde{x} \in \tilde{\Delta}_{l}}$ on $M$, together with positive numbers $\lambda_{l}, \epsilon_{l}<$ $\lambda_{l} / 200, r_{l}<1, \alpha_{l}, \beta_{l}$ such that the following properties hold for each $\tilde{x} \in \tilde{\Delta}_{l}$ :

- There is a $C^{1}$ map $h_{\tilde{x}}: O_{\tilde{x}} \rightarrow E_{\tilde{x}}^{s}$, where $O_{\tilde{x}}$ is an open subset of $E_{\tilde{x}}^{u}$ which contains $\left\{\eta \in E_{\tilde{x}}^{u}:|\eta|<\alpha_{l}\right\}$ satisfying

(a) $h_{\tilde{x}}(0)=0, D h_{\tilde{x}}(0)=0$;

(b) $\operatorname{Lip}\left(h_{\tilde{x}}\right) \leq \beta_{i} ; \operatorname{Lip}\left(D h_{\tilde{x}}\right) \leq \beta_{l}$, where $D h_{\tilde{x}}():. \eta \rightarrow D h_{\tilde{x}}(\eta)$;

(c) $\left\{W_{\text {loc }}^{u}(\tilde{x})\right\}=\exp _{x_{0}}\left(\operatorname{Graph}\left(h_{\tilde{x}}\right)\right)$.

- Let $W_{-n}(\tilde{x})=W_{l o c}^{u}\left(\tilde{f}^{-n} \tilde{x}\right)$ then $W_{-n+1} \subset f W_{-n}(\tilde{x})$ for all $n \geq 1$. Let $\rho_{0}>0$ be a positive real number such that the exponential map $\exp _{x}: T_{x} M\left(\rho_{0}\right) \rightarrow B\left(x_{0}, \rho_{0}\right):=$ $\{y \in M \mid d(y, x)<\rho\})$ is a $C^{\infty}$ diffeomorphism for every $x \in M$. Then exist numbers $r_{l} \in\left(0, \rho_{0} / 4\right), \epsilon_{l} \in(0,1)$ such that

- For any $r \in\left[r_{l} / 2, r_{l}\right]$ and each $\tilde{x} \in \tilde{\Delta}_{l}$, if $\tilde{z} \in \tilde{B}_{\tilde{\Delta}_{l}}\left(\tilde{x}, \epsilon_{l} r\right):=\left\{\tilde{y} \in \tilde{\Delta}_{l} \mid \tilde{d}(\tilde{x}, \tilde{y})<\epsilon_{l} r\right\}$, then $W_{\text {loc }}^{u}\left(\tilde{x}_{1}\right) \cap B\left(x_{0}, r\right)$ is connected and the map

$$
\tilde{z} \mapsto W_{l o c}^{u}(\tilde{z}) \cap B\left(x_{0}, r\right)
$$

is continuous from $\tilde{B}_{\tilde{\Delta}_{l}}\left(\tilde{x}, \epsilon_{l} r\right)$ to the space of subsets of $B\left(x_{0}, r\right)$ (endowed with the Hausdorff topology);

Observation 1.21. By above proposition we assume $\forall \tilde{x} \in \Delta_{l}$ the size of $W_{\text {loc }}^{u}(\tilde{x})$ is bounded away from zero and exists some $\alpha_{l}>0$ such that size $\left(W_{l o c}^{u}(\tilde{x})\right) \geq \alpha_{l}$.

Theorem 1.22. For any $\tilde{x} \in \tilde{\mathcal{R}}$ that $\lambda_{i}(\tilde{x})<0$ for some $1 \leq i \leq \operatorname{dim} M$, there exists a sequence of $C^{2}$ embedded $\operatorname{dim} E^{s}(\tilde{x})$-dimensional discs $\left\{W_{n}\right\}_{n=0}^{+\infty}$ satisfying some similar results as proposition 1.20 for stable manifolds(sets)such as:

- For each $n \in \mathbb{Z}^{+}$there exists a $C^{2}$ map

$$
h_{\tilde{f}^{n}(\tilde{x})}: O_{n}(\tilde{x}) \rightarrow E^{u}\left(\tilde{f}^{n}(\tilde{x})\right)
$$

where $O_{n}(\tilde{x})$ is an open subset of $E_{\tilde{f}^{n}(\tilde{x})}^{s}$, such that $h_{\tilde{x}}(0)=0, D h_{\tilde{x}}(0)=0$ and $W_{n}(\tilde{x})=\exp _{\tilde{f}^{n}(\tilde{x})}\left(\operatorname{graph}\left(h_{\tilde{x}}\right)\right)$;

- $f W_{n}(\tilde{x}) \subset W_{n+1}(\tilde{x})$;

- $\forall \tilde{x} \in \Delta_{l}$ the size of $W_{l o c}^{s}(\tilde{x})$ is bounded away from zero and exists $\alpha_{l}>0$ such that $\operatorname{size}\left(W_{l o c}^{s}(\tilde{x})\right) \geq \alpha_{l}$. 


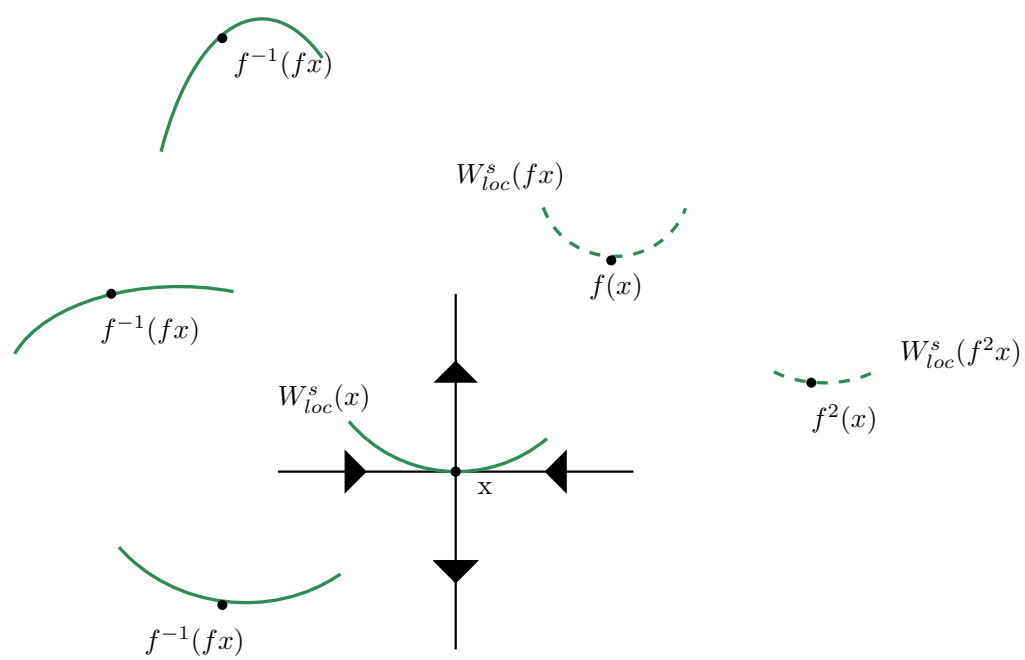

Figure 1.2: Stable sets and local stable manifolds.

and we may define

$W^{s}(\tilde{x})=\left\{y \in M \mid \varlimsup_{\lim _{n \rightarrow+\infty}} \frac{1}{n} \log d\left(f^{n} y, f^{n} x\right)<0\right\}$, if $\lambda_{i}(x)<0$ for some $1 \leq i \leq \operatorname{dim} M$ and otherwise $W^{s}(\tilde{x})=\left\{x_{0}\right\}$. In the other word for every $\tilde{x} \in \tilde{\mathcal{R}}$ with $\lambda_{i}(\tilde{x})<0$

$$
W^{s}(\tilde{x})=\bigcup_{n=0}^{+\infty} f^{-n}\left(W_{n}(\tilde{x})\right)
$$

Observation 1.23. We call $W^{s}(\tilde{x})$ the Stable Set of $f$ corresponded to $\tilde{x} \in \pi^{-1}(x)$ (for some base point $x \in M)$. Notice that $\forall \tilde{x} \in \pi^{-1}(x)$ all trajectories have the same forward orbit. This means that in fact stable sets are independent of the choice of pre-images and for any $\tilde{y} \in \pi^{-1}(x)$ we have

$$
W^{s}(\tilde{y})=W^{s}(x)
$$

Therefore we may use the notation $W^{s}(x)$ instead of $W^{s}(\tilde{x})$. As it is observable in fig 1.2, stable sets are expressed as a countable union of $C^{1}$ curves on $M$.

\subsection{Absolute Continuity and Conditional Measures}

An important notion behind the criteria used in [HHTU] and consequently this work, is absolute continuity. Let $\zeta$ be a partition of the manifold $M$. We call $\zeta$ a measurable partition if the quotient space $M / \zeta$ is separated by a countable number of measurable sets. This section also plus some changes is from chapter $\mathrm{V}$ of [QXZ]. 
We have the following definition for absolute continuity of measures:

Definition 1.24 (Absolute Continuity). Given a measurable space $(X, \mathcal{B})$, we say that for $\mu$ and $\nu$ two measures on $(X, \mathcal{B}), \nu$ is absolutely continuous with respect to $\mu$ if $\nu(A)=0$ for any $A \in \mathcal{B}$ such that $\mu(A)=0$. The absolute continuity of $\nu$ with respect to $\mu$ is usually denoted by $\nu \ll \mu$.

Definition 1.25. Let $X$ be a compact metric space and $\mathcal{P}$ be any measurable partition of $X$. Suppose $\pi: X \rightarrow \mathcal{P}$ is the map associating to each $x \in X$ the atom $P \in \mathcal{P}$ that contains $x$ and let $\hat{\mu}=\pi_{*} \mu$. Then a system of conditional measures of $\mu$ with respect to $\mathcal{P}$ is a family $\left(\mu_{P}\right)_{P \in \mathcal{P}}$ of probability measures on $X$ such that

- $\mu_{P}(P)=1$ for $\hat{\mu}$-almost every $P \in \mathcal{P}$.

- For any given continuous $\phi: X \rightarrow \mathbb{R}$, the function $P \rightarrow \int \phi d \mu_{P}$ is measurable and $\int \phi d \mu=\int\left(\int \phi d \mu_{P}\right) d \hat{\mu}(P)$.

Theorem 1.26 (Rokhlin [R1]). If $\mathcal{P}$ is a measurable partition, then there exists some system of conditional measures of $\mu$ relative to $\mathcal{P}$.

A corollary of the definition of conditional measure is the following basic proposition from [QXZ],[LQ].

Proposition 1.27. Let $(X, \mathcal{B}, m)$ be a Lebesgue space and let $\mathcal{P}$ be a measurable partition of $X$. If $\nu$ is another probability measure on $\mathcal{B}$ which is absolutely continuous with respect to $m$, then for $\nu$-almost all $x \in X$ the conditional measure $\nu_{P(x)}$ is absolutely continuous with respect to $m_{P(x)}$.

Now Let $\tilde{\Delta}_{l}$, be an arbitrarily chosen Pesin block and $\tilde{x} \in \tilde{\Delta}_{l}$. For $x \in \Delta_{l}=\pi\left(\tilde{\Delta}_{l}\right)$ and for sufficiently small $r>0$ put

$$
U_{\Delta_{l}}(\tilde{x}, r)=\exp _{\tilde{x}}\left\{\eta \in T_{x} M:\|\eta\|_{\tilde{x}}<r\right\}
$$

(Definition of $\|\cdot\|_{\tilde{x}}$ is given in next chapter and $\exp : T_{x} M \rightarrow B(x, r) \subset M$. )

and $\mathcal{F}_{\Delta_{l}}(\tilde{x}, r)$ the collection of all local stable-manifolds $W_{l o c}^{s}(y)$ passing through $y \in$ $W_{l o c}^{u}(\tilde{x})$ and $\tilde{x} \in \tilde{\Delta}_{l}$.

Definition 1.28. Sub-manifold $W$ of $M$ is called transversal to $\mathcal{F}_{\Delta_{l}}(\tilde{x}, r)$ if the following holds true: 
- $W \subset U_{\Delta_{l}}(\tilde{x}, r)\left(r\right.$ can be found through 1.20) and $\exp _{\tilde{x}}^{-1} W$ is the graph of a $C^{1}$ map.

- W intersects $W^{s}(y) \in \mathcal{F}_{\Delta_{l}}(\tilde{x}, r)$ at exactly one point and this intersection is transversal, i.e. $T_{z} W \oplus T_{z} W^{s}(y)=T_{z} M$ where $z=W \cap W^{s}(y)$.

Moreover for a periodic point $p$ when $W$ is a part of $\bar{p}$-unstable manifold intersecting the stable set transversally, we say that exists a transversal homoclinic point. (Recall that $\bar{p}=\left(\ldots p f^{m-1}(p) \ldots f(p) p, \ldots\right)$ for $m$ being the period of $\left.p.\right)$

Consider now two sub-manifolds $W^{1}$ and $W^{2}$ transversal to $(\tilde{x}, r)$. By theorem 1.22, $\left\{W_{l o c}^{s}(y)\right\}_{y \in W_{l o c}^{u}(\tilde{x})}$ is a continuous family of $C^{1}$ embedded disks with $\operatorname{size}\left(W_{l o c}^{s}(y)\right)>\alpha_{1}$. There exist two open sub-manifolds $\widehat{W}^{1}$ and $\widehat{W}^{2}$ such that we can well define a so-called Holonomy map that

$$
\begin{aligned}
& \mathcal{H}: \widehat{W}^{1} \cap \mathcal{F}_{\Delta_{l}}(\tilde{x}, r) \longrightarrow \widehat{W}^{2} \cap \mathcal{F}_{\Delta_{l}}(\tilde{x}, r) \\
& z \longmapsto \\
& \widehat{W}^{2} \cap W_{l o c}^{u}(y) .
\end{aligned}
$$

Whenever $z=\widehat{W}^{1} \cap W_{l o c}^{s}(y)$ and $y \in \Delta_{l}$.

Definition 1.29. The family $\mathcal{F}_{\Delta_{l}}(\tilde{x}, r)$ is said to be absolutely continuous if every holonomy map constructed as above is absolutely continuous with respect to $m_{W^{1}}$ and $m_{W^{2}}$.(i.e. holonomy maps are measurable and take Lebesgue zero sets of $W^{1}$ into Lebesgue zero sets of $W^{2}$.)

There are results from [LQ](theorem iii,5,1) that ensures the absolute continuity of the family $\mathcal{F}_{\Delta_{l}}(\tilde{x}, r)$.

\subsubsection{Lambda Lemma}

We use Lambda lemma in different places during the proof of main theorem.

Definition 1.30 (Homoclinic Equivalent Classes). Two hyperbolic periodic points $p$ and $q$ are in a transversal homoclinical relation if $W^{s}(O(p)) \pitchfork W^{u}(\bar{q}) \neq \emptyset \neq W^{s}(O(q)) \pitchfork W^{u}(\bar{p})$ and it is denoted by $p \sim q$. The class of all such periodic points in a transversal homoclinical relation with some hyperbolic periodic point p, we call Homoclinic Equivalent Class of $p$ and denote it by $[p]$.

Lemma 1.31 ( $\lambda$-Lemma[R2]). If $p$ is a hyperbolic fixed point of map $f$, and $\Sigma$ is an embedded $C^{1}$ sub-manifold of $M$ intersecting $W_{\delta}^{s}(p)$ transversally, near $p$ then for $n$ large enough $f^{n}(\Sigma)$ contains an embedded manifold $\Sigma_{n}$ which is $C^{1}-$ close to $W_{\delta}^{u}(\bar{p})$ where $\bar{p}=(\ldots p p p p \ldots) \in M^{f}$. 


\subsection{SRB Property and Main Results}

Let $f: M \rightarrow M$ a $C^{2}$ endomorphism and $M$ closed Riemannian manifold and $\mu \in$ $\mathcal{M}_{f}(M)$. The $\tilde{f}$-invariant Borel probability measure on $M^{f}$ corresponding to $\mu$ we denote it with $\tilde{\mu}$.

Definition 1.32 (Unstable Partition Sub-Ordinate to $W^{u}$ ). A measurable partition $\zeta$ of $M^{f}$ is said to be subordinate to $W^{u}$ manifolds of $(f, \mu)$ if for $\tilde{\mu}$-a.e. $\tilde{x}, \zeta(\tilde{x})$ has the following properties:

- $\pi_{\mid} \zeta(\tilde{x}): \zeta(\tilde{x}) \rightarrow \pi(\zeta(\tilde{x}))$ is bijective

- There exists a $k(\tilde{x})$ dimensional $C^{2}$ embedded sub-manifold $W_{\tilde{x}}$ of $M$, such that $W_{\tilde{x}} \subset W^{u}(\tilde{x}), \pi(\zeta(\tilde{x})) \subset W_{\tilde{x}}$ and $\pi(\zeta(\tilde{x}))$ contains an open neighborhood of $x_{0}$ in $W_{\tilde{x}}$, this neighborhood being taken in the topology of $W_{\tilde{x}}$ as a sub-manifold of $M$.

Using the local unstable sets $\widetilde{W}_{\text {loc }}^{u}(\tilde{x})$ of $f$ in $M^{f}$, it is possible to construct a suitable measurable partition of $M^{f}$ subordinate to $W^{u}$-manifolds. For this purpose the following $\sigma$-algebra will be considered:

$$
\mathcal{B}^{u}=\left\{B \in \mathcal{B}_{\tilde{\mu}}\left(M^{f}\right) \mid \tilde{x} \in B \text { implies } \widetilde{W}^{u}(\tilde{x}) \subset B .\right\}
$$

Where $\mathcal{B}_{\tilde{\mu}}\left(M^{f}\right)$ is the completion of $\mathcal{B}\left(M^{f}\right)$ with respect to $\tilde{\mu}$. It fact it consists the measurable subsets of $M^{f}$ which are unions of some global unstable sets.

Following proposition of [QZ] shows that there exists a measurable partition of $M^{f}$ sub-ordinate to $W^{u}$-manifolds of $f$.

Proposition 1.33. There exists a measurable partition $\eta$ of $M^{f}$ which has the following properties:

- $\tilde{f}^{-1} \eta \geq \eta$

- $\bigvee_{n=0}^{+\infty} \tilde{f}^{-n} \eta$ is equal to the partition into single points.

- $\mathcal{B}\left(\bigwedge_{n=0}^{+\infty} \tilde{f}^{n} \eta\right)=\mathcal{B}^{u}, \tilde{\mu}-\bmod 0$, where $B(\eta)$ is the $\sigma$-algebra consisting of all measurable $\eta$-sets for a measurable partition $\eta$ of $M^{f}$.

Definition 1.34 (SRB Property). We say that the $f$-invariant measure $\mu$ has the $S R B$ property, if for every measurable partition $\zeta$ of $M^{f}$ subordinate to $W^{u}$-manifolds of $(f, \mu)$ we have, for $\tilde{\mu}$-a.e. $\tilde{x} \in M^{f}$,

$$
\pi\left(\tilde{\mu}_{\tilde{x}}^{\zeta}\right) \prec \prec m_{\tilde{x}}^{u}
$$




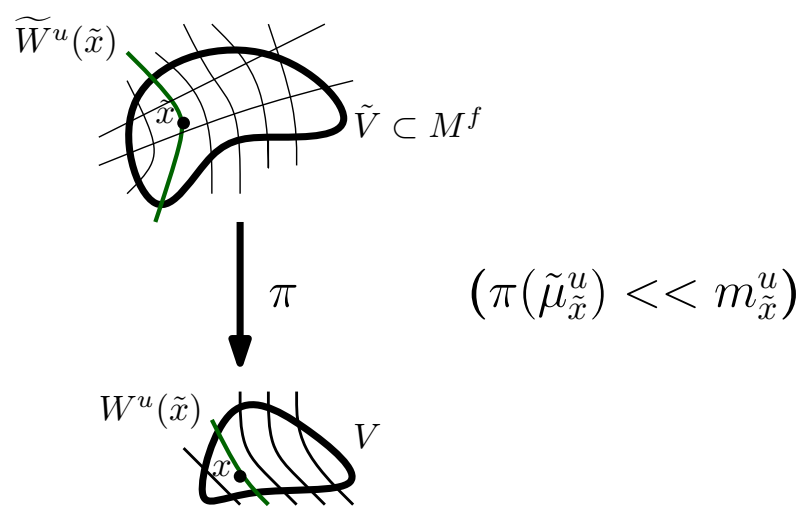

Figure 1.3: SRB Property

where $\left\{\tilde{\mu}_{\tilde{x}}^{\zeta}\right\}_{\tilde{x} \in M^{f}}$ is a canonical system of conditional measures of $\tilde{\mu}$ associated with $\zeta$, $\pi\left(\tilde{\mu}_{\tilde{x}}^{\zeta}\right)$ is the projection of $\tilde{\mu}_{\tilde{x}}^{\zeta}$ under $\pi_{\mid} \zeta(\tilde{x}): \zeta(\tilde{x}) \rightarrow \pi(\zeta(\tilde{x}))$ and $m_{\tilde{x}}^{u}$ denotes the Lebesgue measure on $W_{l o c}^{u}(\tilde{x})$ induced by its inherited Riemannian structure.

In In figure 1.3 , let $\tilde{V} \subset M^{f}$ a Borel subset with $\tilde{\mu}(\tilde{V})>0$. Suppose that $\tilde{V}$ is the disjoint union of a continuous family $\widetilde{W}^{u}$ - discs in the form of $\widetilde{W}_{l o c}^{u}(\tilde{x})$ from proposition 1.33. Then $\tilde{\mu}_{\tilde{x}}^{u}$ is the conditional probability measure of $\tilde{\mu}$ on the element of partition containing $\tilde{x}$.

We also have the following Pesin entropy formula for endomorphisms from [QZ].

Theorem 1.35. Let $f$ be a $C^{2}$ endomorphism on $M$ with an $f$-invariant Borel probability measure $\mu$ satisfying the integrability condition (??). Then the entropy formula holds

$$
h_{\mu}(f)=\int_{M} \Sigma_{i} \lambda_{i}(x)^{+} m_{i}(x) d \mu(x)
$$

if and only if $\mu$ has the SRB property.

There is an Important Remark in this work that we use it in different part of the proves of theorems. We bold it here.

Place $\eta$ as a measurable partition of $M^{f}$ sub-ordinate to $W^{u}$ of $f$ with respect to $\mu$ a hyperbolic ergodic measure with SRB property. Let $\left\{\tilde{\mu}_{\tilde{x}}^{u}\right\}$ be the canonical system of conditional measures of $\tilde{\mu}$ associated with partition $\eta$ and $m_{\tilde{x}}^{u}$ be the Lebesgue measure on $W_{\tilde{x}}$ induced by its Riemannian structure as a sub-manifold of $M$. Let

$$
J^{u}(\tilde{x}):=\left|\operatorname{det}\left(\left.d_{x} f\right|_{E_{\tilde{x}}^{u}}\right)\right|
$$

for $\tilde{\mu}$-a.e $\tilde{x} \in M^{f}$. Then we can define a density function $\rho_{\tilde{x}}$ for $\pi \tilde{\mu}_{\tilde{x}}^{u}$ with respect to $m_{\tilde{x}}^{u}$ 
such that

$$
\Theta(\tilde{x}, \tilde{y}):=\frac{\rho(\tilde{x})}{\rho(\tilde{y})}=\prod_{k=1}^{+\infty} \frac{J^{u}\left(\tilde{f}^{-k}(\tilde{x})\right)}{J^{u}\left(\tilde{f}^{-k}(\tilde{y})\right)} .
$$

By lemma VII.9.1 of [QXZ]:

Lemma 1.36. For $\tilde{\mu}$-a.e. $\tilde{x}, \tilde{y}$ the $\Theta(\tilde{x}, \tilde{y})$ is well-defined Lipschitz function on $\eta(\tilde{x})$ and is uniformly bounded away from 0 and $\infty$ on $\eta(\tilde{x})$.

Observation 1.37 (Important Remark). F. Ledrappier and L.-S. Young [LY] showed first for diffeomorphisms that for measures which accept Pesin entropy formula, the densities $d \mu_{x}^{u} / d m_{x}^{u}$ are given by strictly positive functions that are $C^{1}$ along unstable manifold. ( $\mu_{x}^{u}$ is the unstable conditional measure and $m_{x}^{u}$ the induced Lebesgue inherited from Riemannian structure.) Using above lemma on endomorphisms, from now on we assume the unstable conditional measures on $M$, to be similar with Lebesgue $W^{u}$-conditionals induced from Riemannian structure.

\subsubsection{Ergodic Component and SRB Property}

However the base of this work is settled on the assumption of ergodic hyperbolic measures, but also in the lack of ergodicity for measures, using ergodic decomposition theorem $[\mathrm{KH}]$, theorem 3.17 and proposition 1.39 we can obtain the same result. We use Margulis-Ruelle inequality which is an important concept that connects metric entropy with Lyapunov exponents. Following theorem II.1.1 of [QXZ] gives a version of MargulisRuelle inequality for $C^{1}$ maps.

Theorem 1.38. Lef $f$ be a $C^{1}$ map of a compact, smooth Riemannian manifold $M$. If $\mu$ is an $f$-invariant Borel probability measure on $M$, then

$$
h_{\mu}(f) \leq \int_{M} \sum_{i} \lambda_{i}^{+}(x) m_{i}(x) d \mu(x),
$$

where $-\infty<\lambda_{1}(x)<\cdots<\lambda_{r(x)}<\infty$ are Lyapunov exponents of $f$ at $x$ and $m_{i}(x)$ is the multiplicity of $\lambda_{i}(x)$ for each $i=1,2, . ., r(x)$.

Proposition 1.39. Almost all ergodic components of $\mu$ are hyperbolic and SRB.

Proof. The hyperbolicity is easy to see because if not it is possible to find a positive measure set, where $\mu$ is not hyperbolic and this is a contradiction. For SRB property case, we know that by ergodic decomposition and Margulis-Ruelle inequality, there exists 
a probability measure $\hat{\mu}$ in the space of all probability measures with supp in ergodic measures $\mathcal{M}(f)$, such that $h_{\mu}=\int_{\mathcal{M}(f)} h_{\nu} d \hat{\mu}(\nu) \leq \int \sum_{i} \lambda_{i}^{+}(x) d \mu$ counting multiplicities. From the other side by theorem VII.1.1 of book [QXZ] $\mu$ has SRB property if and only if

$$
h_{\mu}(f)=\int_{M} \sum_{i} \lambda_{i}^{+}(x) d \mu(x)
$$

They clearly imply that $\hat{\mu}$-almost every $\nu$ will satisfy the entropy formula and so it has SRB property. 


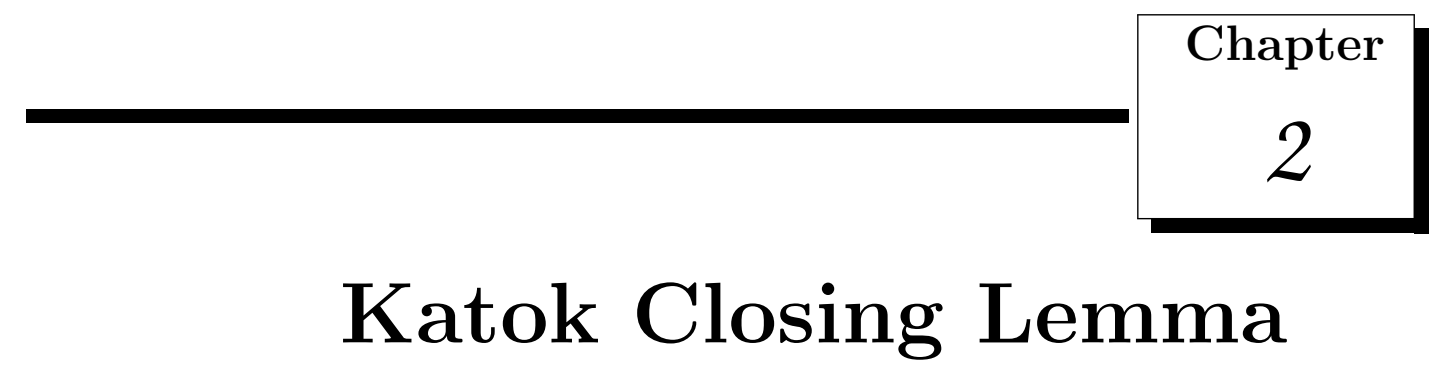

In this chapter we are going to give the definition of Lyapunov metrics near regular points which give us the facility of considering the linear parts of $f$ along the trajectory of such a point as a uniformly hyperbolic operator. After that we will see the Katok version of closing lemma for non-singular endomorphisms (local diffeomorphisms), and consequently the existence of transversal homoclinic intersection for a hyperbolic periodic point derived from closing lemma. This construction is mostly based on Katok's paper [K1]. The proof is similar to diffeomorphism case.

\subsection{Lyapunov charts}

Let $f$ be a $C^{2}$ - endomorphism of a closed Riemannian surface $M$. Assume that we have a non-empty Pesin Block $\tilde{\Delta}_{l}$ for $l>1$. We can change the metric on $\tilde{\Delta}_{l}$ so that $\left.f\right|_{\tilde{\Delta}_{l}}$ looks uniformly hyperbolic. This happens by replacing the induced Riemannian metric on $T_{x_{n}} M\left(\tilde{x}=\left(x_{n}\right) \in \tilde{\Delta}_{l}\right)$ by a new metric. It removes the need for constants $e^{l \chi / 100}$ in measuring the hyperbolicity of each $\tilde{\Delta}_{l}$. Let choose real numbers $0<\hat{\lambda}<\dot{\mu}<\infty$ such that

$$
\grave{\lambda}=\lambda-2 \epsilon, \quad \dot{\mu}=\mu-2 \epsilon .
$$

We can define the new metric $<., .>_{\tilde{x}}^{\prime}$ as:

$$
<v_{s}, w_{s}>_{\tilde{x}}^{\prime}:=\sum_{m=0}^{\infty}<d f^{m}\left(v_{s}\right), d f^{m}\left(w_{s}\right)>_{x_{n}} e^{2 \lambda^{\prime} m}
$$


where $v_{s}, w_{s} \in E^{s}(\tilde{x}, 0)$, and

$$
<v_{u}, w_{u}>_{\tilde{x}}^{\prime}:=\sum_{m=0}^{\infty}<d f^{-m}\left(v_{u}\right), d f^{-m}\left(w_{u}\right)>_{x_{-n}} e^{2 \lambda^{\prime} m}
$$

where $v_{u}, w_{u} \in E^{u}(\tilde{x}, 0)$. Now for $(v, w) \in T_{x_{0}} M$ that $v=v_{s}+v_{u}, w=w_{s}+w_{u}$, let define

$$
<v, w>_{\tilde{x}}^{\prime}:=\max \left\{<v_{s}, w_{s}>_{\tilde{x}}^{\prime},<v_{u}, w_{u}>_{\tilde{x}}^{\prime}\right\}
$$

The new metric induces a new norm $\|\cdot\|_{\tilde{x}}^{\prime}$ on $T_{x_{0}} M$ :

$$
\begin{aligned}
& \left\|v_{s}\right\|_{\tilde{x}}^{\prime}=\left(\sum_{m=0}^{\infty} e^{2 \lambda^{\prime} m}\left\|d_{x_{0}} f^{m}\left(v_{s}\right)\right\|^{2}\right)^{1 / 2}, \\
& \left\|v_{u}\right\|_{\tilde{x}}^{\prime}=\left(\sum_{m=0}^{\infty} e^{\mu^{\prime} m}\left\|\left(\left.d_{x_{0}} f^{m}\right|_{E^{u}(\tilde{x}, 0)}\right)^{-1}\left(v_{u}\right)\right\|^{2}\right)^{1 / 2}, \\
& \|v\|_{\tilde{x}}^{\prime}=\max \left\{\left\|v_{s}\right\|_{\tilde{x}}^{\prime},\left\|v_{u}\right\|_{\tilde{x}}^{\prime}\right\} .
\end{aligned}
$$

Observe that above series converges. For instance if $\tilde{x} \in \tilde{\Delta}_{l}$ then by 2.1 and definition 1.14 taking $\epsilon=\frac{\chi}{100}$,

$$
\begin{aligned}
\left\|v_{s}\right\|_{\tilde{x}}^{\prime 2} & =\sum_{m=0}^{\infty} e^{2(\lambda-2 \epsilon) m}\left\|d_{x_{0}} f^{m}\left(v_{s}\right)\right\|^{2} \\
& \leq \sum_{m=0}^{\infty} e^{2(\lambda-2 \epsilon) m} e^{2 \epsilon l} e^{-2(\lambda-\epsilon) m}\left\|v_{s}\right\|^{2} \\
& =\left\|v_{s}\right\|^{2} e^{2 \epsilon l}\left(\sum_{m=0}^{\infty} e^{-\epsilon m}\right)^{2} \\
& \Rightarrow\left\|v_{s}\right\|_{\tilde{x}}^{\prime} \leq\left\|v_{s}\right\| e^{\frac{l \chi}{100}} \sum_{m=0}^{\infty} e^{\frac{-\chi}{100} m}<\infty
\end{aligned}
$$

Similarly for $\left\|v_{u}\right\|_{\tilde{x}}^{\prime}$. As it was mentioned in above lines, the new metric exhibits a local "hyperbolicity" which is independent of $\tilde{x} \in \tilde{\Delta}_{l}$. For example we see that for $v_{s} \in E^{s}(\tilde{x}, 0), v_{u} \in E^{u}(\tilde{x}, 0):$

$$
\left\|d_{x_{0}} f\left(v_{s}\right)\right\|_{\tilde{f}(\tilde{x})}^{\prime} \leq e^{-\frac{98}{100} \chi}\left\|v_{s}\right\|_{\tilde{x}}^{\prime}
$$




$$
\left\|d_{x_{0}} f\left(v_{u}\right)\right\|_{\tilde{f}(\tilde{x})}^{\prime} \geq e^{\frac{98}{100} \chi}\left\|v_{u}\right\|_{\tilde{x}}^{\prime}
$$

Also exist the following estimate on the norms (\|.\| is the induced Riemannian norm on $\left.T_{x} M\right)$.

$$
\frac{1}{2}\|v\| \leq\|v\|_{\tilde{x}}^{\prime} \leq a_{l}\|v\| \quad \forall \tilde{x}=\left(x_{n}\right) \in \tilde{\Delta}_{l}
$$

where $a_{l}=C e^{\frac{l \chi}{100}}$ which $C=\sum_{n=0}^{\infty} e^{-\frac{\chi}{100} n}$. The right hand estimate is observable through 2.5 and for the left hand estimate we observe that if $v=\left(v_{s}, v_{u}\right) \in T_{x} M$ then $\|v\|^{2} \leq$ $\left(\left\|v_{s}\right\|+\left\|v_{u}\right\|\right)^{2}$. Using the definition of Lyapunov norm we have

$$
\left\|v_{s}\right\| \leq\left\|v_{s}\right\|_{\tilde{x}}^{\prime} \text { and } \quad\left\|v_{u}\right\| \leq\left\|v_{u}\right\|_{\tilde{x}}^{\prime}
$$

Without any loss of generality suppose that in $2.4,\|v\|_{\tilde{x}}^{\prime}=\left\|v_{s}\right\|_{\tilde{x}}^{\prime}$. Then

$$
\|v\|^{2} \leq\left\|v_{s}\right\|_{\tilde{x}}^{2}+\left\|v_{s}\right\|_{\tilde{x}}^{2}+2\left\|v_{s}\right\|_{\tilde{x}}^{\prime}\left\|v_{s}\right\|_{\tilde{x}}^{\prime}=4\|v\|_{\tilde{x}}^{2} \Rightarrow \frac{1}{2}\|v\| \leq\|v\|_{\tilde{x}}^{\prime}
$$

The $\|.\|_{\tilde{x}}^{\prime}$ is called Lyapunov Norm. The following proposition 2.3 of [K1] is about the existence of Lyapunov charts.(Figure 2.1)

Proposition 2.1. There exists a number $r>0$ so that for every point $\tilde{x} \in\left(\tilde{\mathcal{R}}=\bigcup_{l} \Delta_{l}\right)$ we can find a neighborhood $B(\tilde{x})$ around the point $x=\pi(\tilde{x})$ and a diffeomorphism $\Phi_{\tilde{x}}$ : $B_{r} \times B_{r} \rightarrow B(\tilde{x})\left(B_{r}\right.$ is Euclidean closed $r$-disc around the origin in $\left.\mathbb{R}\right)$. Also there exists a family of $C^{1}$ maps $F_{\tilde{x}}: B_{r} \times B r \rightarrow \mathbb{R} \times \mathbb{R}$ satisfying the following properties:

1. $\Phi_{\tilde{x}}(0)=\pi(\tilde{x})$

2. $F_{\tilde{x}}(z)=\Phi_{\tilde{f}(\tilde{x})}^{-1} \circ f \circ \Phi_{\tilde{x}}(z) \quad\left(\right.$ for $\left.z=(u, v) \in B_{r} \times B_{r}\right)$;

3. $F_{\tilde{x}}$ has the form:

$$
F_{\tilde{x}}(u, v)=\left(A_{\tilde{x}} u+h_{\tilde{x}}^{1}(u, v), B_{\tilde{x}} v+h_{\tilde{x}}^{2}(u, v),\right.
$$

such that:

$$
h_{\tilde{x}}^{2}(0,0)=h_{\tilde{x}}^{2}(0,0)=0, d h_{\tilde{x}}^{1}(0,0)=d h_{\tilde{x}}^{2}(0,0)=0
$$

and

$$
\left\|A_{\tilde{x}}\right\| \leq e^{\frac{-99}{100} \chi}, \quad\left\|B_{\tilde{x}}\right\| \geq e^{\frac{99}{100} \chi} .
$$

(all the norms are considered as Euclidean.) 


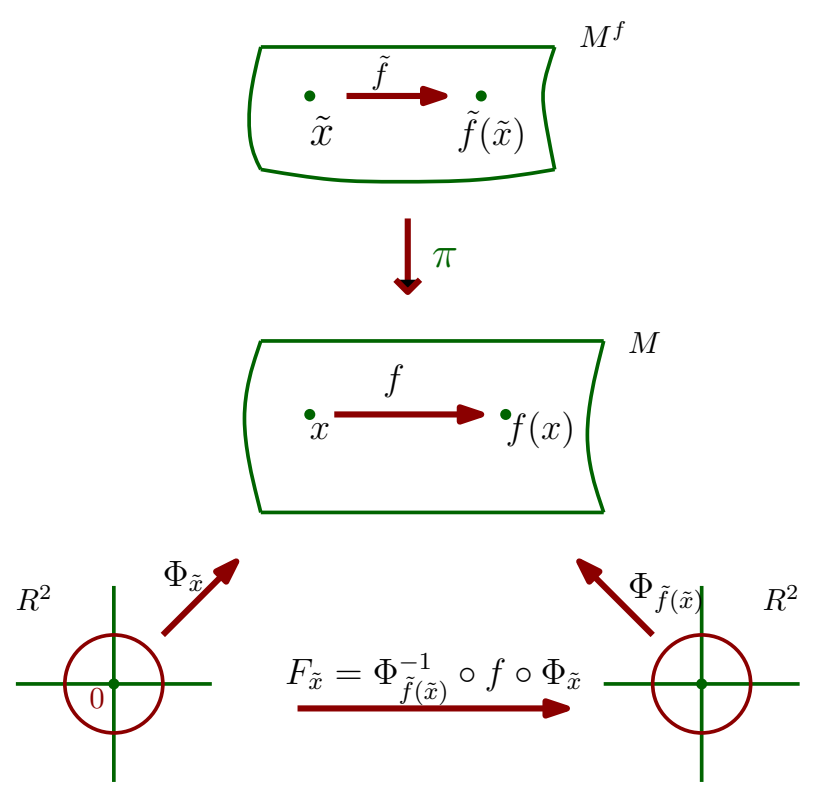

Figure 2.1: Lyapunov Charts

For $z \in B_{r} \times B_{r}$ let $h_{\tilde{x}}(z)=\left(h_{\tilde{x}}^{1}(z), h_{\tilde{x}}^{2}(z)\right)$, then $\left\|\left(d h_{\tilde{x}}\right)_{z_{1}}-\left(d h_{\tilde{x}}\right)_{z_{2}}\right\| \leq \Upsilon a_{l}\left\|z_{1}-z_{2}\right\|$ where $\Upsilon$ is an absolute constant.

4. the metric $\|\cdot\|_{\tilde{x}}^{\prime}$ depends continuously on $\tilde{x}$ over any set $\tilde{\Delta}_{l}$.

5. for any $z \in M$ the decomposition $T_{z} M=d \Phi_{\tilde{x}} \mathbb{R} \times d \Phi_{\tilde{x}} \mathbb{R}$ depends continuously on $\tilde{x}$ for such $\tilde{x} \in \tilde{\Delta}_{l}$ that $z \in B(\tilde{x})$.

We may diminish the size of the neighborhood $B(\tilde{x})$ and change it to $R(\tilde{x})=\Phi_{\tilde{x}}\left(B_{\eta_{l}} \times\right.$ $\left.B_{\eta_{l}}\right)$. Let suppose

$$
\lambda(\chi)=\max \left\{1 / 2, e^{\frac{-99}{100} \chi},\right\}
$$

then for $\tilde{x} \in \tilde{\Delta}_{l}$,

$$
\eta_{l}=\frac{(1-\lambda(\chi))^{2}}{100}(2 \Upsilon)^{-1}\left(a_{l}^{-1}\right)
$$

For $z=(u, v) \in \Phi_{\tilde{x}}^{-1}(R(\tilde{x}))$ using above estimation we gain the following estimate for the non-linear part of $F_{\tilde{x}}$.

$$
\left\|\left(d h_{\tilde{x}}\right)_{z}\right\| \leq \Upsilon a_{l}\|z\| \leq \frac{(1-\lambda(\chi))^{2}}{100}
$$

(Notice that $\eta_{l}$ is not depending on $\tilde{x} \in \tilde{\Delta}_{1}$.)

This new $R(\tilde{x})$ is called standard $\tilde{x}$-box or Lyapunov chart. 


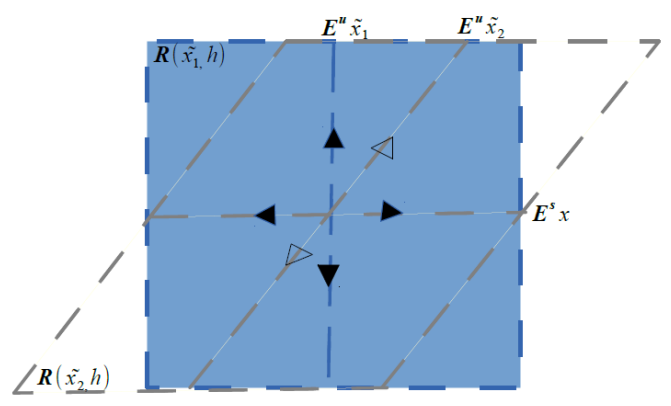

Figure 2.2: unstable manifolds for $\tilde{x}_{1} \neq \tilde{x}_{2}$ such that $\pi\left(\tilde{x}_{1}\right)=\pi\left(\tilde{x}_{2}\right)=x$.

\subsection{Admissible Manifolds}

Fix some $0<h \leq 1$ and for some $\tilde{x} \in \tilde{\Delta}_{l}$ when necessary we can $\operatorname{shrink} R(\tilde{x})$ uniformly to $R(\tilde{x}, h)=\Phi_{\tilde{x}}\left(B_{h \eta_{l}} \times B_{h \eta_{l}}\right)$. Then for $0<\gamma<1, \delta \geq 0,0<h \leq 1$, the set of one-dimensional admissible $(s, h, \tilde{x})$-manifolds and one dimensional admissible $(u, h, \tilde{x})-$ manifolds near the point $x$ on $R(\tilde{x}, h)$, will be defined respectively as:

$$
\begin{gathered}
S_{\tilde{x}}^{\gamma, \delta, h}=\left\{\Phi_{\tilde{x}}(\operatorname{graph} \phi) \mid \phi \in C^{1}\left(B_{h \eta_{l}}^{s}, B_{h \eta_{l}}^{u}\right),\|\phi(0)\| \leq \delta,\|d \phi\| \leq \gamma\right\} \\
U_{\tilde{x}}^{\gamma, \delta, h}=\left\{\Phi_{\tilde{x}}(\operatorname{graph} \phi) \mid \phi \in C^{1}\left(B_{h \eta_{l}}^{u}, B_{h \eta_{l}}^{s}\right),\|\phi(0)\| \leq \delta,\|d \phi\| \leq \gamma\right\} .
\end{gathered}
$$

Let $d(.,$.$) be the distance function generated by the given Riemannian metric on M, \tilde{d}(.,$. the corresponding distance function on $M^{f}$ as defined in 1.1 and $d_{\tilde{x}}^{\prime}(.,$.$) the Lyapunov$ distance generated by metric $<., .>_{\tilde{x}}^{\prime}$.

Following proposition shows that for a $\gamma$ and any sufficiently small $\delta$ then $U_{\tilde{x}}^{\gamma, \delta, h}$ is $f$-invariant and every admissible manifold belonging to that, is expanding. For $\chi>0$ and $\lambda=\lambda(\chi)$, let $\gamma=\gamma(\chi)=\frac{1-\lambda}{20}$ we will have:

Proposition 2.2. Suppose that $\tilde{x} \in \tilde{\Delta}_{1}, \delta \leq h \eta_{l} / 2$ and $N \in U_{\tilde{x}}^{\gamma, \delta, h}$.

- $f(N) \cap R(\tilde{f}(\tilde{x}), h) \in U_{\tilde{f}(\tilde{x})}^{\lambda \gamma, \delta\left(\frac{1+\lambda}{2}\right), h}$;

- for any two points $y_{1}, y_{2} \in N$;

$$
d_{\tilde{f}(\tilde{x})}^{\prime}\left(f\left(y_{1}\right), f\left(y_{2}\right)\right)>\left(\frac{1}{2 \lambda}+\frac{1}{2}\right) d_{\tilde{x}}^{\prime}\left(y_{1}, y_{2}\right)
$$

Proof. The proof will be similar as the proof of proposition 2.4 of [K1].

Lemma 2.3. For any $l>1, \beta<1 / 4,0<h \leq 1$ there exists a number $\alpha=\alpha(\chi, l, \beta, h)$ 
s.t. if $\tilde{x}, \tilde{y} \in \tilde{\Delta}_{l}$ and $\tilde{d}(\tilde{x}, \tilde{y}) \leq \alpha, N \in U_{\tilde{y}}^{4 \beta \gamma, h \beta \eta_{l}, h} \operatorname{resp} .\left(N \in S_{\tilde{y}}^{4 \beta \gamma, h \beta \eta_{l}, h}\right)$ then $N$ is an admissible $(u, h, \tilde{x})$-manifold (resp. $(s, h, \tilde{x})$-manifold) near the point $x$.

Proof. Fix a small Pesin block $\tilde{\Delta}_{l}$ for some $l>1$ such that $\tilde{x}, \tilde{y} \in \tilde{\Delta}_{l}$. Using properties of Pesin blocks and 2.1 we see that Lyapunov charts depend continuously on $\tilde{x}$ over $\tilde{\Delta}_{1}$. When $\tilde{d}(\tilde{x}, \tilde{y}) \leq \alpha$ then by definition of admissible manifolds we see that $N$ not only is an admissible $(u, h, \tilde{y})$-manifold (resp. $(s, h, \tilde{y})$-manifold) near the point $y$ but also an admissible $(u, h, \tilde{x})$-manifold (resp. $(s, h, \tilde{x})$-manifold) near the point $x$.

Proposition 2.4. Let $\tilde{x} \in \tilde{\Delta}_{l}, 0<h \leq 1$. Then any admissible $(s, h, \tilde{x})$-manifold near the point $x$ intersects any admissible $(u, h, \tilde{x})$-manifold near to $x$ at exactly one point and the intersection is transversal.

Proof. - (i) Existence: Let $K=\Phi_{\tilde{x}}(\operatorname{graph}(\psi)), L=\Phi_{\tilde{x}}(\operatorname{graph}(\bar{\psi}))$ be an admissible $(s, h, \tilde{x})$ - manifold and an admissible $(u, h, \tilde{x})$ - manifold near $x$ respectively, such that:

$$
\psi \in C^{1}\left(B^{s}\left(h \eta_{l} / 2\right), B^{u}\left(h \eta_{l} / 2\right)\right) \quad \text { and } \quad \bar{\psi} \in C^{1}\left(B^{u}\left(h \eta_{l} / 2\right), B^{s}\left(h \eta_{l} / 2\right)\right) .
$$

Let us consider the map $\left.\left.\bar{\psi} \circ \psi: B\left(h \eta_{l} / 2\right)\right) \rightarrow B\left(h \eta_{l} / 2\right)\right)$. Since this map is continuous it has a fixed point $u_{0}$ (by the Brower fixed point theorem). Thus, $\bar{\psi}\left(\psi\left(u_{0}\right)\right)=u_{0}$ or:

$$
\left(u_{0}, \psi\left(u_{0}\right)\right)=\left(\bar{\psi}\left(\psi\left(u_{0}\right)\right), \psi\left(u_{0}\right)\right) .
$$

But $\left(u_{0}, \psi\left(u_{0}\right)\right) \in \operatorname{graph} \psi$ and $\left(\bar{\psi}\left(\psi\left(u_{0}\right), \psi\left(u_{0}\right)\right) \in \operatorname{graph}(\bar{\psi})\right.$ therefore 1.14 implies that:

$$
\Phi_{\tilde{x}}\left(u_{0}, \psi\left(u_{0}\right)\right) \in K \cap L
$$

(ii) Uniqueness. Let $\left(u_{0}, v_{0}\right) \in \operatorname{graph} \psi \cap \operatorname{graph}(\bar{\psi})$. If $(u, v) \in \operatorname{graph} \psi$ then following inequalities hold:

$$
\begin{gathered}
\left\|v-v_{0}\right\| \leq \gamma\left\|u-u_{0}\right\| \\
\left\|v-v_{0}\right\| \geq \gamma^{-1}\left\|u-u_{0}\right\| .
\end{gathered}
$$

Since $\gamma<1$, the inequalities 2.12 and 2.13 are satisfied simultaneously only for $u=u_{0}$, $v=v_{0}$.

(iii) Transversality. Once more let $\left(u_{0}, v_{0}\right) \in \operatorname{graph}(\psi) \cap \operatorname{graph}(\bar{\psi})$. If $\theta=\left(\theta_{1}, \theta_{2}\right) \in$ $T_{\left(u_{0}, v_{0}\right)} \operatorname{graph}(\psi)$ then: 


$$
\left\|\theta_{2}\right\| \leq \gamma\left\|\theta_{1}\right\|
$$

If $\theta=\left(\theta_{1}, \theta_{2}\right) \in T_{\left(u_{0}, v_{0}\right)} \operatorname{graph}(\bar{\psi})$ then:

$$
\left\|\theta_{2}\right\| \geq \gamma^{-1}\left\|\theta_{1}\right\|
$$

Thus if $\theta \in T_{\left(u_{0}, v_{0}\right)} \operatorname{graph}(\psi) \cap T_{\left(u_{0}, v_{0}\right)} \operatorname{graph}(\bar{\psi})$ then by 2.13 and 2.14 we have $\theta=0$. This means that the intersection is transversal.

\subsection{Katok closing lemma for Endomorphisms}

Let $\mu$ be an ergodic invariant measure for an endomorphism $f: M \rightarrow M$ with non-zero Lyapunov exponents.

Lemma 2.5. (Main Lemma-) Let $f$ be a $C^{2}$ endomorphism of a compact Riemannian surface $M$. For any positive numbers $l, \delta$ there exists a number $\varrho=\varrho(l, \delta)>0$ such that if for some point $\tilde{x} \in \tilde{\Delta}_{l}$ (Pesin block) and some integer $m$ one has

$$
\tilde{f}^{m}(\tilde{x}) \in \tilde{\Delta}_{l} \quad \text { and } \quad \tilde{d}\left(\tilde{x}, \tilde{f}^{m}(\tilde{x})\right)<\varrho,
$$

then there exists a point $z \in M$ and $\bar{z} \in M^{f}$ such that $z=\pi(\bar{z})$ and

- $f^{m}(z)=z$ and $\tilde{f}^{m}(\bar{z})=\bar{z}$;

- $d_{m}^{f}(x, z)<\delta ;\left(d_{m}^{f}\right.$ is defined as $d_{m}^{f}(x, z)=\max _{0 \leq i \leq m-1} d\left(f^{i} x, f^{i} z\right)$.)

- the point $z$ is a hyperbolic periodic point for $f$ and its $W_{l o c}^{s}(x)$ and $W_{l o c}^{u}(\bar{z})$ manifolds are admissible manifolds near the point $x$ respectively.

Let fix some number $\beta>\frac{1+\lambda(\chi)}{10}$, and for some $0<h \leq 1$ assume that $\tilde{d}\left(\tilde{x}, \tilde{f}^{m}(\tilde{x})\right)<\alpha$. Where $\alpha=\alpha(\chi, l, \beta, h)$ comes from lemma 2.3. Remember that for a Pesin block, the Lyapunov metrics depend continuously on $\tilde{x}$. Then for any $0<\tau<1$, we can find the $\varrho$ in a way that for $\forall \tilde{y}_{1}, \tilde{y}_{2} \in R(\tilde{x}, h)$ :

$$
\tau<\frac{d_{\tilde{x}}^{\prime}\left(\tilde{y}_{1}, \tilde{y}_{2}\right)}{d_{\tilde{f}^{m}(\tilde{x})}^{\prime}\left(\tilde{y}_{1}, \tilde{y}_{2}\right)}<\tau^{-1} .
$$


Proof. The proof of closing lemma for an endomorphism of a compact connected surface $M$ in general is similar to the diffeomorphism case. Using the same method as in Katok's paper summarizing some details, we try to give the general idea of the proof in 3 steps.

- Step 1: Define $\Phi_{\tilde{x}}(0)=x$ and denote by $A_{0}$ and $B_{0}$ the following manifolds:

$$
A_{0}=\Phi_{\tilde{f}^{m}(\tilde{x})}\left(B_{h \eta_{l}} \times\{0\}\right) \in S_{\tilde{f}^{m}(\tilde{x})}^{0,0, h}, \quad B_{0}=\Phi_{\tilde{x}}\left(\{0\} \times B_{h \eta_{l}}\right) \in U_{\tilde{x}}^{0,0, h}
$$

$A_{0}$ and $B_{0}$ are respectively admissible $\left(\mathrm{s}, \mathrm{h}, \tilde{f}^{m}(\tilde{x})\right)$ and $(\mathrm{u}, \mathrm{h}, \tilde{x})$ manifolds near $x$ (Lemma 2.3). Notice that they can be extended to some $\tilde{B}_{0} \in U_{\tilde{x}}^{\gamma, 0, h}$ and $\tilde{A}_{0} \in \in$ $S_{\tilde{x}}^{\gamma, 0, h}$ for some small $\gamma>0$ and we continue denoting them by $A_{0}$ and $B_{0}$.

Lets choose some Lyapunov charts made through the forward orbit of $\tilde{x}$ and backward orbit of $\tilde{f}^{m}(\tilde{x})$ lets define the manifolds $A_{0}^{i}, B_{0}^{i}$ for $i=1,2, \ldots, m-1$ as:

$$
\begin{aligned}
& \left\{\begin{aligned}
A_{0}^{1} & =f_{\tilde{f}^{m}(\tilde{x})}^{-1}\left(A_{0}\right) \cap R\left(\tilde{f}^{m-1}(\tilde{x}), h\right), \\
A_{0}^{2} & =f_{\tilde{f}^{m-1}(\tilde{x})}^{-1}\left(A_{0}^{1}\right) \cap R\left(\tilde{f}^{m-2}(\tilde{x}), h\right), \\
\vdots & \\
A_{0}^{i}= & f_{\tilde{f}^{m-i-1}(\tilde{x})}^{-1}\left(A_{0}^{i-1}\right) \cap R\left(\tilde{f}^{m-i}(\tilde{x}), h\right), \text { for } i=1,2, \ldots, m-1
\end{aligned}\right. \\
& \left\{\begin{array}{r}
B_{0}^{1}=f\left(B_{0} \cap R(\tilde{x}, h)\right) \\
B_{0}^{2}=f\left(B_{0}^{1} \cap R(\tilde{f}(\tilde{x}), h)\right) \\
\vdots \\
B_{0}^{i}=f\left(B_{0}^{i-1} \cap R\left(\tilde{f}^{i-1}(\tilde{x}), h\right)\right), \text { for } i=1,2, \ldots, m-1
\end{array}\right.
\end{aligned}
$$

And let

$$
\begin{aligned}
& A_{1}=f_{\tilde{f}^{-1}(\tilde{x})}^{-1}\left(A_{0}^{m-1}\right) \cap R(\tilde{x}, h) \\
& B_{1}=f\left(B_{0}^{m-1}\right) \cap R(\tilde{x}, h)
\end{aligned}
$$

Notice that $f^{-1} A_{0}^{i}$ may have different pre-images. We take the one which has intersection with chosen Lyapunov chart and to be more clear, in context we are denoting it as $f_{\tilde{f}^{m}(\tilde{x})}^{-1}$. Moreover again by Lemma 2.3 when $d\left(x, f^{m}(x)\right)$ is small enough then $A_{1}$ and $B_{1}$ become admissible manifolds near $x$. (figure 2.3)

Now using induction we can define $A_{n}, A_{n}^{1}, \ldots, A_{n}^{m-1}, B_{n}, B_{n}^{1}, \ldots, B_{n}^{m-1}, n=1,2, \ldots$ in the following way 


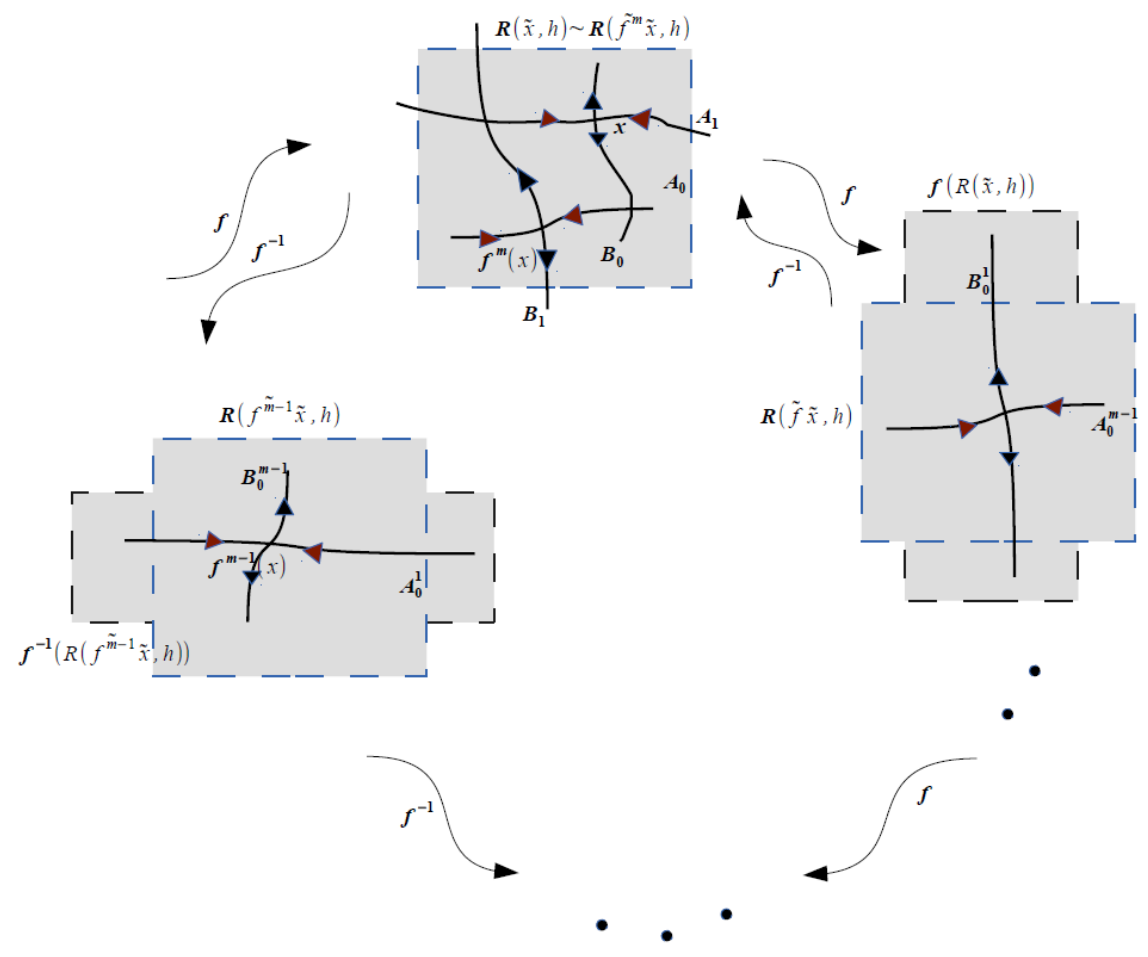

Figure 2.3

$$
\begin{aligned}
& \begin{cases}A_{n}^{1} & =f_{\tilde{f}^{m}(\tilde{x})}^{-1}\left(A_{n}\right) \cap R\left(\tilde{f}^{m-1}(\tilde{x}), h\right), \\
A_{n}^{i} & =f_{\tilde{f}^{m-i-1}(\tilde{x})}^{-1}\left(A_{n}^{i-1}\right) \cap R\left(\tilde{f}^{m-i}(\tilde{x}), h\right), \\
\vdots & \\
A_{n+1} & =f_{\tilde{f}^{-1}(\tilde{x})}^{-1} A_{n}^{m-1} \cap R(\tilde{x}, h), \quad i=2, \ldots, m-1\end{cases} \\
& \begin{cases}B_{n}^{1}=f\left(B_{n} \cap R(\tilde{x}, h)\right) \\
B_{n}^{i}=f\left(B_{n}^{i-1} \cap R\left(\tilde{f}^{i-1}(\tilde{x}), h\right)\right), \quad i=2, . ., m-1 \\
\vdots \\
B_{n+1}=f\left(B_{n}^{m-1}\right) \cap R(\tilde{x}, h)\end{cases}
\end{aligned}
$$

$A_{m}, B_{m}$ are respectively admissible stable and unstable manifolds near to $x$. By Proposition 2.4 we know that every admissible $(s, h, \tilde{x})$-manifold $A_{k}, k \geq 1$ near the point $x$, intersects any other admissible $(u, h, \tilde{x})$-manifold $B_{l}, l \geq 0$ near $x$ in exactly one point and their intersection is transversal. We denote this points of transversal intersections by $z_{k, l}$. Obviously $x=z_{10}$ and $f^{m}(x)=z_{01}$. (Figure2.3)

Lemma 2.6. If $k \geq 1, l \geq 0$ then $f^{m}\left(z_{k, l}\right)=z_{k-1, l+1}$. 
Proof. We need to show that

1. $f^{m} z_{k, l} \in A_{k-1}$

2. $f^{m} z_{k, l} \in B_{l+1}$

By definition $z_{k, l}=A_{k} \cap B_{l}$ which means $z_{k, l} \in A_{k}$, that implies $f^{m} z_{k, l} \in f^{m} A_{k} \subset$ $f^{m-1} A_{k-1}^{m-1} \subset \ldots \subset A_{k-1}$. For 2 , it is enough to show that for $i=1, \ldots, m-1$ :

$$
f^{i} z_{k, l} \subset B_{l}^{i}
$$

If this happens then $f^{m-1} z_{k, l} \subset B_{l}^{m-1}$ and $f^{m} z_{k, l} \subset f\left(f^{m-1} z_{k, l}\right) \subset f\left(B_{l}^{m-1}\right)$. But since $f^{m} z_{k, l} \subset A_{k-1} \subset R(\tilde{x}, h)$ then $f^{m} z_{k, l} \in B_{l+1}=f\left(B_{l}^{m-1}\right) \cap R(\tilde{x}, l)$. To see that $f^{i} z_{k, l} \subset B_{l}^{i}$, by induction on $i$, suppose $f^{i-1} z_{k, l} \in B_{l}^{i-1}$ and use the definition 2.20 .

Assume we choose $\tau$ in 2.16 to be

$$
\tau>(1 / 2+1 / 2 \lambda)^{-1 / 100}
$$

$\left(\lambda=\lambda(\chi)\right.$ is the same as, in the proposition 2.1.) For every $k_{1}, k_{2} \geq 1, l \geq 0$ :

$$
d_{\tilde{x}}^{\prime}\left(z_{k_{1}, l}, z_{k_{2}, l}\right) \leq \lambda^{\prime} d_{\tilde{x}}^{\prime}\left(z_{k_{1}-1, l+1}, z_{k_{2}-1, l+1}\right)
$$

where $\lambda^{\prime}=\lambda^{\prime}(\chi, m)=(1 / 2+1 / 2 \lambda)^{-m+1 / 100}<1$.

Through the construction of $z_{k, l}$ we have $f^{i} z_{k, l} \in B_{l}^{i} \cap R\left(\tilde{f}^{i}(\tilde{x}), h\right)$. The $B^{l}$ is an admissible $(u, h, \tilde{x})-$ manifold near the point $x$. Remind that by Proposition 2.2, for $y_{1}, y_{2} \in U_{\tilde{x}}^{\gamma(\chi), \delta, h}$ where $\delta \leq h \eta_{l} / 2$ and $\gamma=\frac{1-\lambda}{20}$ :

$$
d_{\tilde{f}(\tilde{x})}^{\prime}\left(f\left(y_{1}\right), f\left(y_{2}\right)\right)>(1 / 2 \lambda+1 / 2) d_{\tilde{x}}^{\prime}\left(y_{1}, y_{2}\right)
$$

Now using 2.22 and lemma 2.16 and the fact that $f^{i} z_{k, l} \in B_{l}^{i} \cap R\left(\tilde{f}^{i}(\tilde{x}), h\right)$ :

$$
\begin{aligned}
d_{\tilde{x}}^{\prime}\left(z_{k_{1}-1, l+1}, z_{k_{2}-1, l+1}\right) & \geq \tau d_{\tilde{f}^{m} \tilde{x}}^{\prime}\left(z_{k_{1}-1, l+1}, z_{k_{2}-1, l+1}\right) \\
& \geq \tau(1 / 2+1 / 2 \lambda)^{m} d_{\tilde{x}}^{\prime}\left(z_{k_{1}, l}, z_{k_{2}, l}\right) \\
& \geq \hat{\lambda}^{-1} d_{\tilde{x}}^{\prime}\left(z_{k_{1}, l}, z_{k_{2}, l}\right) .
\end{aligned}
$$

In a similar way is possible to see that 2.21 works for $k \geq 0, l_{1}, l_{2} \geq 1$ and we have 
the following two inequalities:

$$
\left\{\begin{array}{l}
d_{\tilde{x}}^{\prime}\left(z_{k_{1}, l}, z_{k_{2}, l}\right) \leq \lambda^{\prime} d_{\tilde{x}}^{\prime}\left(z_{k_{1}-1, l+1}, z_{k_{2}-1, l+1}\right) \\
d_{\tilde{x}}^{\prime}\left(z_{k, l_{1}}, z_{k, l_{2}}\right) \leq \lambda^{\prime} d_{\tilde{x}}^{\prime}\left(z_{k+1, l_{1}-1}, z_{k+1, l_{2}-1}\right)
\end{array}\right.
$$

Lemma 2.7. $\lim _{k \rightarrow \infty} d_{\tilde{x}}^{\prime}\left(z_{k, k-1}, z_{k-1, k}\right)=0$.

Proof. By triangular inequality we have that

$$
d_{\tilde{x}}^{\prime}\left(z_{k, k-1}, z_{k-1, k}\right) \leq d_{\tilde{x}}^{\prime}\left(z_{k, k-1}, z_{k-1, k-1}\right)+d_{\tilde{x}}^{\prime}\left(z_{k-1, k-1}, z_{k-1, k}\right) .
$$

Let estimate each term in the right-hand part of inequality:

The points $z_{k, k-1}$ and $z_{k-1, k-1}$ belong to the admissible $(u, h, \tilde{x})$-manifold $B_{k-1}$. From lemma 2.6 we have $f^{m} z_{k, l}=z_{k-1, l+1}$, then:

$$
\left\{\begin{array}{l}
f^{m(k-1)} z_{k-1, k-1}=z_{0,2 k-2} \in B_{2 k-2} \\
f^{m(k-1)} z_{k, k-1}=z_{1,2 k-2} \in B_{2 k-2}
\end{array}\right.
$$

For every $i=0, \ldots, k-2$ by 2.23 :

$$
d_{\tilde{x}}^{\prime}\left(z_{k-1-i, k-1+i}, z_{k-i, k-1+i}\right) \leq \lambda^{\prime} d_{\tilde{x}}^{\prime}\left(z_{k-2-i, k+i}, z_{k-1-i, k+i}\right)
$$

and so:

$$
\left\{\begin{array}{l}
d_{\tilde{x}}^{\prime}\left(z_{k, k-1}, z_{k-1, k-1}\right) \leq \grave{\lambda}^{k-1} d_{\tilde{x}}^{\prime}\left(z_{1,2 k-2}, z_{0,2 k-2}\right) \leq 2 \eta_{l} h \dot{\lambda}^{k-1} \\
d_{\tilde{x}}^{\prime}\left(z_{k-1, k-1}, z_{k-1, k}\right) \leq \lambda^{k-1} d_{\tilde{x}}^{\prime}\left(z_{2 k-2,1}, z_{2 k-2,0}\right) \leq 2 \eta_{l} h \dot{\lambda}^{k-1}
\end{array}\right.
$$

Going back to 2.24 reminding that $\lambda^{\prime}<1$ implies:

$$
\lim _{k \rightarrow \infty} d_{\tilde{x}}\left(z_{k, k-1}, z_{k-1, k}\right)=0
$$

Using above lemmas, when $k \rightarrow \infty$, the two sequences $z_{k-1, k}$ and $z_{k, k-1} k=1,2, \ldots$ converge to some $z \in R(\tilde{x}, h)$. In other words

$$
f^{m} z=\lim _{k \rightarrow \infty} f^{m} z_{k, k-1}=\lim _{k \rightarrow \infty} z_{k-1, k}=\lim _{k \rightarrow \infty} z_{k, k-1}=z .
$$

Consequently we have $\bar{z}=\left(\ldots z f(z) \ldots f^{m-1}(z) z \ldots\right)$ which is periodic with the same 
period. This proves the first item of the Main Lemma.

- Step 2: Since we had $f^{i} z_{k, l} \in f^{-1}\left(B_{l}^{i+1}\right)=B_{l}^{i} \cap R\left(\tilde{f}^{i}(\tilde{x}), h\right)$ and $f$ is continuous,

$$
f^{i}(z)=\lim _{k \rightarrow \infty} f^{i} z_{k, k-1} \in R\left(\tilde{f}^{i}(\tilde{x}), h\right)
$$

Consequently (for a chosen $\mu$-ergodic measure and $\chi=\min \left|\lambda_{j}\right|, j=1,2$ as $\eta_{l}$ is considered to be a constant not depending on $\left.\tilde{x} \in \tilde{\Delta}=\bigcup_{\chi, l} \Delta_{l}(\chi, l)\right)$ taking $\eta\left(\tilde{f}^{i}(\tilde{x})\right)=r$

$$
d_{\tilde{f}^{i}(\tilde{x})}^{\prime}\left(f^{i} x, f^{i} z\right) \leq 2 h \eta_{l} \leq 2 h r .
$$

The $M$ is compact and 2.6 estimate says that exists $K=1 / 2$ thus for any $\tilde{y} \in \tilde{\Delta}_{l}$ and every two points $w_{1}, w_{2} \in R(\tilde{x}, 1)$ :

$$
d\left(w_{1}, w_{2}\right)<K^{-1} d_{\tilde{y}}^{\prime}\left(w_{1}, w_{2}\right)
$$

Therefore:

$$
d_{n}^{f}(x, z) \leq \max _{0 \leq i \leq n-1} d\left(f^{i} x, f^{i} z\right) \leq d_{\tilde{f}^{i}(\tilde{x})}^{\prime}\left(f^{i} x, f^{i} z\right) \leq 2 K^{-1} h r
$$

Now taking $h<\frac{\delta}{r}$ we obtain the second item of the Main Lemma.

- Step 3: The last step of the proof is showing the hyperbolicity of $d_{z} f^{n}$. As $z$ stays near to the Pesin block along its orbit, hence a natural conclusion is that, he picks up enough hyperbolicity, even may belong to $\Delta_{l}$. To show this fact Katok uses cone method that to avoid of prolonged calculations we shall omit the counts and may bring some parts in Appendix A.

Assuming the hyperbolicity of $d_{z} f^{n}$ (Appendix), we need to show that the local stable- unstable manifolds of $z$ are admissible manifolds. Katok shows for diffeomorphisms the case of local stable manifolds and the same can happen for stable manifolds of endomorphisms too. We bring the proof for local unstable manifolds in a $\tilde{x}$-chart view and the rest can be viewed in step 7 of [K1]. The general idea for this part comes from looking at extended $A_{k}, B_{k},(k=0,1, \ldots)$ admissible $(1, \tilde{x})$-manifolds with $\tilde{x} \in \tilde{\Delta}_{l}$. Define the following set: 


$$
\begin{aligned}
U_{\tilde{x}}: & =\left\{W_{j} \mid(u, 1, \tilde{x})-\text { admissible unstable manifolds near } x\right\} \\
& =\left\{\Phi_{\tilde{x}}\left(\operatorname{graph}\left(\psi_{i}\right)\right) \mid \psi_{i} \in C^{0}\left(B_{\eta_{l} / 2}, B_{\eta_{l} / 2}\right)\right\} .
\end{aligned}
$$

Let $W_{1}, W_{2} \in U_{\tilde{x}}$, then we can define the metric

$$
\rho\left(W_{1}, W_{2}\right)=\max _{u \in B_{\eta_{l} / 2}}\left\|\psi_{1}(u)-\psi_{2}(u)\right\|
$$

which provides a $C^{0}$ - topology with closure $\bar{U}_{\tilde{x}}$ compact. Consequently exists a subsequence $B_{n_{l}},(l=1,2, \ldots)$ of $B_{n},(n=0,1, .$.$) such that converges to some manifold$ $B \subset \overline{U_{\tilde{x}}}$ in this $C^{0}$-topology. Showing that $B$ is contained in the local unstable manifold of $\tilde{z}$ we conclude that locally near $z$ the manifold $W^{u}(\tilde{z})$ would have the following form:

$$
W_{l o c}^{u}(\tilde{z})=\Phi_{\tilde{x}}(\operatorname{graph} \psi)
$$

where $\psi \in C^{0}\left(B_{\eta_{l} / 2}, B_{\eta_{l} / 2}\right) \subset B \subset \overline{U_{\tilde{x}}}$.

Lemma 2.8. For $w \in B$ we will have

$-f_{\tilde{f}^{m}(\tilde{x})}^{-m n} w \in R(\tilde{x}, 1), n=1,2, \ldots$;

- for some constants $C>0, \hat{\lambda}<1: d\left(f_{\tilde{f}^{m}(\tilde{x})}^{-m n} w, f_{\tilde{z}}^{-m n} z\right)<C \hat{\lambda}^{m n} d(w, z)$.

Proof. Before entering in the proof of lemma, recall that $\tilde{x}$ is the recurrent point of closing lemma belonging to $\tilde{\Delta}_{1}$ and exists some integer $m$ and $\varrho=\alpha>0$ such that $\tilde{d}\left(\tilde{x}, \tilde{f}^{m}(\tilde{x})\right)<\alpha$. fixing some $n$. Exists a sequence of points $w_{l} \in B_{n_{l}}$ such that $w=\lim _{l \rightarrow \infty} w_{l}$. By 2.20 we see that:

$$
B_{n_{l}} \subset R(\tilde{x}, 1) \Rightarrow f_{\tilde{f}^{m}(\tilde{x})}^{-m n} w \in R(\tilde{x}, 1)
$$

and using 5.1, 2.6 and 2.16:

$$
\begin{aligned}
d\left(f_{\tilde{f}^{m} \tilde{x}}^{-m n} w, z\right)=d\left(f_{\tilde{f}^{m} \tilde{x}}^{-m n} w, f_{\tilde{f}^{m} \tilde{x}}^{-m n} z\right) & \leq d_{\tilde{f}^{m}(\tilde{x})}^{\prime}\left(f_{\tilde{f}^{m}(\tilde{x})}^{-m n}(w), f_{\tilde{f}^{m}(\tilde{x})}^{-m n}(z)\right) \\
& <2 \tau^{-1} d_{\tilde{x}}^{\prime}\left(f_{\tilde{f}^{m}(\tilde{x})}^{-m n}(w), f_{\tilde{f}^{m}(\tilde{x})}^{-m n}(z)\right) \\
& <2 \tau^{-1}\left(\frac{1}{2}+\frac{1}{2 \lambda}\right)^{-m n} d_{\tilde{f}^{m}(\tilde{x})}^{\prime}(w, z) \\
& <2 \tau^{-1} a_{l}\left(\frac{1}{2}+\frac{1}{2 \lambda}\right)^{-m n} d(w, z) .
\end{aligned}
$$


Taking $\hat{\lambda}=\left(\frac{1}{2 \lambda}+\frac{1}{2}\right)^{-1}<1$ and $C=2 \tau^{-1} a_{l}>0$ we reach to the second item of the lemma. This lemma specially helps to conclude that $B$ is contained in the local unstable manifold of $\bar{z}$. Therefore locally near $z$ the manifold $W^{u}(\bar{z})$ will have the following form:

$$
W_{l o c}^{u}(\bar{z})=\Phi_{\tilde{x}}(\operatorname{graph} \psi)
$$

where $\psi \in C^{0}\left(B_{\eta_{l} / 2}, B_{\eta_{l} / 2}\right) \subset B \subset \overline{U_{\tilde{x}}}$.

This ends the last step of the proof of closing lemma.

\subsection{Hyperbolic Periodic Orbits and Horseshoe}

We say that a compact $f$-invariant set $\Gamma$ is a horseshoe of $f$ if there exist positive integers $l, m$ and subsets $\Gamma_{0}, \cdots, \Gamma_{m-1}$ of $M$ such that

$$
\Gamma=\Gamma_{0} \cup \cdots \cup \Gamma_{m-1}, \quad f\left(\Gamma_{i}\right)=\Gamma_{i+1}(\bmod -m),
$$

and the inverse limit $\left.\tilde{f}^{m}\right|_{\tilde{\Gamma}_{0}}: \tilde{\Gamma}_{0} \rightarrow \tilde{\Gamma}_{0}$ of $\left.f^{m}\right|_{\Gamma_{0}}: \Gamma_{0} \rightarrow \Gamma_{0}$ is topologically conjugate to a full-shift in l-symbols. Here $\tilde{\Gamma}_{0}$ is defined as

$$
\tilde{\Gamma}_{0}=\left\{\tilde{x}=\left(x_{n}\right) \in M^{f}: x_{n} \in \Gamma_{0}, \text { for all } n \in \mathbb{Z}\right\}
$$

In a similar way as for diffeomorphisms, we can claim that the following theorems are valid for endomorphisms. Let

$$
\operatorname{Per}_{h}(f)=\{p \in \operatorname{Per}(f): p \text { is hyperbolic and has transversal homoclinic point. }\}
$$

Theorem 2.9. Let $f: M \rightarrow M$ a $C^{2}$ endomorphism of a compact Riemannian manifold. Assume that $\nu$ is a hyperbolic $f$-invariant measure, then Supp $\subset \overline{\operatorname{Per}(f)}$. Where $\operatorname{Per}(f)$ is the set of hyperbolic periodic points.

Proof. For $\nu$ a hyperbolic $f$-invariant measure, we can consider its unique $\tilde{f}$ - invariant measure $\tilde{\nu}$ on $M^{f}$ that $\pi_{*}(\tilde{\nu})=\nu$. Suppose $\tilde{y}$ be a point belonging to $\operatorname{supp} \tilde{\nu}$ and $\pi(\tilde{y})=y$. Let take $B(\tilde{y}, \epsilon)$ with $\tilde{\nu}(B(\tilde{y}, \epsilon))>0$ and choose $\tilde{\Delta}_{l}$ in a way that $\tilde{\nu}\left(B(\tilde{y}, \epsilon) \cap \tilde{\Delta}_{l}\right)>0$. Considering the $\varrho$ in closing lemma, we can choose $V \subset B(\tilde{y}, \epsilon) \cap \tilde{\Delta}_{l}$ with $\tilde{\nu}(V)>0$ and diameter less than $\varrho$. Now applying the Poincare recurrence theorem and Closing lemma, we find $\bar{z}$ a hyperbolic periodic point belonging to $V$. Once $\tilde{\nu}(B(\tilde{y}, \epsilon))>0$, then 
$\nu(B(y, \epsilon))=\tilde{\nu}\left(\pi^{-1}(\pi(B(\tilde{y}, \epsilon)))\right) \geq \tilde{\nu}(B(\tilde{y}, \epsilon))>0$ and we can conclude that the hyperbolic point $z=\pi(\bar{z}) \in B(y, \epsilon)$ and Supp $\subset \overline{\operatorname{Per}(f)}$.

Theorem 2.10. Let $\nu$ be a non-atomic hyperbolic measure. Then

- Suppv is contained in the closure of the set of hyperbolic periodic points that have transverse homoclinic points;

- if $\nu$ is ergodic then Supp $\nu$ is contained in the closure of the set of transverse homoclinic points of exactly one hyperbolic periodic point.

Proof. Fix $\tilde{x}$ a point in Supp $\tilde{\nu}$ and take $\tilde{B}(\tilde{x}, \epsilon / 4)$ in such a way that for $l>0$, then $\tilde{\nu}\left(\tilde{B}(\tilde{x}, \epsilon / 4) \cap \tilde{\Delta}_{l}\right)>0$ and $\tilde{B}(\tilde{x}, \epsilon / 4) \subset \tilde{\Delta}_{l}$. We take two distinct points $\tilde{x_{1}}, \tilde{x_{2}} \in \tilde{B}(\tilde{x}, \epsilon / 4)$. Using Poincar recurrence theorem and closing lemma, for some smaller disjoint balls around each of these points, we show that exists $\overline{z_{1}} \neq \overline{z_{2}}$ hyperbolic periodic points. By 2.5 and 2.4, exists $W^{u}\left(\bar{z}_{i}\right)$ as $(u, 1, \tilde{x})$-admissible and $W^{s}\left(\bar{z}_{i}\right)$ as $(S, 1, \tilde{x})$-admissible manifolds which intersect transversally in exactly one point. The existence of these transversal intersections can guarantee that $z_{1}, z_{2} \in M$ are transversal homoclinic points (they are the projections of $\overline{z_{1}}$ and $\overline{z_{2}}$ ). In fact once we have these transversal intersections using $\lambda$-lemma, we can show that $z_{1}, z_{2}$ are transversal homoclinic points. To prove the second statement it is enough to show that the closure of the set of transverse homoclinic points of a periodic point in $M$ is $f$-invariant. More details in theorem 15.4.3 of [BP2] or theorem 4.2 of $[\mathrm{K} 1]$.

Theorem 2.11. Let $f: M \rightarrow M$ be a $C^{2}$ endomorphism. Suppose that $f$ has a continuous non-atomic hyperbolic measure. Then there exists a hyperbolic horseshoe for $f$.

Proof. The existence of a horseshoe is an immediate consequence of last theorem. 


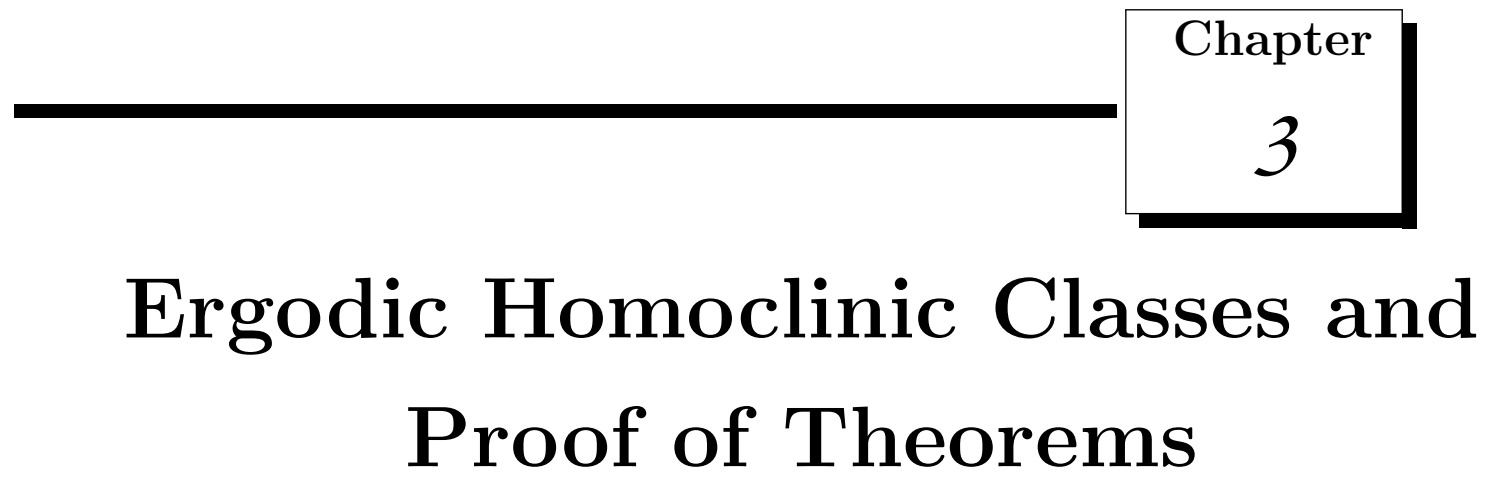

The notion of ergodic homoclinic classes comes from works of [HHTU]. F. Rodriguez Hertz, M. Rodriguez Hertz, A. Tahzibi and R. Ures, defined this notion proving the uniqueness of SRB measures for surface transitive diffeomorphisms. Here we will define them in a similar way for endomorphisms.

\subsection{Ergodic Homoclinic Classes.}

For $f: M \rightarrow M$ a $C^{2}$ non-uniformly hyperbolic endomorphism on a smooth closed Riemannian surface $M$. We can define the Ergodic Homoclinic Class $(E H C)$ for a hyperbolic periodic point $p \in M$ with period $n$, as $\Lambda(p):=\Lambda^{s}(p) \cap \Lambda^{u}(p)$, where

$$
\Lambda^{s}(p):=\left\{x \in \mathcal{R} \mid W^{s}(x) \pitchfork W^{u}(\bar{p}) \neq \emptyset\right\}
$$

and

$$
\Lambda^{u}(p):=\left\{x \in \mathcal{R} \mid W^{u}(x) \pitchfork W^{s}(O(p)) \neq \emptyset\right\} .
$$

Where $\mathcal{R}=\pi(\tilde{\mathcal{R}})$ from Oseledet's theorem and $W^{s}(O(p))=\bigcup_{i=0}^{n} W^{s}\left(f^{i}(p)\right)$. Notice that $W^{s}(x)=W^{s}(\tilde{x})$ by Observation 1.23. Similarly for $\bar{p}=\left(\ldots p_{1} p_{2} \ldots p_{n} p_{1} p_{2} \ldots\right) \in M^{f}$ we 
may define the inverse limit ergodic homoclinic class $(\widetilde{E H C})$ as $\tilde{\Lambda}(\bar{p}):=\tilde{\Lambda}^{s}(\bar{p}) \cap \tilde{\Lambda}^{u}(\bar{p})$.

$$
\tilde{\Lambda}(\bar{p}):=\left\{\tilde{x} \in \tilde{\mathcal{R}} \mid \exists n \geq 0 \text { s.t. } W^{u}(\tilde{x}) \pitchfork W^{s}(O(p)) \neq \emptyset, W^{s}\left(\tilde{f}^{n}(\tilde{x})\right) \pitchfork W^{u}(\bar{p}) \neq \emptyset\right\} .
$$

Suppose $\mu$ is a hyperbolic $f$-invariant Borel probability measure. By Theorem 1.1 exists the unique $\tilde{f}$-invariant measure $\tilde{\mu}$ on $M^{f}$ corresponding to $\mu$. Observe that by Definition $\pi(\tilde{\Lambda}(\bar{p}))=\Lambda(p)$.

Take $\tilde{x} \in \tilde{\Delta}_{l}$ a recurrent point in the support of $\tilde{\mu}$ restricted to the Pesin block $\tilde{\Delta}_{l}$. Use closing lemma to find a hyperbolic periodic point $\bar{p}$. We prove two crucial lemmas about the Ergodic homoclinic class of $\bar{p}$.

Lemma 3.1. $\tilde{\mu}(\tilde{\Lambda}(\bar{p}))>0$ and $\mu(\Lambda(p))>0$;

Let $\tilde{B}$ be a small ball around $\tilde{x}$ such that $\tilde{\mu}\left(\tilde{B} \cap \tilde{\Delta}_{l}\right)>0$. By the last item in the closing lemma $2.5, W^{u}(\bar{p})$ and $W^{s}(\bar{p})$ are respectively admissible manifolds near to $\mathrm{x}$. By continuity of stable and unstable manifolds in the Pesin blocks and using propositions 2.3,2.4, choosing $\tilde{B}$ small enough, for any point $\tilde{y} \in \tilde{B} \cap \tilde{\Delta}_{l}$, we have that the by transversality arguments $\tilde{y} \in \tilde{\Lambda}(\bar{p})$. The second claim in the lemma is clear from the definition of $\tilde{\mu}$. (see 1.1)

Lemma 3.2. $\tilde{\Lambda}(\bar{p})$ is $\tilde{f}$-invariant and $\Lambda(p)$ is forward-invariant.

We will show that $\tilde{\Lambda}(\bar{p})$ is $\tilde{f}$-invariant and the second claim is a simple consequence of definition. Firstly let us prove that $\tilde{f}(\tilde{\Lambda}(\bar{p})) \subset \tilde{\Lambda}(\bar{p})$. Recall that $\tilde{\Lambda}(\bar{p})=\tilde{\Lambda}^{s}(\bar{p}) \cap \tilde{\Lambda}^{u}(\bar{p})$ and $\Lambda(p)=\Lambda^{s}(p) \cap \Lambda^{u}(p)$. Without of loss of generality suppose that $p$ is a hyperbolic fixed point, then:

$$
\begin{aligned}
\tilde{x} \in \tilde{\Lambda}^{u}(\bar{p}) & \Rightarrow W^{u}(\tilde{x}) \pitchfork W^{s}(p) \neq \emptyset \\
& \Rightarrow f\left(W^{u}(\tilde{x})\right) \pitchfork W^{s}(p) \neq \emptyset \\
& \Rightarrow W^{u}(\tilde{f}(\tilde{x})) \pitchfork W^{s}(p) \neq \emptyset \\
& \Rightarrow \tilde{f}(\tilde{x}) \in \tilde{\Lambda}^{u}(\bar{p}) . \\
\tilde{x} \in \tilde{\Lambda}^{s}(\bar{p}) & \Rightarrow W^{s}(x) \pitchfork W^{u}(\bar{p}) \neq \emptyset \\
& \Rightarrow f\left(W^{s}(x)\right) \pitchfork W^{u}(\bar{p}) \neq \emptyset \\
& \Rightarrow W^{s}(f(x)) \pitchfork W^{u}(\bar{p}) \neq \emptyset \\
& \Rightarrow \tilde{f}(\tilde{x}) \in \tilde{\Lambda}^{s}(\bar{p}) .
\end{aligned}
$$


Now we prove that $\tilde{\Lambda}(\bar{p}) \subset \tilde{f}(\tilde{\Lambda}(\bar{p}))$. We divide the proof into two steps:

- $\tilde{\Lambda}^{u}(\bar{p}) \subset \tilde{f}\left(\tilde{\Lambda}^{u}(\bar{p})\right)$ : Take $\tilde{x}=\tilde{f}\left(\tilde{f}^{-1}(\tilde{x})\right) \in \tilde{\Lambda}^{u}(\bar{p})$. Let us verify that $\tilde{f}^{-1}(\tilde{x}) \in$ $\tilde{\Lambda}^{u}(\bar{p})$ or by definition $W^{u}\left(\tilde{f}^{-1}(\tilde{x})\right) \pitchfork W^{s}(\bar{p}) \neq \emptyset$. By contradiction suppose that $W^{u}\left(\tilde{f}^{-1}(\tilde{x})\right) \pitchfork W^{s}(\bar{p})=\emptyset$. So,

$$
\begin{aligned}
& f\left(W^{u}\left(\tilde{f}^{-1}(\tilde{x})\right)\right) \pitchfork f\left(W^{s}(\bar{p})\right)=\emptyset \\
& \Rightarrow W^{u}(\tilde{x}) \pitchfork W^{s}(\bar{p})=\emptyset .
\end{aligned}
$$

which yields a contradiction.

- $\tilde{\Lambda}^{s}(\bar{p}) \subset \tilde{f}\left(\tilde{\Lambda}^{s}(\bar{p})\right)$ : Once again taking $\tilde{x}=\tilde{f}\left(\tilde{f}^{-1}(\tilde{x})\right)$. We need to prove that $\exists n \geq 0$ such that $W^{s}\left(\tilde{f}^{n-1}(\tilde{x})\right) \pitchfork W^{u}(\bar{p}) \neq \emptyset$. By contradiction, suppose that

$$
\begin{aligned}
& W^{s}\left(\tilde{f}^{n-1}(\tilde{x})\right) \pitchfork W^{u}(\bar{p})=\emptyset \\
& \Rightarrow W^{s}\left(\tilde{f}^{n}(\tilde{x})\right) \pitchfork W^{u}(\bar{p}) \neq \emptyset .
\end{aligned}
$$

which yields a contradiction.

Finally observe that $\pi(\tilde{\Lambda}(\bar{p}))=\pi(\tilde{f}(\tilde{\Lambda}(\bar{p}))=f(\pi(\tilde{\Lambda}(\bar{p}))) \Rightarrow f(\Lambda(p))=\Lambda(p)$. Using $\lambda$ lemma we have the following result.

Lemma 3.3. If $p$ and $q$ are two homoclinically related periodic points, then $\Lambda^{u}(p)=\Lambda^{u}(q)$.

Proof. We will show that $\Lambda^{u}(p) \subset \Lambda^{u}(q)$. Let take $x \in \Lambda^{u}(p)$ and obtain that $x \in \Lambda^{u}(q)$ and vise-versa. For simplicity we suppose that $p$ and $q$ are hyperbolic fixed points. As $x \in \Lambda^{u}(p)$ means for some $\tilde{x} \in \tilde{\mathcal{R}}, W^{u}(\tilde{x}) \pitchfork W^{s}(p) \neq \emptyset$. To say that $x \in \Lambda^{u}(q)$, we need to show $W^{u}(x) \pitchfork W^{s}(q) \neq \emptyset$. For this purpose we use $\lambda$-lemma for a small disc around $W^{u}(\tilde{x}) \pitchfork W^{s}(p) \neq \emptyset$. Then exists some large $\mathrm{k}$ that makes some part of $f^{k}\left(W^{u}(\tilde{x})\right)$ sufficiently $C^{1}$ close to $W^{u}(\bar{p})$ in a way that using $p \sim q$,

$$
f^{k} W^{u}(\tilde{x}) \pitchfork W^{s}(q) \neq \emptyset
$$

Observe that by definition $W^{u}\left(\tilde{f}^{k}(\tilde{x})\right)=f^{k}\left(W^{u}(\tilde{x})\right)$ and $f^{k}\left(W^{s}(q)\right)=W^{s}(q), \forall k \in \mathbb{Z}^{+}$. Now we claim that also happens $W^{u}(\tilde{x}) \pitchfork W^{s}(q) \neq \emptyset$. that is because if not, $W^{u}(\tilde{x}) \pitchfork$ $W^{s}(q)=\emptyset$, and $f^{k}\left(W^{u}(\tilde{x})\right) \pitchfork f^{k} W^{s}(q)=\emptyset$ which contradicts the 3.3.

the same process can be done for showing $\Lambda^{u}(q) \subset \Lambda^{u}(p)$, and therefore $\Lambda^{u}(p)=\Lambda^{u}(q)$ and the proof ends. 


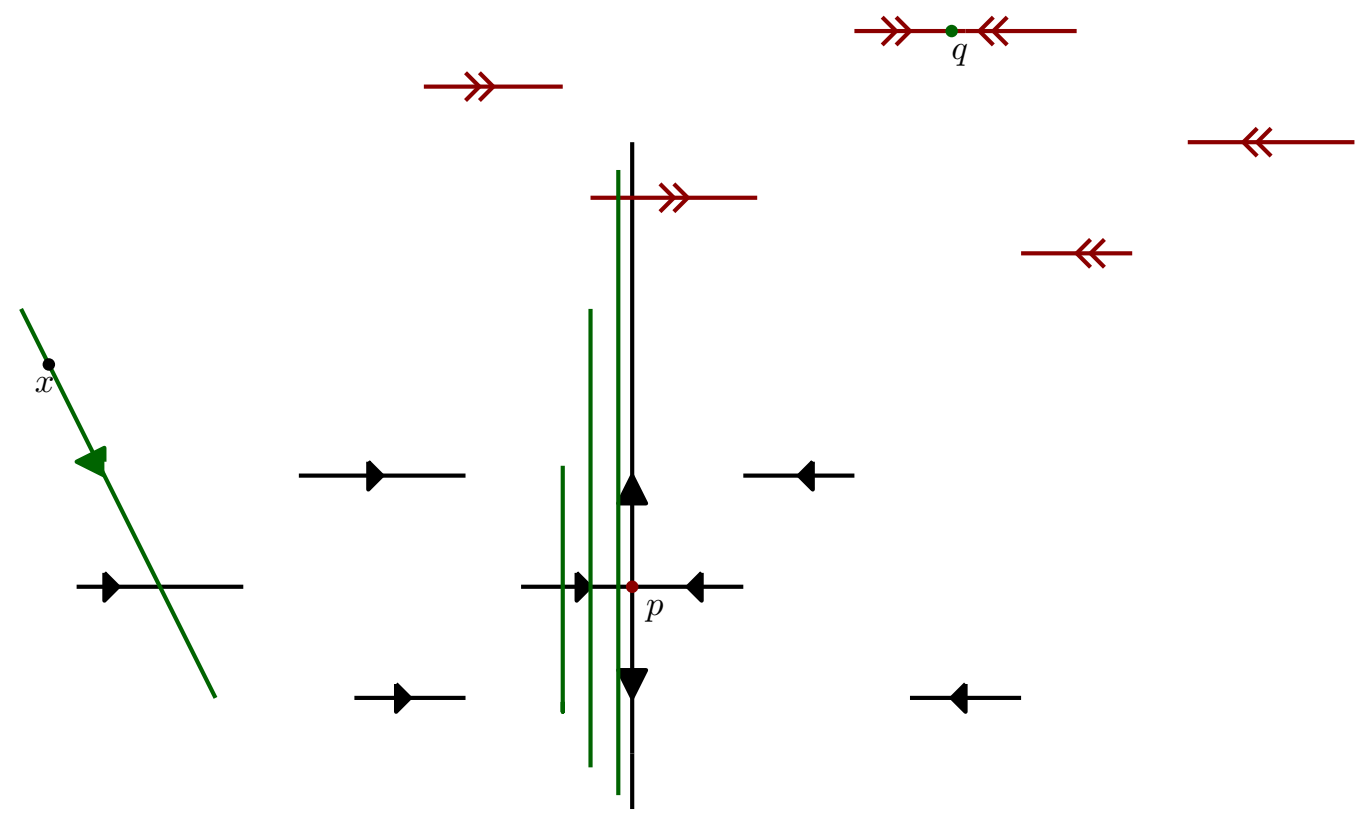

Figure 3.1: Lambda-lemma

\subsection{Ergodic Criterion.}

In this section we are going to show that if $\Lambda(p)$ for $p$ "some hyperbolic periodic point" has positive measure, then it coincide with a hyperbolic ergodic component which contains p. Before giving the main theorem of this part we need to remember once again that $f$ is not invertible necessarily and this may do not give enough information through Birkhoff Ergodic Theorem on $M$. For this reason we will write the inverse limit version of BET on natural extension of system $(M, f)$.

Definition 3.4 (Density Point). Let $(X, d)$ a metric space and $\mu$ any Borel probability measure and $B_{\epsilon}(x)$ is a ball around $x$ for any small $\epsilon>0$ (in metric topology). We call $x$ the density point of a subset $A \in \mathcal{B}(X)$ if:

$$
\lim _{\epsilon \rightarrow 0} \frac{\mu\left(B_{\epsilon}(x) \cap A\right)}{\mu\left(B_{\epsilon}(x)\right)}=1 .
$$

Theorem 3.5 (Birkhoff Theorem for Natural Extension[M4]). Let $\tilde{f}: M^{f} \rightarrow M^{f}$ be the lift homeomorphism on inverse limit space of $f$ and $\tilde{\mu}$ the unique $\tilde{f}$-invariant lift measure of a Borel probability $f$-invariant measure $\mu$ on $M$. Let $\tilde{\phi} \in C\left(M^{f}\right)$ a continues function on $M^{f}$. For $\tilde{\mu}$ almost every point $\tilde{x} \in M^{f}$ we have $\lim \frac{1}{n} \sum_{j=0}^{n-1} \tilde{\phi}\left(\tilde{f}^{j}(\tilde{x})\right)$ exists and goes to some $\bar{\phi}(\tilde{x})$ that is a $\tilde{\mu}$ - integrable function with $\bar{\phi} \circ \tilde{f}=\bar{\phi}$ and moreover $\int \bar{\phi} d \tilde{\mu}=\int \tilde{\phi} d \tilde{\mu}$. In particular if $\mu$ is ergodic on $M$, then $\bar{\phi}$ is constant $\tilde{\mu}$.a.e. 
Observation 3.6. By Birkhoff theorem on Natural Extension which we may denote it as $\widetilde{B E T}$, we see that for any given $\tilde{f}$-invariant $\tilde{\mu}$ as above, and $\tilde{\phi} \in C\left(M^{f}\right)$,

$$
\bar{\phi}^{ \pm}(\tilde{x})=\lim _{n \rightarrow \pm \infty} \frac{1}{n} \sum_{j=0}^{n-1} \tilde{\phi}\left(\tilde{f}^{j}(\tilde{x})\right)
$$

$\tilde{\mu}$-a.e. exists and moreover almost every point $\tilde{x} \in M^{f}, \bar{\phi}^{ \pm}(x)$ is $\tilde{f}$-invariant and $\bar{\phi}^{+}(\tilde{x})=$ $\bar{\phi}^{-}(\tilde{x})$.

Lemma 3.7 (Typical points for continuous functions). There exists an invariant set $\tilde{J}$ of typical points with $\tilde{\mu}(\tilde{J})=1$ such that for all $\tilde{\phi} \in C\left(M^{f}\right)$, if $\tilde{x} \in \tilde{J}$ then for all $\tilde{\omega} \in \widetilde{W^{s}}(\tilde{x})$ and $\tilde{\mu}$ a.e. $\tilde{\omega} \in \widetilde{W^{u}}(\tilde{x})$,

$$
\bar{\phi}^{+}(\tilde{x})=\bar{\phi}^{+}(\tilde{\omega})
$$

Proof. Let us consider the following full measure set of typical points:

$$
\tilde{S}_{0}=\left\{\tilde{x} \in M^{f}: \bar{\phi}^{+} \text {and } \bar{\phi}^{-} \text {are well - defined at } \tilde{x} \text { and } \bar{\phi}^{+}=\bar{\phi}^{-}\right\} \text {. }
$$

Recall that $\tilde{\mu}$ almost everywhere point in $\tilde{S}_{0}$ is regular. For almost all $\tilde{x} \in \tilde{S}_{0}$, we have that $\tilde{\mu}_{\tilde{x}}^{u}$ almost everywhere $\tilde{\zeta} \in \widetilde{W_{l o c}^{u}}(\tilde{x})$ then $\tilde{\zeta} \in \tilde{S}_{0}$. Since if not, then would exist a $\tilde{\mu}$-positive measure $\tilde{A} \subset M^{f}$ of points that are not typical. To see more precisely, look at $A=\pi(\tilde{A})$, the projection of $\tilde{A}$ on $M$. It will have some $\mu$-positive measure subset $B$ that the elements do not belong to $S_{0}=\pi\left(\tilde{S}_{0}\right)$. In fact for every such $x \in A$, there is a subset $B_{x} \subset W_{l o c}^{u}(\tilde{x}) \backslash S_{0}$ with $\mu_{\tilde{x}}^{u}\left(B_{x}\right)>0$ that considering a density point $y$ of $A$ and integrating along a transverse small disk $T$, we obtain $B \subset M \backslash S_{0}$ that

$$
\mu(B)=\int_{T} \mu_{x}^{u}\left(B_{x}\right) d \mu_{T}>0
$$

This is an absurd because firstly the existence of such $B$ contradicts with $\mu\left(S_{0}\right)=1$ and secondly if you look at the pre-image of this set, by definition $\tilde{\mu}\left(\pi^{-1}(B)\right)=\mu(B)>0$ and $\pi^{-1}(B) \cap \tilde{S}_{0}=\emptyset$. Which contradicts with $\widetilde{B E T}$ too.

Now let

$$
\tilde{S}_{1}=\left\{\tilde{x} \in \tilde{S}_{0}: \tilde{\mu}_{\tilde{x}}^{u}-\text { almost everywhere } \tilde{\zeta}, \in \widetilde{W^{u}}(\tilde{x}), \tilde{\zeta} \in \tilde{S}_{0}\right\}
$$

For all $\tilde{x}, \tilde{\zeta} \in \tilde{S}_{1}$, we have

$$
\bar{\phi}^{+}(\tilde{x})=\bar{\phi}^{-}(\tilde{x}) \quad \text { and } \quad \bar{\phi}^{+}(\tilde{\zeta})=\bar{\phi}^{-}(\tilde{\zeta})
$$

On the other hand from continuity of $\tilde{\phi}$ we know that $\bar{\phi}^{-}(\tilde{\zeta})=\bar{\phi}^{-}(\tilde{x})$ for all $\left.\tilde{\zeta} \in \widetilde{W^{u}}(\tilde{x})\right\}$. 
This plus 3.4 conclude that $\bar{\phi}^{+}(\tilde{\zeta})=\bar{\phi}^{+}(\tilde{x})$. As $\tilde{S}_{1}$ is an $\tilde{f}$-invariant set,( by $\widetilde{B E T}$, $\bar{\phi}$ is $\tilde{f}$-invariant) we take $\tilde{J}=\tilde{S}_{1}$. Notice that $\tilde{\phi}$ is a continuous function and using the definition of stable sets, $\bar{\phi}^{+}$becomes constant on $\widetilde{W_{l o c}^{s}}(\tilde{x})$.

Lemma 3.8. Given $\tilde{\phi} \in \mathcal{L}^{1}\left(M^{f}\right)$, there exists an invariant set $\tilde{S}_{\tilde{\phi}} \subset M^{f}$ with $\tilde{\mu}\left(\tilde{S}_{\tilde{\phi}}\right)=1$ such that if $\tilde{x} \in \tilde{S}_{\tilde{\phi}}$ then $\tilde{\mu}_{\tilde{x}}^{u}-$ a.e $\tilde{y} \in \widetilde{W}^{u}(\tilde{x})$ satisfies

$$
\bar{\phi}^{+}(\tilde{y})=\bar{\phi}^{+}(\tilde{x})
$$

Proof. Given $\tilde{\phi} \in \mathcal{L}^{1}\left(M^{f}\right)$, as $C\left(M^{f}\right)$ is dense in $\mathcal{L}^{1}\left(M^{f}\right)$ therefore we can take a sequence of continuous functions $\tilde{\phi}_{n}$ converging to $\tilde{\phi}$. By $\widetilde{B E T}, \tilde{\mu}$-a.e $\tilde{x}, \bar{\phi}_{n}^{+}(\tilde{x})$ exists and $\bar{\phi}_{n}^{+}(\tilde{x})$ converges to $\bar{\phi}^{+}(\tilde{x})$. As $M^{f}$ is a compact metric space so exists a subsequence $\bar{\phi}_{n_{k}}^{+}$such that $\tilde{\mu}$-a.e $\tilde{x}$ converges to $\bar{\phi}^{+}(\tilde{x})$. The intersection of this set of almost everywhere convergence with the set $\tilde{S}_{1}$ of lemma 3.7 for every of $\bar{\phi}_{n_{k}}^{+}$gives us the desired $\tilde{S}_{\tilde{\phi}}$.

Following two theorems play important roles in this chapter.

Theorem 3.9. Let $f: M \rightarrow M a C^{2}$ endomorphism over a compact manifold $M$ equipped with a hyperbolic measure $\mu$ with $S R B$ property. So if $\tilde{\mu}(\tilde{\Lambda}(\bar{p}))>0$, then

$$
\tilde{\Lambda}^{u}(\bar{p}) \subset^{\circ} \tilde{\Lambda}^{s}(\bar{p})
$$

Proof. First remind that by definition $\tilde{\Lambda}(\bar{p}):=\tilde{\Lambda}^{u}(\bar{p}) \cap \tilde{\Lambda}^{s}(\bar{p})$ where

$$
\begin{aligned}
& \tilde{\Lambda}^{u}(\bar{p}):=\left\{\tilde{x} \in \tilde{\mathcal{R}} \mid \pi(\tilde{x})=x, W^{u}(\tilde{x}) \pitchfork W^{s}(p) \neq \emptyset\right\} \\
& \tilde{\Lambda}^{s}(\bar{p}):=\left\{\tilde{x} \in \tilde{\mathcal{R}} \mid \pi(\tilde{x})=x, \exists n \geq 0, \text { s.t } W^{s}\left(\tilde{f}^{n}(\tilde{x})\right) \pitchfork W^{u}(\bar{p}) \neq \emptyset\right\} .
\end{aligned}
$$

To show $\tilde{\Lambda}^{u}(\bar{p}) \subset^{\circ} \tilde{\Lambda}^{s}(\bar{p}) \tilde{\mu}$ - a.e. It is enough to show that $\tilde{x} \in \tilde{\Lambda}^{u}(\bar{p}) \cap \tilde{S}_{\tilde{\Lambda}^{s}}$ implies $\tilde{x} \in \tilde{\Lambda}^{s}(\bar{p})$.

Let take $\tilde{y} \in \tilde{\Lambda}^{s}(\bar{p})$ an auxiliary point in a way that for $l>0$ both $\tilde{x}, \tilde{y}$ lies in the same pesin block $\tilde{\Delta}_{l}$ and $\tilde{y} \in \operatorname{supp}\left(\left.\tilde{\mu}\right|_{\tilde{\Delta}_{l} \cap \tilde{\Lambda}^{s}(\bar{p})}\right)$. As $\tilde{y} \in \tilde{\Lambda}^{s}(\bar{p})$ then by definition $W^{s}(y) \pitchfork W^{u}(\bar{p}) \neq \emptyset$. Take $s \in W^{s}(y) \pitchfork W^{u}(\bar{p}) \neq \emptyset$ then $d\left(f^{n}(s), f^{n}(y)\right) \stackrel{n \rightarrow \infty}{\longrightarrow} 0$. It says that for large enough $n$ and some $\delta>0$ small, the $d\left(W^{u}(\bar{p}), f^{n}(y)\right)<\delta / 2$. Let continue the proof using following two small lemmas.

Lemma 3.10. If exists a $\tilde{\mu}_{\tilde{x}}^{u}$-positive measure subset, of $\widetilde{W}^{u}(\tilde{x})$ belonging to $\tilde{\Lambda}^{s}(\bar{p})$, then $\tilde{x} \in \tilde{\Lambda}^{s}(\bar{p})$. 
Proof. The $\tilde{\Lambda}^{s}(\bar{p})$ is $\tilde{f}$-invariant and $1_{\tilde{\Lambda}^{s}(\bar{p})}=1_{\tilde{\Lambda}^{s}(\bar{p})} \circ \tilde{f}$. This implies that if $\tilde{x} \notin \tilde{\Lambda}^{s}(\bar{p})$ then $\tilde{\mu}_{\tilde{x}}^{u}-$ a.e. $\tilde{y} \in \widetilde{W}_{\text {loc }}^{u}(\tilde{x})$ does not belong to $\tilde{\Lambda}^{s}(\bar{p})$.

Lemma 3.11. If exists some $m \in \mathbb{N}$ such that a $\tilde{\mu}_{\tilde{f}^{m}(\tilde{x})}^{u}$-positive measure subset, of $\widetilde{W}^{u}\left(\tilde{f}^{m}(\tilde{x})\right)$ belong to $\tilde{\Lambda}^{s}(\bar{p})$, then $\tilde{x} \in \tilde{\Lambda}^{s}(\bar{p})$.

Proof. This comes from the fact that $\tilde{x} \in \tilde{S}_{\tilde{\tilde{\Lambda}}^{s}}$ and $\tilde{S}_{1_{\tilde{\Lambda}} s}$ is an $\tilde{f}$-invariant set. The rest will be a corollary of last lemma.

Now let continue the proof of the theorem 3.9. Recall $f^{n}(y)$ from beginning part of proof, using Poincaré recurrence theorem, let choose $\mathrm{n}$ in a way that $\tilde{f}^{n}(\tilde{y}) \in \tilde{\Delta}_{l}$ and put $\alpha:=f^{n}(y)$. By definition $x \in \Lambda^{u}(p)$ and $W^{u}(\tilde{x}) \pitchfork W^{s}(p) \neq \emptyset$ and $\alpha$ is such that $W_{\delta}^{s}(\alpha) \pitchfork W^{u}(\bar{p}) \neq \emptyset$. Using $\lambda$ - lemma we find some large $\mathrm{m}$ that $f^{m}(x) \in \Delta_{l}$ and

$$
W^{u}\left(\tilde{f}^{m}(\tilde{x})\right) \pitchfork W_{\delta}^{s}(\alpha) \neq \emptyset
$$

By hypothesis $\mu$ is hyperbolic with SRB property and $\pi_{*}\left(\tilde{\mu}_{\tilde{x}}^{u}\right) \ll m_{\tilde{x}}^{u}$. Let call $\pi_{*}\left(\tilde{\mu}_{\tilde{x}}^{u}\right)=$ $\mu_{\tilde{x}}^{u}$. The conditional measures are similar (continuous) with respect to $m_{\tilde{x}}^{u}$, the Lebesgue measure on $W_{\tilde{x}}^{u}$. We want to find a positive $\tilde{\mu}_{\tilde{x}}^{u}-$ subset of $\widetilde{W}^{u}(\tilde{x})$ such that belongs to $\tilde{\Lambda}^{s}(\bar{p})$. Using lemma 3.11 it is enough to find it on local unstable manifold of some iterate of $x$ on $M$ and then looking at its pre-image on $M^{f}$.
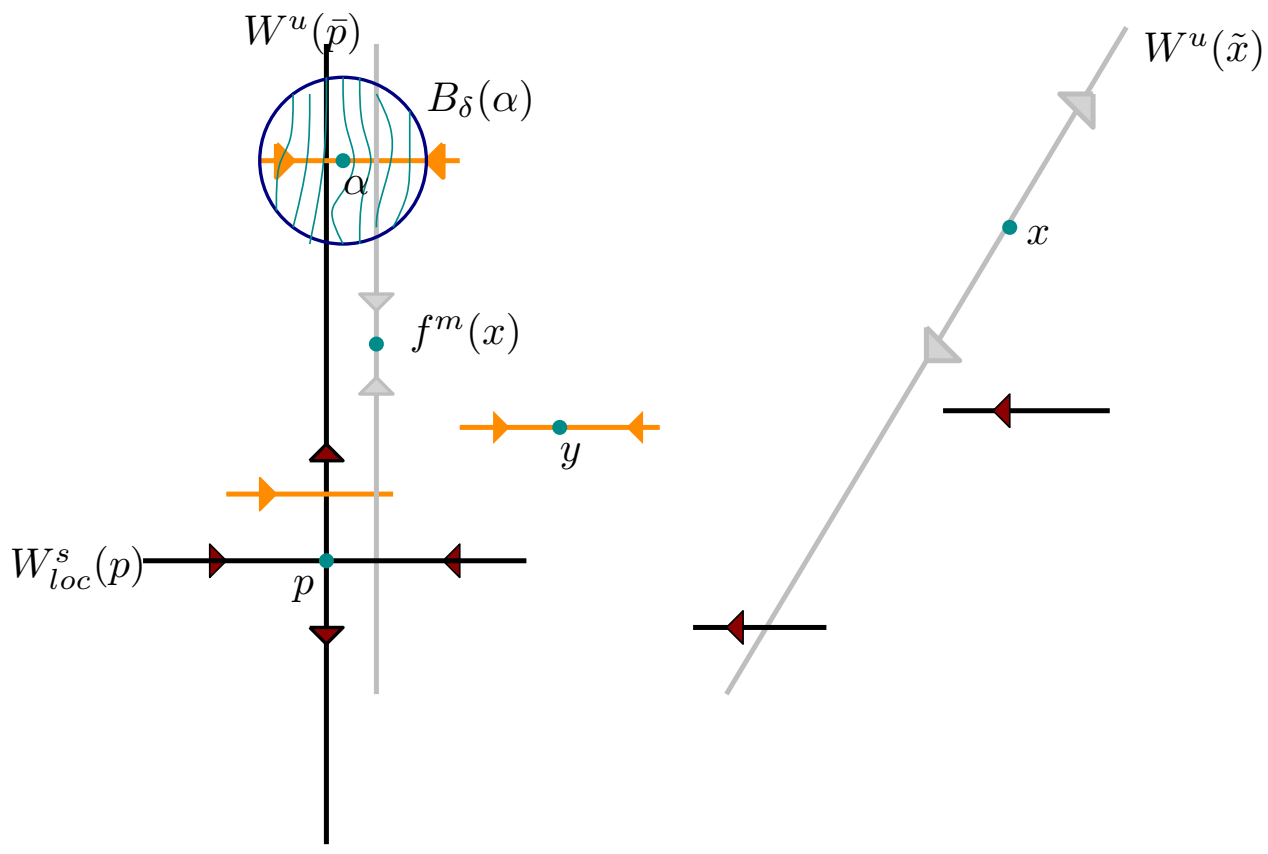

Figure 3.2: Ergodic criterion 
For this purpose, remind that $\tilde{y} \in \operatorname{Supp}\left(\left.\tilde{\mu}\right|_{\tilde{\Delta}_{l} \cap \tilde{\Lambda}^{s}(\bar{p})}\right)$ and $\alpha$ is a point of $\Delta_{l}=\pi\left(\tilde{\Delta}_{l}\right)$. Consider the ball $\tilde{B}_{\delta}(\tilde{\alpha})$ around $\tilde{\alpha}$. Covering $\tilde{B}_{\delta}(\tilde{\alpha})$ with measurable sub-ordinate partition with respect to $W^{u}$, exists some density point $\tilde{z} \in \tilde{\Delta}_{l}$ such that looking at $\widetilde{W}^{u}(\tilde{z})$, the $\tilde{\mu}_{\tilde{z}}^{u}\left(\left.\tilde{S}\right|_{\tilde{\Lambda}^{s}(\bar{p})} \cap \tilde{\Delta}_{l} \cap \tilde{B}_{\delta}(\tilde{\alpha})\right)>0$. We call this set $\tilde{Z}$. The $\tilde{Z} \subset \widetilde{W}_{\text {loc }}^{u}(\tilde{z})$ and projects on $W^{u}(\tilde{z}) \cap B_{\delta}(\alpha)$ which contains $z=\pi(\tilde{z})$ where $B_{\delta}(\alpha):=\pi\left(\tilde{B}_{\delta}(\tilde{\alpha})\right)$. Looking at the local stable foliation passing through the points belong to $\mathrm{Z}$, we have

$$
m_{\tilde{f}^{m}(\tilde{x})}^{u}\left(Z^{*}\right)>0 \Rightarrow \mu_{\tilde{f}^{m}(\tilde{x})}^{u}\left(Z^{*}\right)>0 .
$$

Where $Z^{*}$ is the set obtained on $W^{u}\left(\tilde{f}^{m}(\tilde{x})\right)$ through stable holonomy and by definition

$$
\tilde{\mu}_{\tilde{f}^{m}(\tilde{x})}^{u}\left(\pi^{-1}\left(Z^{*}\right)\right)=\mu_{\tilde{f}^{m}(\tilde{x})}^{u}\left(Z^{*}\right)>0 .
$$

Which completes the proof and says that $\tilde{x} \in \tilde{\Lambda}^{s}(\bar{p})$.

Corollary 3.12. An immediate conclusion of the above theorem will be that

$$
\Lambda^{u}(p) \subset^{\circ} \Lambda^{s}(p)
$$

Theorem 3.13. Let $f: M \rightarrow M$ be a $C^{2}$ endomorphism over a compact manifold $M$ and $\mu$ any measure with $S R B$ property. If $\tilde{\mu}(\tilde{\Lambda}(\bar{p}))>0$ for a hyperbolic periodic point $\bar{p}$, then $\left.\tilde{\mu}\right|_{\tilde{\Lambda}(\bar{p})}$ is an ergodic component of $\tilde{\mu}$.

Proof. For simplicity once again let assume that $\bar{p}$ is a hyperbolic fixed point. As $\tilde{\mu}(\tilde{\Lambda}(\bar{p}))>0$, taking any $\tilde{f}$ - invariant continuous function $\tilde{\phi}: M^{f} \rightarrow \mathbb{R}$, we show that $\tilde{\mu}$-a.e. points in $\tilde{\Lambda}(\bar{p}) \cap \tilde{J}\left(\tilde{J}\right.$ is the set of typical points from 3.7) are $\bar{\phi}^{+}$-constant. Which implies the ergodicity of $\left.\tilde{\mu}\right|_{\tilde{\Lambda}(\bar{p})}$. Let choose arbitrary $\tilde{x}, \tilde{y} \in \Delta_{l} \cap \tilde{\Lambda}(\bar{p}) \cap \tilde{S}_{1}:=\tilde{\Gamma}$ for some $l>0$. Without loose of generality we may assume that such $\tilde{x}, \tilde{y}$ are in the support of $\left.\tilde{\mu}\right|_{\tilde{\Lambda}(\bar{p})}$. Using Poincar recurrence theorem these points come back infinitely many times to $\tilde{\Gamma}$.

Table 3.1: Optimal information

\begin{tabular}{c|cc}
$\in$ & $\tilde{x}$ & $\tilde{y}$ \\
\hline$\tilde{\Lambda}^{s}(\bar{p})$ & $W^{s}(x) \pitchfork W^{u}(\bar{p}) \neq \emptyset$ & $W^{s}(y) \pitchfork W^{u}(\bar{p}) \neq \emptyset$ \\
\hline$\tilde{\Lambda}^{u}(\bar{p})$ & $W^{u}(\tilde{x}) \pitchfork W^{s}(p) \neq \emptyset$ & $W^{u}(\tilde{y}) \pitchfork W^{s}(p) \neq \emptyset$ \\
\hline$\tilde{J}=\tilde{S}_{1}$ & $\mu_{\tilde{x}}^{u}$.a.e. $\omega \in W_{l o c}^{u}(\tilde{x}) \Rightarrow \hat{\phi}^{+}(x)=\hat{\phi}^{+}(\omega)$ & $\mu_{\tilde{y}}^{u}$.a.e. $\omega \in W_{l o c}^{u}(\tilde{y}) \Rightarrow \hat{\phi}^{+}(y)=\hat{\phi}^{+}(\omega)$
\end{tabular}

Recall the definition of $\widetilde{E H C}$ as visible in table 3.1. Then 
- $\tilde{y} \in \tilde{\Lambda}^{s}(\bar{p})$ and for some large iterate $n$, it happens that $\tilde{f}^{n}(\tilde{y}) \in \tilde{\Gamma}$ as well as $W_{\delta}^{s}\left(f^{n}(y)\right) \pitchfork W^{u}(\bar{p}) \neq \emptyset$ for some $\delta>0$ small.

- $\tilde{x} \in \tilde{\Lambda}^{u}(\bar{p})$ and using $\lambda$-lemma similar to the proof of last theorem we can find some iterate $m$ of $\tilde{x}$ such that belongs to $\tilde{\Gamma}$ and $W^{u}\left(\tilde{f}^{m}(\tilde{x})\right)$ is $C^{1}$ enough close to $W^{u}(\bar{p})$.(Let it be $\delta / 2$ close.)

Again considering the table 3.1 Let $\tilde{B}_{\delta}\left(\tilde{f}^{n}(\tilde{y})\right)$ be a small ball around point $\tilde{f}^{n}(\tilde{y}) \in \tilde{\Gamma}$ and cover the ball by a measurable partition sub-ordinate with respect to $W^{u}$. ( $\mu$ is a measure with SRB property.) Looking at $B_{\delta}\left(f^{n}(y)\right)$ the projection of $\tilde{B}$, it is covered by parts of unstable manifolds containing $f^{m}(x)$ and $f^{n}(y)$. Considering $\tilde{\mu}$-a.e elements of Pesin blocks as density points, we can find some $\tilde{z} \in \tilde{B}_{\delta}\left(\tilde{f}^{n}(\tilde{y})\right) \cap \tilde{\Gamma}$ that $\tilde{\mu}_{\tilde{z}}^{u}$-a.e. $\tilde{\eta} \in \widetilde{W}^{u}(\tilde{z}) \Rightarrow \bar{\phi}^{+}(\tilde{z})=\bar{\phi}^{+}(\tilde{\eta})$. Let show the set of all such $\tilde{\eta}$, with $\tilde{\mathcal{Z}}$. Behold that

$$
\mu_{\tilde{z}}^{u}(\mathcal{Z})=\tilde{\mu}_{\tilde{z}}^{u}\left(\pi^{-1}(\pi(\mathcal{Z}))\right) \geq \tilde{\mu}_{\tilde{z}}^{u}(\tilde{\mathcal{Z}})>0, \quad Z=\pi \tilde{\mathcal{Z}}
$$

The $\mathcal{Z}$ becomes a subset of $W_{l o c}^{u}(\tilde{z}) \cap B_{\delta}\left(f^{n}(y)\right)$ and using local stable lamination

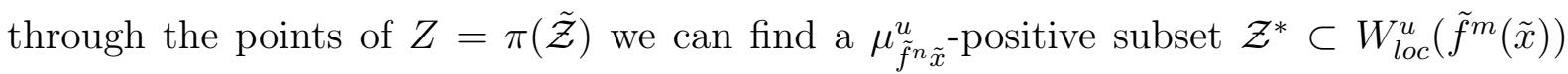
that $\hat{\phi}^{+}\left(f^{m} x\right)=\hat{\phi}^{+}(z)=$ cte for $z \in \mathcal{Z}$. Now $\tilde{\mu}_{\tilde{f}^{m}(\tilde{x})}^{u}\left(\pi^{-1}\left(\mathcal{Z}^{*}\right)\right)=\mu_{\tilde{f}^{n} \tilde{x}}^{u}\left(\mathcal{Z}^{*}\right)>0$ and as they belong to $\widetilde{W^{s}}(\tilde{z}), \forall \tilde{z} \in \tilde{\mathcal{Z}}$, then by Lemma 3.7 and 3.11 and considering a similar process on $W_{l o c}^{u}\left(\tilde{f}^{n}(\tilde{y})\right)$, we can confirm that $\bar{\phi}^{+}(\tilde{x})=\bar{\phi}^{+}(\tilde{y})=$ cte for $\tilde{\mu}$-a.e. $\tilde{x} \in \tilde{\Gamma}$. This proves that $\left.\tilde{\mu}\right|_{\tilde{\Lambda}(\bar{p})}$ is an ergodic component.

Observation 3.14. In above theorem $\hat{\phi}$ is supposed to be Birkhoff forward sum for the $f$-invariant $\phi(x)=\tilde{\phi}[\tilde{x}]$. Where $\tilde{x} \in \pi^{-1}(x)$ and $[x]$ contains all the pre-images projecting on $x$. The $\phi$ in this way is well defined and it is possible to show that by $\tilde{f}$ - invariance of $\tilde{\phi}$ then

$$
\forall \tilde{y} \in[\tilde{x}] \Rightarrow \tilde{\phi}(\tilde{y})=\tilde{\phi}(\tilde{x})
$$

\subsection{Proof of Theorems}

The proves of theorems $\mathrm{B}$ and $\mathrm{C}$ are somehow similar to the last step of the proof of the main theorem. First we bring the proof of main theorem and after that in last two subsections the proves of theorems B and C are accessible. 


\subsubsection{Proof of the Main Theorem}

Following the method of [HHTU] and using above implements we see that ergodic homoclinic classes are in a close relationship with ergodic components of a measure. In fact for any two hyperbolic ergodic measure $\mu$ and $\nu$ with SRB property, we show that they are supported on the ergodic homoclinic class of some periodic point showing them respectively with $p_{\mu}$ and $p_{\nu}$ and once the periodic points are in the same homoclinic equivalent class, then the measures become the same. Briefly we can say that the strategy of the proof consists of three steps:

- Finding $\bar{p}_{\tilde{\mu}}$ and $\bar{p}_{\tilde{\nu}}$;

- $\tilde{\Lambda}\left(\bar{p}_{\mu}\right)=\tilde{\Lambda}\left(\bar{p}_{\nu}\right)$;

- $\mu$ and $\nu$ are the same.

Theorem 3.15 (Main Theorem A.). Let $f: M \rightarrow M$ be a $C^{2}$ endomorphism over a closed Riemannian surface $M$. Then,

$\sharp\{$ Ergodic Hyperbolic Measures with SRB Property $\} \leq \sharp\{$ Homoclinic Equivalent Classes. $\}$

Proof. Let remind the definition of $p \sim q$ from definition 1.30 and take $[p]$ the homoclinic equivalent class of $p$. For every $p_{\mu}, q_{\nu} \in[p]$, (periodic points corresponding to ergodic measures $\mu$ and $\nu$ ) they are in a transversal homoclinical relation. Then proposition 3.16 and theorem 3.18 will guarantee the intersection of the basin of two measures supported on $\Lambda\left(p_{\mu}\right)$ and $\Lambda\left(q_{\nu}\right)$. This, proves the equality of measures up to homoclinic equivalent classes.

Proposition 3.16. If $p$ and $q$ are two homoclinically related periodic points, then $\Lambda(p)=$ $\Lambda(q)$.

Proof. Lemma 3.3 plus theorem 3.9 implies the proposition.

Theorem 3.17. $f: M \rightarrow M a C^{2}$ endomorphism over a compact manifold $M$ equipped with a hyperbolic measure $\mu$ with SRB property. Then for any ergodic component $\nu$ of it, there exists a hyperbolic periodic point $p$ such that $\nu(\Lambda(p))=1$.

Proof. Suppose that $\nu$ is an ergodic component of a hyperbolic measure $\mu$ with SRB property. In proposition 1.39 we have seen that ergodic components are also hyperbolic with SRB property. By Theorem 1.1 and lemma 1.3 we conclude the existence of the 
unique ergodic $\tilde{\nu}$ push-forwarding on $\nu$. In section 3.1 we observed that $\tilde{\mu}(\tilde{\Lambda}(\bar{p}))>0$ and we have shown that $\tilde{\Lambda}(\bar{p})$ is $\tilde{f}$ - invariant. They all together dispatches $\tilde{\nu}(\tilde{\Lambda}(\bar{p}))=1$ and consequently $\nu(\Lambda(p))=1$.

Theorem 3.18. Let $\mu$ and $\nu$ be ergodic measures with $S R B$ properties such that $\tilde{\mu}(\tilde{\Lambda}(\bar{p}))=$ $\tilde{\nu}(\tilde{\Lambda}(\bar{p}))=1$ for some hyperbolic periodic points $p$, then $\mu=\nu$.

Proof. Let $B(\tilde{\mu})$ and $B(\tilde{\nu})$ be respectively the basins of $\tilde{\mu}$ and $\tilde{\nu}$, where they are the inverse limit measures corresponding to $\mu$ and $\nu$.

$$
\begin{array}{ll}
B(\tilde{\mu})=\left\{\tilde{x}: \lim _{n \rightarrow \infty} \frac{1}{n} \sum_{0}^{n-1} \tilde{\phi}\left(\tilde{f}^{i}(\tilde{x})\right)=\int \tilde{\phi} d \tilde{\mu} \quad \forall \tilde{\phi} \in C\left(M^{f}\right)\right\} ; \\
B(\tilde{\nu})=\left\{\tilde{x}: \lim _{n \rightarrow \infty} \frac{1}{n} \sum_{0}^{n-1} \tilde{\phi}\left(\tilde{f}^{i}(\tilde{x})\right)=\int \tilde{\phi} d \tilde{\nu} \quad \forall \tilde{\phi} \in C\left(M^{f}\right)\right\} ;
\end{array}
$$

By hypothesis $\tilde{\mu}(\tilde{\Lambda}(\bar{p}))=\tilde{\nu}(\tilde{\Lambda}(\bar{p}))=1$ and by $\widetilde{B E T} 3.5$, we can define $B_{\tilde{\mu}}$ and $B_{\tilde{\nu}}$ such that $\tilde{\mu}\left(B_{\tilde{\mu}}\right)=\tilde{\nu}\left(B_{\tilde{\mu}}\right)=1$. They are as following:

$$
\begin{aligned}
& B_{\tilde{\mu}}=\left\{\tilde{x}: \lim _{n \rightarrow \pm \infty} \frac{1}{n} \sum_{0}^{n-1} \tilde{\phi}\left(\tilde{f}^{i}(\tilde{x})\right)=\int \tilde{\phi} d \tilde{\mu} \quad \forall \tilde{\phi} \in C\left(M^{f}\right)\right\} ; \\
& B_{\tilde{\nu}}=\left\{\tilde{x}: \lim _{n \rightarrow \pm \infty} \frac{1}{n} \sum_{0}^{n-1} \tilde{\phi}\left(\tilde{f}^{i}(\tilde{x})\right)=\int \tilde{\phi} d \tilde{\nu} \quad \forall \tilde{\phi} \in C\left(M^{f}\right)\right\} ;
\end{aligned}
$$

It means $\tilde{\mu}$ (resp. $\tilde{\nu}$ )-a.e. point $\tilde{x} \in \tilde{\Lambda}(\bar{p})$ belongs to $B_{\tilde{\mu}}$ (resp. $B_{\tilde{\nu}}$ ). If we show that $B(\mu) \cap B(\nu) \neq \emptyset$ or equivalently $B_{\mu} \cap B_{\nu} \neq \emptyset$ then we are done. (Two ergodic measures that their basin has intersection, are the same.)

Let cover the $\tilde{\Lambda}(\bar{p}) \cap B_{\tilde{\mu}}$ with sub-ordinate partition with respect to unstable manifolds. Take $\tilde{x} \in \tilde{\Lambda}(\bar{p})$ a point which $\tilde{\mu}_{\tilde{x}}^{u}\left(B_{\tilde{\mu}} \cap \tilde{\Lambda}(\bar{p})\right)=1$. By SRB property of $\mu$ and remark 1.37 we will have $m_{\tilde{x}}^{u}\left(B_{\mu} \cap \Lambda(p)\right)=1$ where $B_{\mu}:=\pi\left(B_{\tilde{\mu}}\right)$.

There exists some $\tilde{\Delta}_{l}, l \geq 1$ a Pesin block for $\left.\tilde{\nu}\right|_{\tilde{\Lambda}(\bar{p})}$ near $\bar{p}$ that considering $\tilde{y} \in \tilde{\Delta}_{l} \cap \tilde{\Delta}(\bar{p})$ a $\tilde{\nu}$ - density point of $B_{\tilde{\nu}} \cap B_{\delta}(\bar{p})$, then $m_{\tilde{y}}^{u}\left(B_{\nu} \cap \Delta_{l}\right)>0$. Here $B_{\nu}:=\pi\left(B_{\tilde{\nu}}\right), \Delta_{l}=\pi\left(\tilde{\Delta}_{l}\right)$. By Poincaré recurrence theorem $\tilde{y}$ returns back infinitely many times to $\tilde{\Delta}_{l} \cap B_{\tilde{\nu}}$ and consequently $y$ to $\Delta_{l} \cap B_{\nu}$. Moreover $\tilde{y} \in \tilde{\Lambda}(\bar{p})$, so exists some large iterate $\mathrm{n}$ that $\tilde{\alpha}=\tilde{f}^{n}(\tilde{y})$ such that $W^{u}(\tilde{\alpha})$ becomes $\delta / 2$-close to $W^{u}(\bar{p})$ in a similar way that has been explained in ergodic criteria section. Figure 3.2 
From the other side $\tilde{x} \in \tilde{\Lambda}(\bar{p})$ and $x$ belongs to $\Lambda^{u}(p)$. Using $\lambda$-lemma we may find some large iterate of $\tilde{x}$ (call it $\mathrm{m}$ ) such that $W^{u}\left(\tilde{f}^{m}(\tilde{x})\right)$ becomes near enough to $W^{u}(\bar{p})$ in a way that for a positive $\nu_{\tilde{\alpha}}^{u}$-measure $z \in \Delta_{l} \cap B_{\nu}$ then $W_{\delta}^{s}(z) \pitchfork W^{u}\left(\tilde{f}^{m}(\tilde{x})\right) \neq \emptyset$.

The stable lamination on a Pesin block is absolutely continuous [QXZ] and it implies that $m_{\tilde{f}^{m}(\tilde{x})}^{u}\left(B_{\nu} \cap \Delta_{l}\right)>0$. Also $m_{\tilde{f}^{m}(\tilde{x})}^{u}\left(B_{\mu}\right)=1$ which both together implies $B_{\mu} \cap B_{\nu} \neq \emptyset$ which finishes the proof.

Theorem 3.18 and proposition 3.16 assert the proof of main theorem and demons trates that the number of the measures with SRB properties are equal with the number of homoclinic equivalent classes where $\tilde{\Lambda}\left(\bar{p}_{\tilde{\mu}}\right)=\tilde{\Lambda}\left(\bar{q}_{\tilde{\nu}}\right)$.

\subsubsection{Proof of Theorem B}

We see that for non-uniformly expanding endomorphisms similar to the diffeomorphisms case [HHTU], it occurs the uniqueness of the measure with SRB property.

Theorem 3.19 (Theorem B.). For $f: M \rightarrow M$ a topologically transitive $C^{2}$ endomorphism over a closed Riemannian surface $M$, there exists at most one non-uniformly expanding measure with SRB property.

Proof. Let $(f, \mu)$ and $(f, \nu)$ be two $C^{2}$ topologically transitive endomorphism of nonuniformly expanding type and $\mu, \nu$ two ergodic $f$-invariant Borel probability measures with SRB property. By definition a topologically transitive map has this property that for any two open sets $U, V \subset M$ exists some $n \in \mathbb{N}$ that $f^{n} U \cap V \neq \emptyset$. For any continuous map $\phi: M \rightarrow \mathbb{R}$ let $B(\mu)$ and $B(\nu)$ be the basin of measures $\mu$ and $\nu$.

By Birkhoff Ergodic theorem for continuous map 3.5 we have $\mu(B(\mu))=1$ and $\nu(B(\nu))=1$. From the other side we have $\tilde{\mu}$ and $\tilde{\nu}$ the corresponded ergodic measures on the inverse limit space 1.1,?? which by $\widetilde{B E T}$ their basin also become with total measure.

By Proposition 1.33 there exists a measurable partition sub-ordinate to $W^{u}$ on $M^{f}$. Let consider this partition and disintegrate the $\tilde{\mu}$ along the partition elements. $\tilde{\mu}_{\tilde{x}}^{u}$ will have full conditional measures on $\widetilde{W}_{l o c}^{u}(\tilde{x})$. By SRB property of $\mu$ and observation 1.37 we can claim that $m\left(\left[W_{l o c}^{u}(\tilde{x}) \cap B(\mu)\right]^{c}\right)=0$. As $W_{l o c}^{u}(\tilde{x})$ is an open subset of $M$, let take $U=W_{l o c}^{u}(\tilde{x})$. Using the same process from $\nu$, we can obtain another open set $V$ such that $m\left([V \cap B(\nu)]^{c}\right)=0$. 
Now by transitivity of $f$, there exists some $n \in \mathbb{N}$ such that $f^{n}(U) \cap V \neq \emptyset$ and by the fact that $f$ is a local diffeomorphism, we obtain

$$
m\left(f^{n}(U \cap B(\mu)) \cap(V \cap B(\nu))\right)>0 .
$$

Therefore $B(\mu) \cap B(\nu) \neq \emptyset$ and this yields the unity of measures $\mu$ and $\nu$.

\subsubsection{Proof of Theorem C}

In this section we try to give a simple definition of partially hyperbolic endomorphisms and then giving the idea of the proof of the following theorem.

Definition 3.20. [T1] We call a $C^{2} \operatorname{map} f: M \rightarrow M$ a partially hyperbolic endomorphism if there are positive constants $\lambda$ and $C$ also a continuous decomposition of the tangent bundle $T M=E^{c u} \oplus E^{s}$ with $\operatorname{dim} E^{c u}=\operatorname{dim} E^{s}=1$, such that

- $\left\|\left.d f^{n}\right|_{E^{s}}(z)\right\|<e^{(\lambda n-c)}$ and

- $\left\|\left.d f^{n}\right|_{E^{c} u}(z)\right\|<e^{(-\lambda n+c)}\left\|\left.d f^{n}\right|_{E^{s}}(z)\right\|$

for all $z \in M$ and $n \geq 0$. The sub-bundles $E^{c u}$ and $E^{s}$ are called the central and stable sub-bundle, respectively.

Theorem 3.21 (Theorem C). Let $f: M \rightarrow M$ be a topologically transitive $C^{2}$ partially hyperbolic endomorphism with a continuous decomposition of the tangent bundle $T M=$ $E^{s} \oplus E^{c u}$. Then there exists at most one hyperbolic measure with SRB property.

Proof. To prove the uniqueness of measure for this case, suppose that $\mu, \nu$ are two ergodic measures with SRB property. As it is known, their corresponded measures $\tilde{\mu}$ and $\tilde{\nu}$ are ergodic and by $\widetilde{B E T}$ we have $\tilde{\mu}(\tilde{\mathcal{R}} \cap B(\tilde{\mu}))=1$. Let take a measurable partition subordinate to $W^{u}$ from 1.33. By disintegration along this partition, there exist some $\widetilde{W}_{l o c}^{u}(\tilde{x})$ for some $\tilde{x} \in \tilde{\mathcal{R}} \cap B(\tilde{\mu})$ such that for $\tilde{\mu}$-a.e. $\tilde{y} \in \widetilde{W}_{l o c}^{u}(\tilde{x})$, we have $\tilde{y} \in \tilde{B}(\tilde{\mu}) \cap \tilde{R}$.

After projecting to the ambient manifold, we obtain $\pi_{*} \tilde{\mu}_{\tilde{x}}^{u}\left(B(\mu) \cap \widetilde{W}_{l o c}^{u}(\tilde{x})\right)=1$. Now by Remark 1.37 we have $m_{\tilde{x}}^{u}\left(B(\mu)^{c}\right)=0$. Consider $U_{\mu}:=\bigcup_{z \in W_{l o c}^{u}(\tilde{x})} W_{l o c}^{s}(z)$. By continuity of stable foliation (which exist in the partially hyperbolic case, using Perron-Hadamard technique [HPSh]) $U_{\mu}$ is almost open. This means that $U_{\mu}$ differs from an open set in a Lebesgue zero subset. 
Using the same process we obtain $U_{\nu}$ corresponding to measure $\nu$. As $f$ is topologically transitive and local diffeomorphism, then exists some $n \in \mathbb{N}$ such that

$$
m\left(f^{n}\left(U_{\mu}\right) \cap U_{\nu}\right)>0 \Rightarrow m(B(\mu) \cap B(\nu))>0
$$

Which means $B(\mu) \cap B(\nu) \neq \emptyset$. 


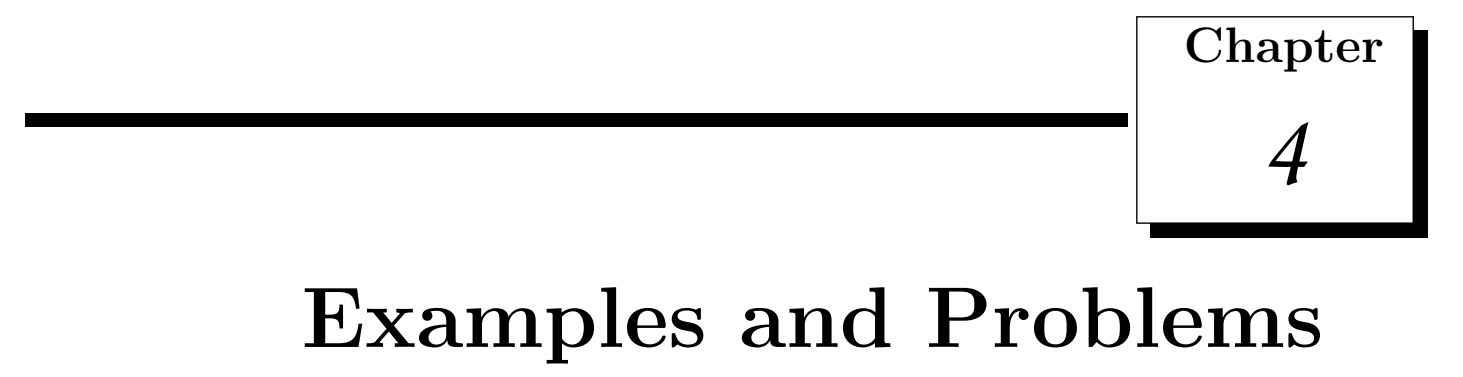

Diffeomorphisms are simple example of endomorphisms where by results od [HHTU] it happens the uniqueness of SRB measures. Examples of non-uniformly hyperbolic systems with non-zero Lyapunov exponents that theorem applies on them, are Anosov endomorphisms with $\Omega(f)=M$, where $\Omega(f)$ is the non-wandering set and $M$ the ambient closed Riemannian manifold. For such topologically transitive examples $[\mathrm{P}]$, using theorem $\mathrm{C}$ we can show that the measures with SRB property becomes unique. M. Urbanski and C. Wolf in [UW] also show the uniqueness of measures with SRB property for Axiom A, including Anosov endomorphisms.

There are also Kan type examples due to I. Kan [K3], constructed on a cylinder. It supports two homoclinical equivalent classes indeed two measures with SRB property. It would be interesting to find more examples of non-uniformly hyperbolic dynamics with non-zero Lyapunov exponents that are not uniformly hyperbolic or a diffeomorphism. In what follows we bring some examples which we believe the theorems are applicable. Example I is some explanation on Kan type examples. Example II is using Katok's "Slow down method" from [BP2] to construct a non- uniformly hyperbolic endomorphism in the lack of a zero measure set of points with Lebesgue zero Lyapunov exponents. Example III is some part of the Sumi's article $[\mathrm{S}]$ on constructing a "derived from Anosov" endomorphism with one source, and example IV is taken from [CV] about non- uniformly expandings. 


\subsection{Example I}

Consider $F: S^{1} \times[0,1] \rightarrow S^{1} \times[0,1]$ of the form $F(x, t)=\left(k x \bmod \mathbb{Z}, f_{x}(t)\right)$ where $k$ is some integer number with $|k| \geq 3$. Then $x \longmapsto k x \bmod \mathbb{Z}$ has at least two fixed points denoted $p$ and $q$. Assume $F$ is $C^{2}$ and

- $F$ preserves the boundary components: $f_{x}(0)=0$ and $f_{x}(1)=1$;

- $f_{p}$ and $f_{q}$ has exactly two fixed points each, a source at $t=1$ (respectively $t=0$ ) and a sink at $t=0$ (respectively $t=1)$;

- $\left|d_{x} f(t)\right|<3$ at every point $(x, t) \in S^{1} \times[0,1]$;

- $\int \log d_{x} f(0) d x<0$ and $\int \log d_{x} f(1) d x<0$.

Let also have a look at Kan's original example:

$$
F(x, t)=(3 t, x+x(1-x) \cos (2 \pi t) / 32) .
$$

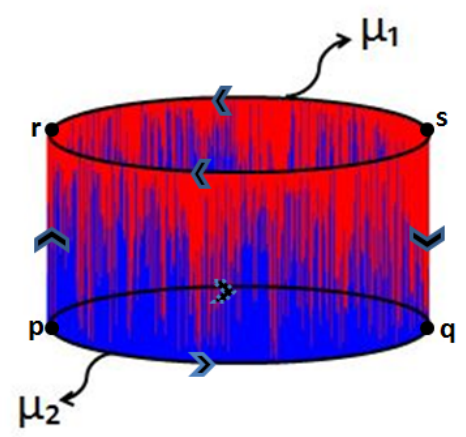

Figure 4.1: The Kan example with two intermingled basins, colored red and blue.[M3]

As we see this type of examples are constructed boundary preserving and with at least two fixed points in each boundary region. Let take $\mu_{1}$ and $\mu_{2}$ correspondingly the Lebesgue measures restricted to top and bottom boundaries. Let call the top boundary $\partial_{1}$ and the bottom $\partial_{2}$. Then $\mu_{1}=\left.m\right|_{\partial_{1}}$ and $\mu_{2}=\left.m\right|_{\partial_{2}}$. It is not difficult to see that both $\mu_{1}$ and $\mu_{2}$ are $F$-invariant probabilities. Item four of the general definition for Kan type examples can guarantee that the chosen measures are hyperbolic too. Ina private communication M. Anderson and J. Yang mentioned that, it may be possible to construct such transitive examples on $T^{2}$ with two SRB measures. The idea is gluing two such Kan 
type examples in a subtle way.[A] We emphasize that, if the construction is possible then it shows that the estimate in our main theorem is optimal.

An invariant probability measure is called a Physical Measure if its basin has positive Lebesgue measure. In the case of ergodic hyperbolic measures it is possible to show that the physical measures and measures with SRB property become the same (Appendix A). This definition of SRB measures is commonly used in different contexts.

Observation 4.1. Proposition 11.1 of $[B D V]$, shows that every map satisfying above items admits exactly two SRB measures with both basins dense and with positive Lebesgue measure.

\subsection{Example II}

The first example of a diffeomorphism with nonzero Lyapunov exponent, which is not an Anosov map, was constructed by Katok [K2]. The example was constructed up to a "surgery" on an area-preserving hyperbolic toral automorphism with two eigenvalues $0<\mu_{1}<1$ and $\mu_{2}>1$.

Starting with a hyperbolic toral endomorphism $f$ induced by the matrix $A_{n}=\left(\begin{array}{cc}n & 1 \\ 1 & 1\end{array}\right)$ using the "slow down" method near the origin, we can obtain a new map which is non uniformly hyperbolic endomorphism. The construction depends upon a real-valued "bump" function $\psi$ which is defined on the unit interval [0,1] with following properties:

1. $\psi$ is a $C^{\infty}$ function except for the origin;

2. $\psi(0)=0$ and $\psi(u)=1$ for $u \geq r_{0}$ where $0<r_{0}<1$;

3. $\dot{\psi}(u)>0$ for every $0<u<r_{0}$;

Let fix $n=3$ and denote by $\bar{\pi}: \mathbb{R}^{2} \rightarrow T^{2}$, the natural projection. Choose $p \in T^{2}$ a hyperbolic fixed point ( $d_{p} f$ has no eigenvalue of absolute value one) that $\bar{\pi}(0)=p$. We can introduce a coordinate system $\left(s_{1}, s_{2}\right)$ obtained from the eigen-directions of $A=A_{3}$ at 0 . and some $\bar{p} \in \pi^{-1}(p)$ such that $\bar{p}=\left(\ldots, a_{-1}, a_{0}=0, a_{1}, \ldots\right)$ for $a_{i} \in \mathbb{R}^{2}$. Consider the disk $D_{r}$ centered at 0 of radius $r$.

$$
D_{r}=\left\{\left(s_{1}, s_{2}\right): s_{1}^{2}+s_{2}^{2} \leq r^{2}\right\}
$$

The $f$ can be observed as the time-one map of a flow generated by following system of differential equation. 


$$
\left\{\begin{array}{l}
\dot{s_{1}}=s_{1} \log \mu_{1}, \\
\dot{s_{2}}=s_{2} \log \mu_{2} .
\end{array}\right.
$$

The answers of this one order system of equation differential considering the initial conditions $s_{1}(0)=s_{1}$ and $s_{2}(0)=s_{2}$ becomes:

$$
\left\{\begin{array}{l}
s_{1}(t)=s_{1} \mu_{1}^{t}, \\
s_{2}(t)=s_{2} \mu_{2}^{t}
\end{array}\right.
$$

Fix a sufficiently small number $r_{1}<r$ and consider the time-one map g generated by the following system of differential equations in $D_{r_{1}}$ :

$$
\left\{\begin{array}{l}
\dot{s_{1}}=s_{1} \psi\left(s_{1}^{2}+s_{2}^{2}\right) \log \mu_{1}, \\
\dot{s_{2}}=s_{2} \psi\left(s_{1}^{2}+s_{2}^{2}\right) \log \mu_{2} .
\end{array}\right.
$$

Suppose $\mu_{1}$ and $\mu_{2}$ are eigenvalues of $A$. Then by the choice of bump function we can choose a well defined $g$ of class $C^{\infty}$ in $D_{r_{1}} \backslash\{0\}$ that coincide with $f$ in some neighborhood of the boundary $\partial D_{r_{1}}$. Therefore the map

$$
G(x)= \begin{cases}f(x) & \text { if } x \in T^{2} \backslash D_{r_{1}} \\ g(x) & \text { if } x \in D_{r_{1}}\end{cases}
$$

defines an endomorphism of the Torus $T^{2}$ which is the slow down of the map $f$ at 0 .

Put $W^{u}(p)$ and $W^{s}(p)$ the global unstable-stable sets of $p$ or the projections of the eigen-lines in $\mathbb{R}^{2}$ to $T^{2}$ corresponding to the eigenvalues $\mu_{1}$ and $\mu_{2}$. Set $W=W^{u}(p) \cup W^{s}(p)$ and $X=T^{2} \backslash W$.

Letting $x=\left(s_{1}, 0\right) \in D_{r_{1}} \cap W^{s}$ and considering a vector $v \in T_{x_{0}} T^{2}$ we will have:

$$
\begin{aligned}
\lambda(x, v) & =\varlimsup_{t \rightarrow \infty} \frac{\log \left|s_{1}(t)\right|}{t} \\
& =\varlimsup_{t \rightarrow \infty}\left(\log \left|s_{1}(t)\right|\right)^{\prime}=\varlimsup_{t \rightarrow \infty} \frac{\dot{s}_{1}(t)}{s_{1}(t)} \\
& =\varlimsup_{t \rightarrow \infty}\left(\psi\left(s_{1}(t)^{2}\right) \log \mu_{1}\right),
\end{aligned}
$$

where $0<\mu_{1}<1$ and $s_{1}(t)$ is the solution of 4.3 with the initial condition $s_{1}(0)=s_{1}$. By the choice of function $\psi$ we see that $\lambda(x, v)=0$ and similarly happens to $\lambda(x, v)$ when $x \in W^{u}$. The set $W$ is a zero measure everywhere dense subset of $T^{2}$ and for $x \in X$ we 
have that Lyapunov exponents are nonzero.[BP1] In the other words, $G$ is a non- Anosov endomorphism. Such maps are also called Derived From Anosov.

The number of measures with SRB property depends on the number of the homoclinic equivalent classes referring to our main theorem.

\subsection{Example III}

This example which is somehow similar to the last one, is due to N. Sumi [S]. H shows that on the 2-torus $T^{2}$ there exists a $C^{1}$ open set $\mathrm{U}$ of $C^{1}$ maps such that every map belonging to $\mathrm{U}$ is topologically mixing but is not Anosov.

Let $f: T^{2} \rightarrow T^{2}$ be a $C^{2}$ endomorphism, and let $p \in T^{2}$ be a hyperbolic fixed point of $f$. The $p$ is a sink if all the eigenvalues of $d_{p} f$ are less than one in absolute value and $p$ is a source if all the eigenvalues of $d_{p} f$ are greater than zero in absolute value. A hyperbolic fixed point is a saddle if $p$ is neither a sink nor a source.(The same definition for periodic points.)

Suppose that $f$ has a saddle fixed point $p$. Fix $\bar{p} \in \mathbb{R}^{2}$ with $\bar{\pi}(\bar{p})=p$ where $\bar{\pi}: \mathbb{R}^{2} \rightarrow T^{2}$ and there is a lifting $\bar{f}: \mathbb{R}^{2} \rightarrow \mathbb{R}^{2}$ of $f$ such that $\bar{f}(\bar{p})=\bar{p}$. Obviously $\bar{f}$ is a diffeomorphism and $\bar{p}$ is a saddle fixed point of $\bar{f}$. For $\epsilon>0$ define $W_{\epsilon}^{s}(\bar{p}, \bar{f})=\left\{\bar{x} \in \mathbb{R}^{2}:\left|\bar{f}^{n}(\bar{x})-\bar{p}\right| \leq\right.$ $\epsilon,(n \geq 0)\}$ and $W_{\epsilon}^{u}(\bar{p}, \bar{f})=\left\{\bar{x} \in \mathbb{R}^{2}:\left|\bar{f}^{n}(\bar{x})-\bar{p}\right| \leq \epsilon, \quad(n \leq 0)\right\}$. Taking $\epsilon$ small enough then $W_{\epsilon}^{\sigma}(\bar{p}, \bar{f})$ is a $C^{1}$ curve for $\sigma=s, u$. There exists $0<\bar{\lambda}<1$ satisfying

$$
\begin{cases}\left|\bar{f}^{n}(\bar{x})-\bar{p}\right| \leq \bar{\lambda}^{n}|\bar{x}-\bar{p}| & \left(\bar{x} \in W_{\epsilon}^{s}(\bar{p}, \bar{f}), n \geq 0\right) \\ \left|\bar{f}^{-n}(\bar{x})-\bar{p}\right| \leq \bar{\lambda}^{n}|\bar{x}-\bar{p}| & \left(\bar{x} \in W_{\epsilon}^{u}(\bar{p}, \bar{f}), n \geq 0\right)\end{cases}
$$

(Hirsch and Pugh $[\mathrm{HP}])$. Put $\tilde{p}=(\ldots p p p \ldots) \in\left(T^{2}\right)^{f}$. Let Then by definition $\bar{\pi}\left(W_{\epsilon}^{\sigma}(\bar{p}, \bar{f})\right)=$ $W_{\epsilon}^{\sigma}(\tilde{p}, \bar{f})$ and $W_{\epsilon}^{\sigma}(\tilde{p}, f)$ is also a $C^{1}$ curve.

Recall the definition of $W^{\sigma}(\tilde{p}, f)$ from past and observe that stable and unstable manifolds at $\bar{p}$ of $\bar{f}$ are defined as

$$
W^{s}(\bar{p}, \bar{f})=\left\{\bar{x} \in \mathbb{R}^{2}: \lim _{n \rightarrow \infty}\left|\bar{f}^{n}(\bar{x})-\bar{p}\right|=0\right\}
$$

and

$$
W^{u}(\bar{p}, \bar{f})=\left\{\bar{x} \in \mathbb{R}^{2}: \lim _{n \rightarrow-\infty}\left|\bar{f}^{n}(\bar{x})-\bar{p}\right|=0\right\} .
$$

So we have

$$
\bar{\pi} W^{s}(\bar{p}, \bar{f})=W^{s}(\tilde{p}, f), \quad W^{u}(\tilde{p}, f)=\bar{\pi} W^{u}(\bar{p}, \bar{f})
$$


Once again let $A=A_{3}$. Let $0<\lambda<1<\mu$ the eigenvalues of $A$ and $v^{u}, v^{s}$ the eigenvectors for $\mu$ and $\lambda$ with $\left|v^{u}\right|=\left|v^{s}\right|=1$. Use the coordinates $u_{1} v^{u}+u_{2} v^{s}$ on $\mathbb{R}^{2}$ and denote by $E^{\sigma}$ the span of the eigen-vector $V^{\sigma}(\sigma=s, u)$. For $\bar{x} \in \mathbb{R}^{2}$ the tangent spaces $T_{\bar{x}} \mathbb{R}^{2}$ and $T_{\bar{\pi}(\bar{x})} T^{2}$ can be identified with $\mathbb{R}^{2}$ that means we can also use $\left(u_{1}, u_{2}\right)$-coordinates on them. Note that in this coordinate

$$
d_{\bar{x}} \bar{\pi}=\left(\begin{array}{ll}
1 & 0 \\
0 & 1
\end{array}\right): T_{\bar{x}} \mathbb{R}^{2} \longrightarrow T_{\bar{\pi}(\bar{x})} T^{2}
$$

Let $r>0$ be small enough. Then $\pi_{\left.\right|_{B_{r}(0)}}$ is injective where $B_{r}(0)$ is the ball of radius $r$ about 0 . Take a small $r$ with $0<\dot{r}<r$ and let $\delta(s)$ be a bump function that

$$
\delta(s)= \begin{cases}0 & \text { if } s \geq r \\ 1 & \text { if } s \leq r\end{cases}
$$

Now define $\bar{\phi}^{t}(\bar{x})=\left(u_{1}, e^{\delta\left(\left|\left(u_{1}, u_{2}\right)\right|\right) t} u_{2}\right)$ for $\bar{x}=\left(u_{1}, u_{2}\right) \in \mathbb{R}^{2}=E^{u} \oplus E^{s}$ and put

$$
\phi^{t}(x)= \begin{cases}\bar{\pi} \circ \bar{\phi}^{t} \circ\left(\bar{\pi}_{B_{r}(0)}\right)^{-1}(x) & \text { if } x \in \bar{\pi}\left(B_{r}(0)\right) \\ x & \text { if } x \notin \bar{\pi}\left(B_{r}(0)\right)\end{cases}
$$

Then the derivative at $p^{0}=\bar{\pi}(0)$ is $d_{p^{0}} \phi^{t}=\left(\begin{array}{cc}1 & 0 \\ 0 & e^{t}\end{array}\right)$ in the $\left(u_{1}, u_{2}\right)$-coordinates. Let $f_{A}: T^{2} \rightarrow T^{2}$ be the toral endomorphism induced by the matrix A. Since $0<\lambda<1<\mu$, $f_{A}$ is an Anosov differentiable map and $p^{0}=\bar{\pi}(0)$ is a saddle fixed point of $f_{A}$. Define $f=\phi^{\tau} \circ f_{A}$ for a fixed $\tau$ such that $\mu>e^{\tau} \lambda>1$. Note that in the $\left(u_{1}, u_{2}\right)$-coordinates the derivative of $f$ at $p^{0}$ is $d_{p^{0}} f=d_{p^{0}} \phi^{\tau} \circ d_{p^{0}} f_{A}=\left(\begin{array}{cc}1 & 0 \\ 0 & e^{\tau}\end{array}\right) \circ\left(\begin{array}{cc}\mu & 0 \\ 0 & \lambda\end{array}\right)=\left(\begin{array}{cc}\mu & 0 \\ 0 & e^{\tau} \lambda\end{array}\right)$, and thus $p^{0}$ is a source.

Let $\bar{p}^{0}=\left(\ldots, p^{0}, p^{0}, p^{0}, \ldots\right) \in\left(T^{2}\right)^{f_{A}}$. Denoting by $W_{0}^{s}\left(\tilde{p}^{0}, f_{A}\right)$ the arc-wise connected component of $W^{s}\left(\bar{p}^{0}, f_{A}\right)$ containing $\bar{p}^{0}$, then as $\bar{p}^{0}$ is a fixed point, $f\left(W_{0}^{s}\left(\bar{p}^{0}, f_{A}\right)\right)=$ $W_{0}^{s}\left(\bar{p}^{0}, f_{A}\right)$. We see that $p^{0}$ becomes a source fixed point for $f$ which is a monotonically growing function on $W_{0}^{s}\left(\bar{p}^{0}, f_{A}\right) \cap B_{r}\left(p^{0}\right)$. But the slope of the graph of $f$ on $W_{0}^{s}\left(\bar{p}^{0}, f_{A}\right)$ is less than one outside $B_{r}\left(p^{0}\right)$. Consequently the new map $f$ more than $p^{0}$ as a source, will have two new saddle fixed points $p^{1}$ and $p^{2}$ in $\bar{\pi}\left(B_{r}(0)\right)$ as well.

Proposition $\mathrm{C}$ of $[\mathrm{K} 1]$ shows that $W^{u}\left(\bar{P}^{i}\right) \cap W^{s}\left(p^{i}\right)$ becomes dense and $f$ is topologically mixing. This example by one homoclinic equivalent class, due to our main theorem, will consist of at most one measure with SRB property. 


\subsection{Example IV}

Using the similar method we can construct some non-uniformly expanding map on torus $T^{2}$. Let $f: T^{2} \rightarrow T^{2}$ be a linear expanding map. Linear expanding maps are topologically exact(for any $x \in M$, and any $\epsilon>0$, then exist some $n \in \mathbb{N}$ that $f^{n}\left(B_{\epsilon}(x)\right)=$ $M)$. Fix some covering $\mathcal{U}$ by domains of injectivity for $f$ and some $U \in \mathcal{U}$ containing a fixed (or periodic) point $p$. Deforming $f$ by a pitchfork bifurcation on $U$ in a way that $p$ becomes a saddle for the perturbed local diffeomorphism $g$. In particular, we can do it in the $C^{2}$-topology and that $g$ coincides with $f$ in the $U^{c}$ where uniform expansion holds. We take the deformation in a way that $f$ is not too contracting in $U$. This can guarantee that $f$ still becomes topologically exact. $[\mathrm{CV}]$ So that by Theorem $\mathrm{B}$ there would exist at most one measure with SRB property for that.

For more details and examples of this type we refer to $[\mathrm{BDV}],[\mathrm{LP}],[\mathrm{HG}],[\mathrm{V}]$. 


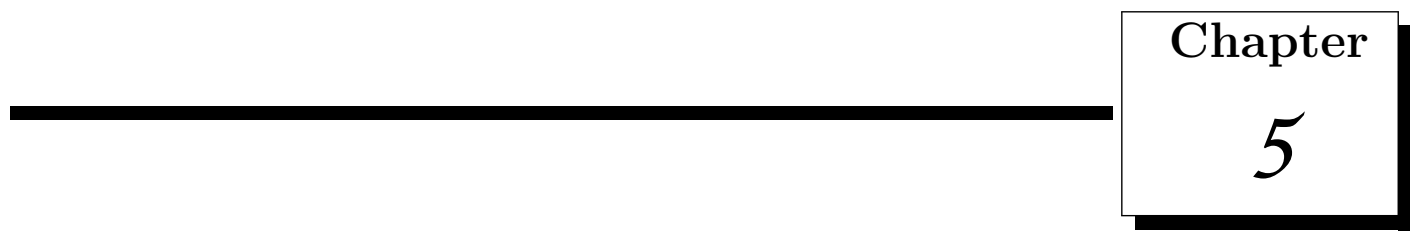

Appendix A

In the last step of the proof of Katok Closing lemma for $C^{2}$ endomorphisms (local diffeomorphisms), we need to show that $z$ is a hyperbolic periodic point. For $0<\gamma<1$, from proposition 2.2, let consider the following two cones as:

$$
\begin{aligned}
& C_{\gamma}^{u}=\left\{\left(w_{1}, w_{2}\right) \in \mathbb{R} \times \mathbb{R}:\left\|w_{1}\right\| \leq \gamma\left\|w_{2}\right\|\right\} ; \\
& C_{\gamma}^{s}=\left\{\left(w_{1}, w_{2}\right) \in \mathbb{R} \times \mathbb{R}:\left\|w_{2}\right\| \leq \gamma\left\|w_{1}\right\|\right\} .
\end{aligned}
$$

On the sake of simplicity let us take $m=1$. For $\tilde{x} \in \tilde{\Delta}_{l},(u, v) \in B_{\eta_{l}} \times B_{\eta_{l}}$ and $\gamma=\frac{1-\lambda}{20}$, let $w=\left(w_{1}, w_{2}\right) \in C_{\gamma}^{u}$. Obtaining the hyperbolicity of $z$ we need to show the following three items:

- Exist expanding and contracting sub-spaces $E^{u}(\bar{z}), E^{s}(\bar{z})$ that $T_{z} M=E^{u}(\bar{z}) \oplus E^{s}(\bar{z})$;

- $\left(d F_{\bar{z}}\right) \tilde{C}_{\gamma}^{u} \subset \tilde{C}_{\hat{\gamma}}^{u}, \quad \& \quad\left(d F_{\bar{z}}^{-1}\right) \tilde{C}_{\gamma}^{s} \subset \tilde{C}_{\hat{\gamma}}^{s}$

- $\|\left(d F_{\bar{z}}(w)\left\|_{\tilde{x}}^{\prime}>\hat{C}\right\| w\left\|_{\tilde{x}}^{\prime}, \quad \& \quad\right\|\left(d F_{\bar{z}}^{-1}\right)(w)\left\|_{\tilde{x}}^{\prime}>(\hat{C})\right\| w \|\right.$.

Where $\tilde{C}_{\gamma}^{u}=\left(\Phi_{\tilde{x}}\right)_{(u, v)}\left(C_{\gamma}^{u}\right), \tilde{C}_{\gamma}^{s}=\left(\Phi_{\tilde{x}}\right)_{(u, v)}\left(C_{\gamma}^{s}\right)$ and $\gamma>\hat{\gamma}>0, \hat{C}>1$. First we check item 3 and 2 then we see item 1. By definition for $w=\left(w_{1}, w_{2}\right) \in C_{\gamma}^{u}$ then 
$\|w\| \leq(1-\gamma)^{-1}\left\|w_{2}\right\|$ and using proposition 2.1:

$$
\begin{aligned}
\left\|\left(d F_{\tilde{x}}\right)_{(u, v)}\left(w_{1}, w_{2}\right)\right\| & =\left\|\left(A_{\tilde{x}} w_{1}+B_{\tilde{x}} w_{2}\right)+\left(d h_{\tilde{x}}\right)_{(u, v)}\left(w_{1}, w_{2}\right)\right\| \\
& \geq\left\|B_{\tilde{x}} w_{2}\right\|-\left\|A_{\tilde{x}}\right\|-\left\|\left(d h_{\tilde{x}}\right)_{(u, v)}\right\|\|w\| \\
& \geq \lambda^{-1}\left\|w_{2}\right\|-\lambda\left\|w_{1}\right\|-\frac{(1-\lambda)^{2}}{100}\|w\| \\
& \geq \lambda^{-1}(1-\gamma)\|w\|-\lambda \gamma\|w\|-\frac{(1-\lambda)^{2}}{100}\|w\| \\
& =\left(\lambda^{-1}-\left(\frac{\lambda^{-1}(1-\lambda)}{20}+\frac{\lambda(1-\lambda)}{20}+\frac{(1-\lambda)^{2}}{100}\right)\right)\|w\| \\
& >\left(\frac{1}{2}+\frac{1}{2 \lambda}\right)\|w\| .
\end{aligned}
$$

By 2.7 we see that above inequality becomes valid. (Figure 5.1)

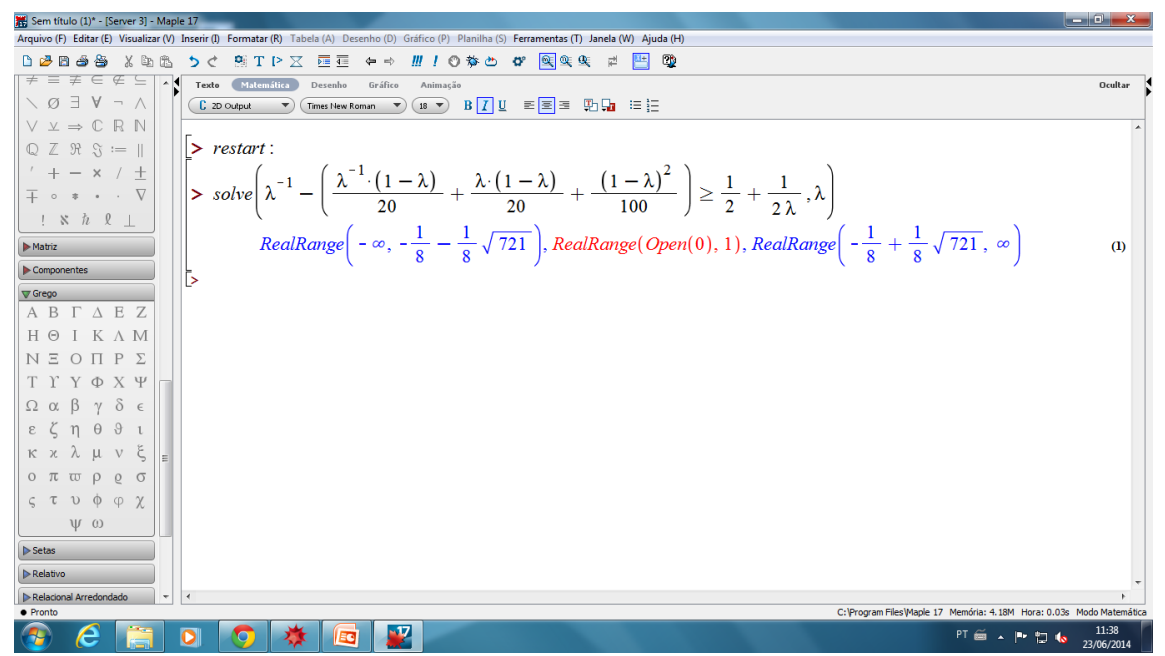

Figure 5.1

Similarly happens that

$$
\left\|\left(d F_{\tilde{x}}^{-1}\right)_{(u, v)}\left(w_{1}, w_{2}\right)\right\|>\left(\frac{1}{2}+\frac{1}{2 \lambda}\right)\|w\|
$$

From proof of the first item of proposition 2.2 referred to Katok's paper [K1], we see that for $\lambda$ as 2.7:

$$
\left(d F_{\tilde{x}}\right)_{(u, v)} C_{\gamma}^{u} \subset C_{\lambda \gamma}^{u}, \quad \& \quad\left(d F_{\tilde{x}}^{-1}\right)_{(u, v)} C_{\gamma}^{s} \subset C_{\lambda \gamma}^{s}
$$


Now let consider the periodic point $z$, which here, we have assumed it to be a fixed point. Put $z=\Phi_{\tilde{x}}(u, v)$, then from proposition 2.1,

$$
\begin{aligned}
d F_{\bar{z}} & =\left(d \Phi_{\tilde{f}(\tilde{x})}\right)_{(u, v)} \circ\left(d F_{\tilde{x}}\right)_{(u, v)} \circ\left(d \Phi_{\tilde{x}}^{-1}\right)_{z} \\
& =\left(d \Phi_{\tilde{f}(\tilde{x})}\right)_{(u, v)} \circ\left(d \Phi_{\tilde{x}}^{-1}\right)_{z} \circ\left(d \Phi_{\tilde{x}}\right)_{(u, v)} \circ\left(d F_{\tilde{x}}\right)_{(u, v)} \circ\left(d \Phi_{\tilde{x}}^{-1}\right)_{z} .
\end{aligned}
$$

Observe that in above equation, $\left(d \Phi_{\tilde{x}}\right)_{(u, v)} \circ\left(d F_{\tilde{x}}\right)_{(u, v)} \circ\left(d \Phi_{\tilde{x}}^{-1}\right)_{z}$ is an operator from $T_{z} M \rightarrow$ $T_{z} M$ and $\left(d \Phi_{\tilde{f}(\tilde{x})}\right)_{(u, v)} \circ\left(d \Phi_{\tilde{x}}^{-1}\right)_{z}$ transforms the $\|\cdot\|_{\tilde{x}}^{\prime}$ norm into the $\|\cdot\|_{\tilde{f}(\tilde{x})}^{\prime}$.

Since $d \Phi_{\tilde{x}}$ is transforming the Euclidean norm from $\mathbb{R}^{2}$ to the norm $\|\cdot\|_{\tilde{x}}^{\prime}$, so the properties 5.3 happens to $\tilde{C}_{\gamma}^{u}$ and $\tilde{C}_{\gamma}^{s}$ :

$$
\left(d F_{\bar{z}}\right) \tilde{C}_{\gamma}^{u} \subset \tilde{C}_{\hat{\lambda} \gamma}^{u} \quad \& \quad\left(d F_{\bar{z}}^{-1}\right) \tilde{C}_{\gamma}^{s} \subset \tilde{C}_{\hat{\lambda} \gamma}^{s}
$$

for some $\hat{\lambda}<1$ which can be obtained by choosing a suitable small enough $\varrho$ in 2.15 .

From 5.1 and 5.2 and taking $\tau$ in 2.16 equal with

$$
\tau=\frac{1+\frac{1-\lambda}{2}}{1+\frac{1-\lambda}{2 \lambda}}
$$

then we see that for $w \in \tilde{C}_{\gamma}^{u}$

$$
\left\|\left(d F_{\bar{z}}\right)(w)\right\|_{\tilde{f}(\tilde{x})}^{\prime}>\left(1+\frac{1}{2}+\frac{1}{2 \lambda}\right)\|w\|_{\tilde{x}}^{\prime} \Rightarrow^{5.7}\left\|\left(d F_{\bar{z}}\right)(w)\right\|_{\tilde{x}}^{\prime}>\left(1+\frac{1-\lambda}{2}\right)\|w\|_{\tilde{x}}^{\prime} .
$$

and similarly for $w \in \tilde{C}_{\gamma}^{s}$,

$$
\left\|\left(d F_{\bar{z}}^{-1}\right)(w)\right\|_{\tilde{x}}^{\prime}>\left(1+\frac{1-\lambda}{2}\right)\|w\|_{\tilde{x}}^{\prime}
$$

We can define respectively the following expanding and contracting sub-spaces:

$$
E^{u}(\bar{z}):=\bigcap_{k=0}^{\infty} d F_{\bar{z}}^{k}\left(\tilde{C}_{\gamma}^{u}\right), \quad \& \quad E^{s}(\bar{z}):=\bigcap_{k=0}^{\infty} d F_{\bar{z}}^{-k}\left(\tilde{C}_{\gamma}^{s}\right),
$$

that $E^{s}(\bar{z}) \oplus E^{u}(\bar{z})=\{0\}$, and so $T_{z} M=E^{s}(\bar{z}) \oplus E^{u}(\bar{z})$.

Following proposition is a corollary of proposition 2.2 .

Proposition 5.1. Suppose that $\tilde{x}, f_{\tilde{x}}^{m}(x) \in \tilde{\Delta}_{1}$ Then for $\delta \leq h \eta(l) / 2, N \in U_{\tilde{x}}^{\gamma(\chi), \delta, h}$ we have:

- $f_{\tilde{f}^{m} \tilde{x}}^{-m}(N) \cap R(\tilde{x}, h) \in U_{\tilde{x}}^{\lambda^{-m} \gamma(\chi), \delta\left(\frac{1+\lambda}{2}\right)^{-m}, h} ;$ 
- for any two points $y_{1}, y_{2} \in N \in U_{\tilde{f}^{m}(\tilde{x})}^{\gamma(\chi), \delta, h}$;

$$
d_{\tilde{x}}^{\prime}\left(f_{\tilde{f}^{m}(\tilde{x})}^{-m}\left(y_{1}\right), f_{\tilde{f}^{m}(\tilde{x})}^{-m}\left(y_{2}\right)\right)<(1 / 2+1 / 2 \lambda)^{-m} d_{\tilde{f}^{m}(\tilde{x})}^{\prime}\left(y_{1}, y_{2}\right)
$$

where $\|.\|_{\tilde{x}}^{\prime}$ is the Lyapunov norm as defined in chapter 2.

Proof. Considering the proposition 2.2, for $\tilde{x} \in \tilde{\Delta}_{1}, \delta \leq h \eta_{l} / 2$,

$$
N \in U_{\tilde{x}}^{\gamma, \delta, h} \Rightarrow f(N) \cap R(\tilde{f}(\tilde{x}), h) \in U_{\tilde{f}(\tilde{x})}^{\lambda \gamma,\left(\frac{1+\lambda}{2}\right), h} .
$$

We can conclude that $f_{\tilde{f}^{m}(\tilde{x})}^{-1}(N) \in U_{\tilde{x}}^{\lambda^{-1} \gamma, \delta\left(\frac{1+\lambda}{2}\right)^{-1}, h}$. 


\section{Bibliography}

[A] Martin. Andersson, Robust ergodic properties in partially hyperbolic dynamics. (English summary) Trans. Amer. Math. Soc. 362, (2010), no. 4, 1831-1867.

[BDV] Christian Bonatti; Lorenzo J Díaz; Marcelo Viana, Dynamics beyond uniform hyperbolicity. A global geometric and probabilistic perspective. Encyclopaedia of Mathematical Sciences, 102. Mathematical Physics, III. Springer-Verlag, Berlin, 2005.

[BP1] Luis Barreira; Yakov B. Pesin, Lyapunov exponents and smooth ergodic theory. University Lecture Series, 23. American Mathematical Society, Providence, RI, 2002.

[BP2] L. Barreira; Y. Pesin, Nonuniform hyperbolicity. Dynamics of systems with nonzero Lyapunov exponents. Encyclopedia of Mathematics and its Applications, 115. Cambridge University Press, Cambridge, 2007.

[Ch] Yong Moo, Chung, Shadowing property of non-invertible maps with hyperbolic measures. (English summary) Tokyo J. Math. 22, (1999), no. 1, 145-166.

[CV] A.Castro; P.Varandas, Equilibrium states for non-uniformly expanding maps: decay of correlations and strong stability. (English summary) Ann. Inst. H. Poincar Anal. Non Linaire 30, (2013), no. 2, 225-249.

[HG] Baolin, He; Shaobo Gan; Robustly non-hyperbolic transitive endomorphisms on $T^{2}$. Proc. Amer. Math. Soc. 141 (2013), no. 7, 2453-2465.

[HHTU] F. Rodriguez Hertz; M. Rodriguez Hertz; A. Tahzibi; R. Ures, Uniqueness of SRB measures for transitive diffeomorphisms on surfaces. Comm. Math. Phys. 306 (2011), no. 1, 35-49.

[HP] M. Hirsch and C. Pugh, Stable manifolds and hyperbolic sets, Global Analysis, Proc. Sympos. Pure Math., 14, Amer. Math. Soc. (1970), 133-163. 
[HPSh] M.W. Hirsch, C. C. Pugh, M. Shub, Invariant manifolds. Lecture Notes in Mathematics, Vol. 583. Springer-Verlag, Berlin-New York, 1977.

$[\mathrm{KH}]$ Anatole Katok; Boris Hasselblatt; Introduction to the modern theory of dynamical systems. (English summary) With a supplementary chapter by Katok and Leonardo Mendoza. Encyclopedia of Mathematics and its Applications, 54. Cambridge University Press, Cambridge, 1995.

[K1] A. Katok, Lyapunov exponents, entropy and periodic orbits for diffeomorphisms. Inst. Hautes Études Sci. Publ. Math., No. 51 (1980), 137-173.

[K2] A. Katok, Bernoulli diffeomorphism on surface, Ann. of Math (2)110, (1979), 529547.

[K3] Ittai Kan, Open sets of diffeomorphisms having two attractors each with an everywhere dense basin, Bull.Amer. Math. Soc 31, (1994), no. 1, 68-74.

[LP] Lizana, Cristina; Pujals, Enrique, Robust transitivity for endomorphisms. Ergodic Theory Dynam. Systems 33 (2013), no. 4, 1082-1114.

[LQ] P.-D. Liu, M. Qian, Smooth Ergodic Theory of Random Dynamical Systems, Lect. Notes Math., Vol. 1606,Springer-Verlag, New York, 1995.

[LS] F. Ledrappier and J.-M. Strelcyn, A proof of the estimation from below in Pesin entropy formula,Ergod. Th. \& Dynam. Sys. 2, (1982), 203-219.

[LY] F. Ledrappier and L.-S. Young, The metric entropy of diffeomorphisms, Ann. Math.122, (1985), 509-574.

[M4] Eugen. Mihailescu, Equilibrium measures, prehistories distributions and fractal dimensions for endomorphisms. Discrete Contin. Dyn. Syst. 32 (2012), no. 7, 2485-2502.

[M2] P. Mattila, Geometry of sets and measures in euclidean spaces. Cambridge Studies in Advanced Mathematics, 44. Cambridge University Press, Cambridge, 1995.

[M3] John W. Milnor, Attractors, Scholarpedia,(2006).

[OV] K. Oliveira, M. Viana, Teoria Ergdica Um Curso Introdutrio - Impa,2007.

[P] Feliks Przytycki; Anosov endomorphisms. Studia Math. 58 (1976), no. 3, 249-285.

[QXZ] Min, Qian ;Jian-sheng, Xie; Shu, Zhu Smooth ergodic theory for endomorphisms. Lecture notes in mathematics, Vol. 1978 . Springer-Verlag, Berlin Heidelberg, 2009. 
[QZ] Min Qian; Shu Zhu, SRB measures and Pesin's entropy formula for endomorphisms. (English summary) Trans. Amer. Math. Soc.,354 (2002), no. 4, 1453-1471.

[R1] V. A. Rokhlin, On the fundamental ideas of measure theory, Transl. Amer. Math. Soc, Series 1, 10 (1962), 1-52.

[R2] D. Ruelle, Elements of differentiable dynamics and bifurcation theory. Academic Press, Inc., Boston, MA, 1989.

[RY] Aaditya Rangan; Lai-Sang Young, Dynamics of spiking neurons: between homogeneity and synchrony. (English summary) J. Comput. Neurosci., 34 (2013), no. 3, 433-460.

[Sh] Michael Ira Shub; Endomorphisms of compact differentiable manifolds. Thesis (Ph.D.) University of California, Berkeley. 1967. 41 pp.

[S] Naoya Sumi, A class of diferentiable toral maps which are topologically mixing, Proc. Amer. Math. Soc. 127 (1999), 915-924.

[T1] Masato Tsujii, Physical measures for partially hyperbolic surface endomorphisms, Acta Math. 194 (2005), no. 1, 37-132.

[UW] M. Urbanski; Ch. Wolf, SRB measures for Axiom A endomorphisms. Math. Res. Lett. 11 (2004), no. 5-6, $785 ? 797$.

[V] M. Viana, Multidimensional nonhyperbolic attractors, Inst. Hautes tudes Sci. Publ. Math., 85 (1997), 63-96. 335, (2002), 1057-1062.

[W] P. Walters, An introduction to ergodic theory. Graduate Texts in Mathematics, 79. Springer-Verlag, New York-Berlin, 1982. 